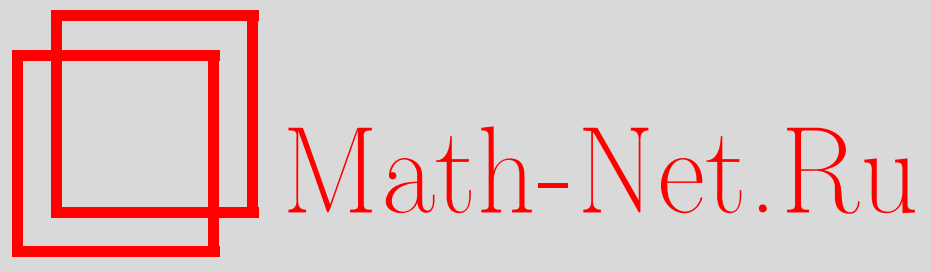

В. А. Садовничий, В. Е. Подольский, Следы операторов, УМН, 2006, том 61, выпуск 5, 89-156

DOI: https://doi.org/10.4213/rm4833

Использование Общероссийского математического портала Math-Net.Ru подразумевает, что вы прочитали и согласны с пользовательским соглашением http://www . mathnet.ru/rus/agreement

Параметры загрузки:

IP : 54.197 .217 .227

26 апреля 2023 г., 07:10:28

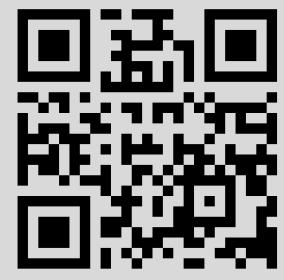


УДК 517.984

\section{Следы операторов}

\section{В. А. Садовничий, В. Е. Подольский}

Настоящий обзор посвящен истории и современному состоянию теории регуляризованных следов линейных операторов. Основное внимание уделено операторам с дискретным спектром. Несколько дополнений посвящены некоторым смежным разделам спектральной теории и постановке ряда важных нерешенных задач.

Библиография: 183 названия.

\section{СоДЕРЖАНИЕ}

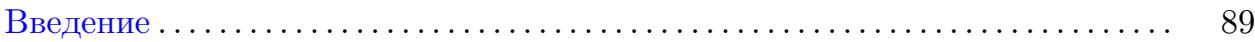

Регуляризованные следы. Обыкновенные дифференциальные операторы. 96

Регуляризованные следы. Общая теория ..................... 117

Дополнение 1. Функция спектрального сдвига и формула Крейна . . . . . . 132

Дополнение 2. $\zeta$-функция, $\theta$-функция, некоммутативный вычет и спект-

ральная асимметрия ............................... 137

Дополнение 3. Теория индекса линейных операторов ................ 140

Дополнение 4. О приближенном вычислении первых собственных чисел . 141

Дополнение 5. Некоторые другие результаты .................... 144

Дополнение 6. Некоторые нерешенные задачи...................... 146

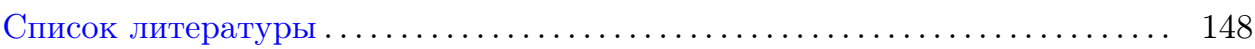

\section{Введение}

Теория следов линейных операторов начинается с фундаментального результата линейной алгебры: матричный след линейного оператора инвариантен относительно выбора базиса и совпадает со спектральным следом.

Роль этого инварианта и его следствий в различных разделах алгебры, анализа, геометрии очень большая. Наша цель - дать обзор той части современной теории операторов в гильбертовом пространстве, которая была рождена в поиске аналогов инвариантного следа для операторов, заведомо не имеющих следа в обычном смысле. 
Однако, вначале конечномерный результат был перенесен на случай бесконечномерных операторов со следом - так называемых ядерных операторов ${ }^{1}$, а именно, было доказано (см. [1]), что если оператор $A$ - ядерный, то для любой пары $\left(\left\{\varphi_{n}\right\}_{n=1}^{+\infty},\left\{\psi_{n}\right\}_{n=1}^{+\infty}\right)$ ортонормированных базисов верно

$$
\sum_{n=1}^{+\infty}\left(A \varphi_{n}, \varphi_{n}\right)=\sum_{n=1}^{+\infty}\left(A \psi_{n}, \psi_{n}\right)
$$

и также верно равенство, известное как теорема В. Б. Лидского [2],

$$
\sum_{n=1}^{+\infty}\left(A \varphi_{n}, \varphi_{n}\right)=\sum_{n} \lambda_{n}
$$

где $\left\{\lambda_{n}\right\}$ - все собственные числа оператора $A$ (если $A$ не имеет собственных чисел, то в правой части (2) стоит 0). Этими результатами классическая теория была завершена, так как здесь в максимальной общности охвачен весь класс операторов со следом, и с этого места начинается теория регуляризованных следов линейных операторов.

По-видимому, первые результаты, положившие начало теории регуляризованных следов операторов, получил в 1947-52 годах И. М. Лифшиц (завершающая работа цикла [3], там можно найти дальнейшие ссылки), рассмотревший задачу о вычислении следа оператора $F(L+\Lambda)-F(L)$, где $L-$ невозмущенный эрмитов оператор, $\Lambda$ - конечномерный оператор возмущения, а $F(x)$ - некоторая (принадлежащая достаточно широкому классу) функция. Оказалось, формулы регуляризованных следов имели физический смысл: И. М. Лифшиц с помощью своей формулы посчитал изменение свободной энергии кристалла при внедрении в него чужеродной примеси. Смысл регуляризованного следа как меры дефекта полной энергии системы при ее возмущении в ситуации, когда сама полная энергия системы (точнее, рассматриваемой модели) бесконечна, является одним из важнейших критериев при оценке содержания полученных теорией результатов, хотя и далеко не единственным.

Первые результаты И. М. Лифшица относились к случаю оператора $L$ со спектром произвольной природы и были им применены к оператору с непрерывным спектром. Замечательное развитие этих результатов было сделано М. Г. Крейном [4], в дальнейшем этот раздел теории регуляризованных следов успешно развивался, ему и его приложениям было посвящено несколько обзоров и разделов монографий (см., например, [5], [6]), и мы не видим необходимости вновь подробно рассматривать это направление, ограничившись обзором в дополнении 1 наиболее важных работ и тех аспектов этой теории, которые не отражены в имеющейся литературе.

\footnotetext{
${ }^{1} s$-числами компактного оператора $A$ называются упорядоченные по убыванию собственные числа положительного оператора $\sqrt{A A^{*}}$. Множество компактных операторов, $s$-числа которых образуют сходящийся ряд $\sum s_{n}^{p}$ при некотором $p>0$, традиционно обозначается $\mathfrak{S}_{p}$; при $p \geqslant 1$ это множество образует симметрично-нормированный идеал в алгебре всех ограниченных операторов с нормой $\|\cdot\|_{p}=\left(\sum s_{n}^{p}\right)^{1 / p}$. При $p=1$ эта норма называется ядерной нормой операторов, при $p=2$ - нормой Гильберта-Шмидта или абсолютной нормой. Подробное изложение теории симметрично-нормированных идеалов см. в [1].
} 
Основной нашей целью будет история развития теории регуляризованных следов дискретных ${ }^{2}$ операторов и той части теории для операторов с непрерывным спектром, которая по идеологии и техническому аппарату ближе теории для дискретных операторов.

Началом этого раздела теории регуляризованных следов операторов стала работа И. М. Гельфанда и Б. М. Левитана [7], в которой для оператора ШтурмаЛиувилля

$$
-y^{\prime \prime}+q(x) y=\lambda y, \quad y^{\prime}(0)=0, \quad y^{\prime}(\pi)=0,
$$

$q(x) \in C^{1}[0, \pi]$, при условии ${ }^{3} \int_{0}^{\pi} q(x) d x=0$ была получена формула

$$
\sum_{n=0}^{\infty}\left(\mu_{n}-\lambda_{n}\right)=\frac{1}{4}(q(0)+q(\pi)),
$$

здесь $\mu_{n}$ - собственные числа оператора (3), а $\lambda_{n}=n^{2}-$ собственные числа такого же оператора с $q(x) \equiv 0$.

Эта формула породила большую и очень важную теорию, начавшуюся с исследования конкретных операторов и в дальнейшем охватившую изучение регуляризованных следов дискретных операторов в общем виде.

Для дискретных операторов на первый взгляд естественна (в соответствии с общими идеями регуляризации расходящихся рядов) следующая постановка задачи обобщения понятия следа: при расходимости ряда из матричных элементов оператора доказать как аналог формулы (1) соотношение

$$
\sum_{n=1}^{+\infty}\left(\left(A \varphi_{n}, \varphi_{n}\right)-\left(A \psi_{n}, \psi_{n}\right)\right)=0
$$

однако легко заметить, что без существенных уточнений в постановке вопроса формулы (1) и (5) равносильны. Это следует из простого факта теории числовых рядов: если ряд из матричных элементов расходится в каком-то базисе $\left\{\varphi_{n}\right\}$, то существует такая перенумерация векторов этого базиса, которую можно принять за другой базис $\left\{\psi_{n}\right\}$, что ряд (5) расходится. Отсюда следует, что для любых неядерных операторов $A$ равенство (5) не может быть верно для любых пар базисов. Таким образом, разумная постановка основной задачи принимает вид: указать класс операторов $A$ и соответствующий класс пар базисов $\left(\left\{\varphi_{n}\right\},\left\{\psi_{n}\right\}\right)$, для которых верна инвариантность следа в смысле (5).

Эта постановка задачи выдвигает на первый план следующую: выбор базисов - хотя ясно, что второй из этих базисов должен быть в каком-то смысле близок к первому, но выбор первого базиса для разных классов операторов неочевиден, и для содержательной постановки задачи этот выбор базиса должен быть обусловлен вескими внутренними причинами.

Для дискретных операторов естественной идеей, направлявшей выбор базисов, являлась спектральная формулировка следа (2): в качестве одного из

\footnotetext{
2 Дискретным называется оператор, имеющий компактную резольвенту.

${ }^{3}$ Условие равенства нулю среднего от потенциала не является ограничительным, так как всегда может быть достигнуто сдвигом спектра на константу.
} 
базисов выбирается базис из собственных векторов оператора $A$-разумеется, в предположении, что он есть, а для определения второго базиса оператор "расщепляется" в сумму двух ${ }^{4}: A=A_{0}+B$, причем предполагается подчиненность оператора $B$ оператору $A_{0}$, и формула (5) приобретает вид

$$
\sum_{n=1}^{+\infty}\left(\left(A \varphi_{n}, \varphi_{n}\right)-\left(A \psi_{n}, \psi_{n}\right)\right)=\sum_{n=1}^{+\infty}\left(\lambda_{n}-\mu_{n}+\left(B \varphi_{n}, \varphi_{n}\right)\right)=0
$$

где $\left\{\varphi_{n}\right\}$ - базис из собственных векторов оператора $A_{0}$ с собственными числами $\left\{\lambda_{n}\right\},\left\{\psi_{n}\right\}$ - базис из собственных векторов оператора $A$ с собственными числами $\left\{\mu_{n}\right\}$, а степень подчиненности оператора $B$ оператору $A_{0}$ по сути является мерой близости базисов.

Укажем здесь на важное, часто встречающееся в приложениях обстоятельство - в общем положении мы вынуждены рассматривать суммирование в (6) со скобками, так как если оператор $A_{0}$ имеет кратное собственное число, то в соответствующем инвариантном подпространстве у нас нет приоритетного выбора базиса и вновь простая перенумерация векторов может привести нас к потере смысла задачи, причем в силу симметрии основной формулы (5) мы должны рассматривать как единое целое след конечномерной части оператора, действующей в сумме подпространств, отвечающих пусть различным, но близким (в смысле данного возмущения: мы должны учитывать, что в рассматриваемом классе возмущений существует такое, что при нем эта группа собственных чисел сольется в одно высокократное) собственным числам ${ }^{5}$.

Итак, первая основная задача теории регуляризованных следов - установить пределы справедливости обобщенной инвариантности следа, представленной формулой (6).

Вторая основная задача теории регуляризованных следов - для случаев неинвариантности следа в смысле (6) определить меру этой неинвариантности, найдя дальнейшие поправки к общему члену ряда (6), превращающие его в сходящийся ряд. Дело в том, что величина $\left(B \varphi_{n}, \varphi_{n}\right)$ хорошо известна еще из конечномерной теории возмущений дискретного спектра - это первая поправка теории возмущений для изолированного собственного числа. Поэтому, на формулу (6) можно смотреть следующим образом: среднее отклонение собственных чисел при возмущении "равно" сумме первых поправок. Если операторы таковы, что формула (6) не имеет места, то возникает следующая задача: можно ли конечным числом поправок, и каким минимально, достичь аналогичного эффекта?

Третья основная задача теории регуляризованных следов - для конкретных операторов математической физики (обычно это дифференциальные, псевдодифференциальные и интегральные операторы $\left.{ }^{6}\right)$ выделить из регуляризатора "точную" расходящуюся часть, выраженную в терминах собственных чисел невозмущенного оператора, и суммировать сходящийся остаток к конечному выражению, содержащему только функционалы от операторов $A_{0}$ и $B$. Как

4“. . . производится разделение оператора . . . на две части, одна из которых должна быть простой, а другая малой" (П.А. М. Дирак. "Принципы квантовой механики").

${ }^{5}$ Мы более не будем мотивировать необходимость и способы расстановки скобок, ограничиваясь описанием получающейся расстановки в конкретных задачах.

${ }^{6}$ в дальнейшем ДО, ПДО и ИО. 
правило, полученный ответ (также называемый регуляризованным следом) содержит нетривиальную информацию о возмущенной системе.

В исторической ретроспективе отметим, что в начале развития теории в основном решалась именно третья из сформулированных задач: содержательный раздел математической физики и должен стартовать с изучения важных в приложениях операторов. При этом разработанные методы "автоматически" давали разложение регуляризатора на сходящееся и расходящееся слагаемые. То, что при этом решалась и первая задача, часто оставалось вне поля зрения авторов, частичным исключением являются работа Л. А. Дикого [8], несколько основанных на ней работ К. Хальберга, В. Крамера и Р. Гилберта (см. [9] и там дальнейшие ссылки) и работа М. Г. Гасымова [10].

Для теории регуляризованных следов операторов со спектром произвольной природы аналогом постановки задачи (5) явилась задача о вычислении следа операторного интеграла Стилтьеса по мере, являющейся разностью спектральных семейств двух различных операторов. Так, в своих работах И. М. Лифшиц исследовал формальное равенство

$$
\operatorname{Tr}(F(L+\Lambda)-F(L))=\operatorname{Tr} \int F(\lambda) d\left(E_{\lambda}^{\prime}-E_{\lambda}\right)=\int F(\lambda) d \operatorname{Tr}\left(E_{\lambda}^{\prime}-E_{\lambda}\right),
$$

где символами $E_{\lambda}$ и $E_{\lambda}^{\prime}$ обозначены соответственно разложения единиц операторов $L$ и $L+\Lambda$. С естественной корректировкой все три задачи теории сохраняют свой смысл и в этом случае.

С нашей точки зрения, историю теории регуляризованных следов можно в настоящий момент разделить на два периода развития, в соответствии с которыми мы и разбили основной текст работы на две части, а уже в рамках этих частей мы старались объединять обсуждаемые работы по близости задач, методов и используемой технике.

Первый период: с начала 1950-х годов и до конца 1970-х. В это время на примере простейших операторов, в первую очередь Штурма-Лиувилля, были предложены основные постановки и многочисленные идеи и методы решения задач, сформировались центральные направления исследований.

Начиная с середины 1960-х годов, в первую очередь с появлением в работе В. Б. Лидского и В. А. Садовничего [11] первого в теории общего метода нахождения регуляризованных следов широкого класса обыкновенных ДО, а также многих задач со сложным вхождением спектрального параметра, этот период находился в фазе бурного развития. Метод Лидского-Садовничего обладает вычислительной законченностью, и в теории следов дискретных операторов он надолго захватил лидирующую роль, немало работ по его развитию и применению продолжает выходить и в настоящее время, и мы включили обзор таких работ в первую часть.

Также начиная с середины 1960-х годов, после выхода работы Л. Д. Фаддеева [12] и особенно работы В. С. Буслаева и Л. Д. Фаддеева [13] бурно развивалась и теория регуляризованных следов ДО, возникающих в теории рассеяния и имеющих непрерывный спектр.

В эти же временные рамки попал и большой цикл работ, связанный с развитием метода обратной задачи решения некоторых классических нелинейных 
эволюционных уравнений. После того, как в работе В. Е. Захарова и Л. Д. Фаддеева [14] на примере уравнения Кортевега-де Фриза было показано, что формулы следов некоторых возникающих в этом методе операторов интерпретируются как первые интегралы соответствующих рассматриваемым эволюционным уравнениям бесконечномерных гамильтоновых систем, почти не было работы по этой тематике, в которой бы не находились формулы следов. Ввиду необозримости этих результатов мы, за редким исключением, ограничимся ссылками на монографии, в которых эта тема обсуждается подробно и имеется достаточная библиография. Несколько предметнее в рамках этого направления мы остановимся только на периодическом случае, так как здесь формулы следов выступали и в качестве весьма своеобразного метода восстановления потенциала.

Второй период: с конца 1970-х и по настоящее время. После описанного выше периода бурного развития и получения большого количества результатов в рамках эксплуатации имевшихся общих методов, вместе с сужением круга стандартно решаемых задач постепенно в теории на первый план стали выходить задачи для дискретных ДО в частных производных, в решении которых (за исключением задач с разделяющимися переменными) до этого не было никаких продвижений. Принципиальным стал поворот теории к изучению следов абстрактных операторов и приложение этих результатов к конкретным операторам математической физики. Можно сказать, что водоразделом стал переход от третьей задачи теории следов (мы имеем в виду данную выше классификацию) к первой и второй. Работой, фактически открывшей данное направление, стала статья В. А. Садовничего и В. В. Дубровского [15], роль которой особенно ясна в контексте почти одновременно вышедшей работы тех же авторов [16], в которой впервые был найден регуляризованный след дискретного оператора, задаваемого дифференциальным выражением в частных производных. K настоящему времени теория следов абстрактных операторов ощутимо продвинулась вперед, дойдя в нашей работе [17] до формулировок, в определенном смысле "зеркальных" теореме Лидского.

Также в этот период были получены формулы регуляризованных следов во многих "штучных" задачах, к которым либо вообще не удается применить существующие общие методы, либо индивидуальный подход привел к существенно более сильным результатам по сравнению с общими теоремами. Мы выделим здесь работы В.А. Садовничего и В. В. Дубровского [18] и В. Е. Подольского [19], [20] по следам для оператора Лапласа-Бельтрами на сферах и проективных пространствах, оригинальную идею нового метода получения формул следов П. Лакса [21], цикл работ Б. Саймона с сотрудниками (см. работу [22] и имеющиеся там дальнейшие ссылки) по получению формул следов в непериодическом случае, структурно сходных с упоминавшимися формулами периодической задачи, работы В. А. Любишкина и В. Е. Подольского [23], В. Е. Подольского [19], Б. Саймона и др. [24], в которых с различных точек зрения развивается метод суммирования по Абелю регуляризованных следов.

Как мы уже говорили, ряд разделов теории, а также ряд примыкающих вопросов мы по различным причинам не внесли в основной текст, посвятив им несколько дополнений. 
Дополнение 1 посвящено формуле следа типа Крейна и теории функции спектрального сдвига. Как мы уже отмечали, именно этот раздел теории следов нашел хорошее освещение в ряде обзоров и монографий, кроме того, он достаточно замкнут и независим от остальной теории, поэтому мы ограничились дополнениями к уже имеющимся обзорам и собственными комментариями, среди которых и наше решение задачи И. М. Гельфанда об установлении прямой связи формул типа Крейна и типа Гельфанда-Левитана.

В дополнении 2 мы коротко остановились на родственно близком теории следов разделе спектральной теории - теории $\zeta$-функции, некоммутативном вычете и спектральной асимметрии. В некоторых случаях мы сочли необходимым сделать поправки к устоявшейся истории вопроса.

Известная часть современной теории индекса линейных операторов - это применение теории регуляризованных следов. Так как это тоже хорошо освещенный в литературе вопрос, мы сочли возможным ограничиться коротким дополнением 3. Укажем еще на очень тесную связь этого материала с содержанием предыдущего дополнения 2.

Дополнение 4 посвящено обсуждению результатов одного из малоизученных (хотя исторически одного из самых первых) приложений теории регуляризованных следов - приближенному вычислению с помощью следов первых собственных чисел оператора.

В дополнении 5 мы осветили некоторые нестандартные, но весьма любопытные работы. Некоторые из обсуждаемых работ могут, на наш взгляд, послужить источником интересных задач.

Наконец, в дополнении 6 мы предлагаем наиболее интересные и актуальные нерешенные задачи. Конечно, этот перечень несет на себе отпечаток наших личных взглядов и вкусов, но мы надеемся, что это дополнение поможет более быстрому развитию теории.

Мы не затрагиваем формулу следа Сельберга, так как, несмотря на название, эта теория не имеет отношения к теме обзора. Формула следа Сельберга это формула суммирования, далеко обобщающая формулу суммирования Пуассона, и хотя обычно ее выводят через вычисление следа подходящего ядерного оператора, в ней нет пары операторов и она не может быть названа регуляризованным следом. Эта теория может быть использована как инструмент в теории регуляризованных следов, подобно использованию формулы Пуассона для исследования спектра генераторов периодических потоков на многообразиях, и имея в виду эту возможность, дадим ссылку на достаточно содержательный обзор [25].

При работе над обзором мы взяли за основной принцип обязательность подробного освещения оригинальных методов, технических тонкостей, а часто и постановок задач на примере хотя бы одной работы по соответствующей теме. Далеко не всегда при этом наш выбор падал на работу с последним, наиболее сильным результатом. Часто более ранние работы с простыми, незагроможденными формулировками прозрачнее и лучше подходят для таких целей. Также мы старались привести в работе как можно больше явно вычисленных формул следов. Зачастую один их внешний вид, сравнение с аналогами, наблюдение за устойчиво повторяющимися элементами и на их фоне за новыми составляющими говорят больше, чем строгие доказательства. Кроме того, мы считаем 
необходимым собрать эти формулы, разбросанные по первоисточникам, в одном обзоре.

Мы ограничили время выхода работ, охваченных обзором, 2005 годом, но, к сожалению, уверенности в полном охвате всех работ за последние несколько лет у нас нет. Также мы не можем исключить и того, что в поле нашего зрения (особенно с учетом объема материала) не попали и отдельные содержательные более ранние работы. Во всех таких случаях мы приносим свои извинения и авторам, и читателям, и будем признательны всем за указания на такие работы. Мы внесем соответствующие добавления и поправки при первом удобном случае.

\section{Регуляризованные следы. Обыкновенные дифференциальные операторы}

Этот раздел мы начнем с изложения пионерской работы И. М. Гельфанда и Б. М. Левитана [7]. Формула регуляризованного следа (4) была получена авторами аналитическим методом из асимптотического поведения решения задачи Коши. Воспользовавшись равенством (абсолютная сходимость ряда известна из асимптотики $\mu_{n}$ )

$$
\begin{aligned}
\sum_{n=1}^{\infty}\left(\lambda_{n}-\mu_{n}\right) & =\lim _{\zeta \rightarrow+\infty} \zeta^{2} \sum_{n=1}^{\infty}\left(\left(\zeta+\mu_{n}\right)^{-1}-\left(\zeta+\lambda_{n}\right)^{-1}\right) \\
& =\lim _{\zeta \rightarrow+\infty} \zeta^{2} \int_{0}^{\pi}\left(G_{0}(x, x, \zeta)-G(x, x, \zeta)\right) d x,
\end{aligned}
$$

где $G(x, t, \zeta)$ - функция Грина задачи $(3)$, а $G_{0}(x, t, \zeta)$ - функция Грина той же задачи с $q(x) \equiv 0$, авторы получили

$$
\sum_{n=1}^{\infty}\left(\lambda_{n}-\mu_{n}\right)=\lim _{\zeta \rightarrow \infty} \zeta^{2}\left(\frac{d}{d \zeta} \ln u_{0 x}^{\prime}(\pi, \zeta)-\frac{d}{d \zeta} \ln u_{x}^{\prime}(\pi, \zeta)\right),
$$

где $u(x, \zeta)$ - решение уравнения $-u^{\prime \prime}+(q(x)+\zeta) u=0$ с начальными условиями $u(0)=1, u^{\prime}(0)=0$, а $u_{0}(x, \zeta)$ - аналогичное решение для $q(x) \equiv 0$ (т.е. $\left.u_{0}(x, \zeta)=\operatorname{ch} \sqrt{\zeta} x\right)$. Далее с помощью представления оператором преобразования решения возмущенной задачи через решение невозмущенной они исследовали асимптотическое поведение $\left(\ln u_{x}^{\prime}(\pi, \zeta)\right)_{\zeta}^{\prime}$ и получили ответ.

След резольвенты оператора был вскоре использован для исследования регуляризованных следов одним из авторов этой работы вновь [26], и этот метод до сих пор остается одним из основных в теории, а вот то, что ответ содержится в асимптотике характеристического определителя, оставалось незамеченным в течении длительного времени.

Формула (4) не имела вида (6), содержащего оператор возмущения, ни в окончательном виде, ни на стадии промежуточных результатов, и долгое время это положение дел было типичным для получаемых многими авторами результатов по следам для ДО, но именно для первой формулы Гельфанда-Левитана почти сразу Л. А. Дикий дал другое доказательство, показав в работе [8], что формула (4) фактически есть формула (6). Пусть $L_{0}-$ оператор вида (3) с условиями Дирихле и с $q(x) \equiv 0$. Собственные числа $L_{0}$ суть $\lambda_{n}=n^{2}, n=1,2, \ldots$, 
а нормированные собственные функции $\psi_{n}=\sqrt{2 / \pi} \sin n x, n=1,2, \ldots$. Также рассмотрим оператор $L=L_{0}+q$, где $q$ есть оператор умножения на неотрицательную вещественнозначную функцию $q(x)$. Мы будем, в отличие от оригинальной работы, предполагать, что $q(x) \in L_{2}[0, \pi]$, по мере необходимости уточняя условия на $q(x)$. Собственные числа оператора $L$ обозначим через $\mu_{n}$, нормированные собственные функции - через $v_{n}$. В работе было проведено прямое изучение поведения при $N \rightarrow \infty$ суммы $\sum_{n=1}^{N}\left(\left(v_{n}, L v_{n}\right)-\left(\psi_{n}, L \psi_{n}\right)\right)$ через разложение вектора $\psi_{n}$ по базису $\left\{v_{i}\right\}_{i=1}^{\infty}: \psi_{k}=\sum_{i=1}^{\infty} a_{i k} v_{i}, a_{i k}=\left(v_{i}, \psi_{k}\right)$, и исследование свойств унитарной матрицы $\left\|a_{i k}\right\|$ с использованием известных свойств собственных чисел и собственных функций возмущенного оператора: $\mu_{n}=n^{2}+O(1), v_{n}=\psi_{n}+O(1 / n)$, и доказано, что эта сумма стремится к нулю при $N \rightarrow \infty$. Зафиксируем результат в явном виде:

$$
\sum_{n=1}^{\infty}\left(\mu_{n}-n^{2}-\frac{2}{\pi} \int_{0}^{\pi} q(x) \sin ^{2} n x d x\right)=0 .
$$

Если теперь продолжить исследование формулы (8) при более жестких предположениях о $q(x)$ и просуммировать ряд Фурье, возникающий из использования элементарной формулы $\sin ^{2} n x=(1-\cos 2 n x) / 2$, то получаем:

$$
\sum_{n=1}^{\infty}\left(\mu_{n}-n^{2}-\frac{1}{\pi} \int_{0}^{\pi} q(x) d x\right)=\frac{1}{2 \pi} \int_{0}^{\pi} q(x) d x-\frac{1}{4}(q(0)+q(\pi)) .
$$

Итак, если потенциал $q(x) \in L_{2}[0, \pi]$ таков, что его ряд Фурье сходится в точках 0 и $\pi$ к числам $q(0)$ и $q(\pi)$ соответственно, то верна ${ }^{7}$ формула (9).

Крупный шаг в развитии теории был сделан в 1955 году Л. А. Диким и в 1956 году И. М. Гельфандом, впервые рассмотревшим задачу о вычислении регуляризованных сумм степеней собственных чисел. Методы этих работ были различными, оба метода заняли важнейшее место в арсенале теории.

И. М. Гельфанд [26] рассмотрел асимптотическое поведение следа резольвенты оператора $L$ двумя способами. С одной стороны, $R(-\lambda)$ можно выразить через $R_{0}(-\lambda)$ и оператор умножения на $q$ сходящимся по норме рядом:

$$
(L+\lambda E)^{-1}=R_{0}(-\lambda) \sum_{k=0}^{\infty}(-1)^{k}\left(q \cdot R_{0}(-\lambda)\right)^{k},
$$

и, используя точное выражение для функции Грина оператора $L_{0}$, aвтор дал алгоритм вычисления коэффициентов асимптотического при $\lambda \rightarrow+\infty$ разложения

$$
\operatorname{Tr}(R(-\lambda)) \sim \sum_{n=0}^{\infty} a_{n} \lambda^{-n-1 / 2}
$$

\footnotetext{
${ }^{7} \mathrm{~B}$ оригинальной работе Л.А. Дикого при полностью строго доказанной формуле с вышеприведенными условиями на $q(x)$ сформулировано утверждение для $q(x) \in C^{1}[0, \pi]$. Впоследствии многие авторы в качестве примеров к своим результатам предлагали "усиление" результата Л. А. Дикого. Данная теорема в окончательном виде опубликована в монографии В. А. Марченко по теории операторов Штурма-Лиувилля [27; задача на с. 94]. Содержательные уточнения и обобщения этой теоремы были получены в работах [28]-[32], ниже мы к ним вернемся.
} 
С другой стороны, так как

$$
\operatorname{Tr}(R(-\lambda))=\sum_{n=1}^{\infty}\left(\mu_{n}+\lambda\right)^{-1}
$$

то (при наличии у $\mu_{n}$ достаточно точного асимптотического разложения по степеням $n$, наша запись подразумевает бесконечное разложение) возможно исследование той же асимптотики из второго представления, и Гельфанд получил разложение

$$
\sum_{n=1}^{\infty} \frac{1}{\mu_{n}+\lambda}=\sum_{k=0}^{\infty} \frac{1}{\lambda^{k+1}}\left(\sum_{n=1}^{\infty}\left(\mu_{n}^{k}-A_{k}(n)\right)-\frac{1}{2} c_{0, k}\right)-\frac{\pi \psi^{\prime}(\lambda)}{2 \sqrt{\psi(\lambda)}}+O\left(\lambda^{-\infty}\right) .
$$

Здесь $\psi(\lambda)$ - функция, обратная к монотонной функции $\lambda=\varphi\left(x^{2}\right) \in C^{\infty}$, где $\varphi(n)=\mu_{n}$. Неоднозначность выбора $\varphi$ не выходит за рамки погрешности полученного разложения.

Сравнение полученных разложений и дает искомые формулы следов

$$
\sum_{n=1}^{\infty}\left(\mu_{n}^{k}-A_{k}(n)\right)=B(k)
$$

где $A_{k}(n)$ - отрезок асимптотического разложения $\mu_{n}^{k}$, а $B(k)$ - явно вычисляемое через данные оператора число.

Другой подход предложил в работе [33] Л. А. Дикий. Для резольвенты оператора Штурма-Лиувилля $L$ автор на основе некоторых коммутационных соотношений получил асимптотическое равенство

$$
(L-z)^{-1} \sim \sum_{l=0}^{\infty} \sum_{m=0}^{l} T_{l, m}(-1, x)\left(D^{2}-z\right)^{-1-\frac{l+m}{2}} D^{m}
$$

понимаемое в том смысле, что разность между левой частью и частичной суммой ряда справа до некоторого $N$ есть оператор $R_{N}$ такой, что операторы $R_{N} \cdot D^{2+N}$ и $D^{2+N} \cdot R_{N}$ продолжаются до ограниченного. Для коэффициентов этой асимптотики были найдены рекуррентные формулы

$$
\begin{gathered}
T_{l, m}(k, x)=R_{l, m}(x) \cdot\left(\begin{array}{c}
k \\
\frac{l+m}{2}
\end{array}\right), \\
R_{l+2, m}(x)=q(x) R_{l, m}(x)-R_{l, m}^{\prime \prime}(x)+2 i R_{l+1, m-1}^{\prime}(x),
\end{gathered}
$$

где $R_{0,0}(x) \equiv 1, R_{l, m}(x) \equiv 0$ при $m>l, R_{l, m}(x) \equiv 0$, если $l+m$ - нечетное. Используя полученное представление резольвенты, Дикий определил комплексные степени $s, \operatorname{Re} s<-1$, оператора $L$ :

$$
L^{s} \sim \sum_{l=0}^{\infty} \sum_{m=0}^{l} T_{l, m}(-1, x)\left(\begin{array}{c}
s \\
\frac{l+m}{2}
\end{array}\right) D^{2 s-l}
$$

где ряд асимптотический в прежнем смысле. Для произвольного $s$ степень определяется равенством $L^{s}=L^{s-k} L^{k}$ при любом подходящем $k$. Теперь стало 
возможно использовать $\zeta$-функцию оператора для нахождения формул следов: при $\operatorname{Re} s>1$

$$
\begin{aligned}
Z(s) & =\sum_{n=1}^{\infty} \mu_{n}^{-s}=\sum_{n=1}^{\infty}\left(L^{-s} \sqrt{\frac{2}{\pi}} \sin n x, \sqrt{\frac{2}{\pi}} \sin n x\right) \\
& =\frac{2}{\pi} \sum_{l=0}^{\infty} \sum_{n=1}^{\infty} \int_{0}^{\pi} \sum_{m=0}^{l} T_{l, m}(-1, x)\left(\begin{array}{c}
-s \\
\frac{l+m}{2}
\end{array}\right) \frac{1}{n^{2 s+2 l}}(\sin n x)^{(l)} \sin n x d x .
\end{aligned}
$$

Исследовав поведение при $n \rightarrow \infty$ интегралов вида $\int_{0}^{\pi} f(x) \sin n x d x$ и перенеся несколько старших членов влево, автор аналитически продолжил $Z(s)$ в полуплоскость, содержащую точки $s=-1,-2, \ldots$ и получил формулы $(10)$.

В работе [34] Л.А. Дикий сделал некоторую систематизацию и сравнение имевшихся к тому моменту методов в теории следов, а также впервые указал на глубокую связь теории следов с теорией возмущений дискретного спектра. Из теории возмущений для изолированного простого собственного числа известно, что верно представление

$$
\mu_{n}(\varepsilon)=\lambda_{n}+\varepsilon \lambda_{n}^{(1)}+\varepsilon^{2} \lambda_{n}^{(2)}+\cdots,
$$

где $\lambda_{n}$ - собственные числа невозмущенного оператора $A, \mu_{n}(\varepsilon)$ - собственные числа оператора $A+\varepsilon B$, а $\lambda_{n}^{(i)}$ - поправки теории возмущений, например

$$
\lambda_{n}^{(1)}=\left(B \varphi_{n}, \varphi_{n}\right), \quad \lambda_{n}^{(2)}=\sum_{m: \lambda_{m} \neq \lambda_{n}} \frac{\left(B \varphi_{n}, \varphi_{m}\right)\left(B \varphi_{m}, \varphi_{n}\right)}{\lambda_{n}-\lambda_{m}}
$$

здесь $\varphi_{n}$ - собственные векторы невозмущенного оператора. Для регулярного оператора Штурма-Лиувилля при некоторых предположениях о потенциале Л. А. Дикий доказал, что

$$
\sum\left(\mu_{n}-\lambda_{n}-\lambda_{n}^{(1)}\right)=0 \quad \text { и } \quad \sum\left(\mu_{n}-\lambda_{n}-\lambda_{n}^{(1)}-\lambda_{n}^{(2)}\right)=0 .
$$

Аналогичный результат был им доказан и для квадрата этого оператора.

Несколько позже Б. М. Левитан [35] предложил еще один метод для вычисления следов оператора Штурма-Лиувилля: приравнивая выражения для характеристического определителя через решение подходящей задачи Коши, с одной стороны, и через соответствующее бесконечное произведение с другой, автор находит и сравнивает коэффициенты асимптотических разложений этих выражений, из чего и получает формулы следов.

Исследование сингулярного оператора Штурма-Лиувилля, проведенное в 1957 году Л.Д. Фаддеевым [12], вывело теорию следов на содержательно новый класс задач и впервые установило некоторую связь формул типа Гельфанда-Левитана и Крейна. Этот аспект мы обсудим в дополнении 2, а саму работу [12] и большой массив работ, ею инициированный, мы обсудим в основном тексте, так как и по идейному содержанию, и по технике это направление относится к формулам типа Гельфанда-Левитана.

В [12] Л. Д. Фаддеев рассмотрел в $L_{2}[0,+\infty)$ пару операторов $H_{i}, i=1,2$ :

$$
-y^{\prime \prime}+q_{i}(x) y=\lambda y, \quad y(0)=0,
$$


где выполнены следующие условия на потенциалы:

$$
\int_{0}^{\infty} x\left|q_{i}(x)\right| d x<\infty, \quad i=1,2 ; \quad \int_{0}^{\infty}\left(q_{1}(x)-q_{2}(x)\right) d x=0,
$$

и $q_{1}(x)-q_{2}(x)$ обладает определенной гладкостью в некоторой окрестности нуля. При этих условиях Л. Д. Фаддеев доказал, что существует предел

$$
\lim _{\Lambda \rightarrow \infty} \int_{-\infty}^{\Lambda} \lambda d \operatorname{Tr}\left(E_{\lambda}^{(1)}-E_{\lambda}^{(2)}\right)
$$

здесь $E_{\lambda}^{(i)}$ - спектральная функция оператора $H_{i}$, и, вычислив этот предел двумя способами, получил тождество 8

$$
\sum_{l=1}^{n_{1}} \lambda_{l}^{(1)}-\sum_{l=1}^{n_{2}} \lambda_{l}^{(2)}+\frac{2}{\pi} \int_{0}^{\infty} k\left(\eta_{1}(k)-\eta_{2}(k)\right) d k=-\frac{1}{4}\left(q_{1}(0)-q_{2}(0)\right),
$$

где $\lambda_{l}^{(i)}$ - отрицательные собственные числа операторов, $\eta_{i}(k)$ - соответствующие предельные фазы. Отметим, что предел (12) в применении к оператору (3) (с естественным ограничением $\Lambda \notin \bigcup\left[\lambda_{n}, \mu_{n}\right]$ ) дает в точности формулу следа Гельфанда-Левитана, и полученная Л. Д. Фаддеевым формула является формулой первого регуляризованного следа в том же смысле. В этой же работе получен результат для матричного случая. Подчеркнем, что (13) - первая явная формула следа для оператора с непрерывным спектром.

Вскоре В. С. Буслаев и Л. Д. Фаддеев [13] в той же задаче получили формулы следов всех порядков, например, так выглядит второй след (при $\left.q_{2}(x) \equiv 0\right)$ :

$$
\begin{aligned}
\sum_{l=1}^{m} \lambda_{l}^{2} & +\int_{0}^{\infty}\left(\frac{4 \eta(t) t^{3}}{\pi}-\frac{2 t^{2}}{\pi} \int_{0}^{\infty} q(\tau) d \tau-\frac{q^{\prime}(0)}{2 \pi}-\frac{1}{2 \pi} \int_{0}^{\infty} q^{2}(\tau) d \tau\right) d t \\
& =\frac{q^{\prime \prime}(0)-2 q^{2}(0)}{8} .
\end{aligned}
$$

Здесь же было найдено явное выражение функции спектрального сдвига $\xi(\lambda)$ (см. дополнение 1) через предельную фазу:

$$
\xi(\lambda)= \begin{cases}\frac{1}{\pi} \eta(\sqrt{\lambda}), & \lambda>0, \\ -\int_{-\infty}^{\lambda} \sum_{l} \delta\left(z-\lambda_{l}\right) d z, & \lambda<0 .\end{cases}
$$

Метод работы можно отнести к методу $\zeta$-функций, и в каком-то смысле здесь дана первая конструкция $\zeta$-функции оператора с непрерывным спектром.

Первый результат для операторов высокого порядка был получен в работе Ч. Хальберга и В. Крамера [36], здесь впервые после [8] был использован подход на основе исследования абстрактных операторов и для возмущения ограниченным оператором $V$ дискретного самосопряженного оператора $T$ такого, что $T^{-1}$

\footnotetext{
${ }^{8}$ Напомним: решение задачи Коши $\varphi(0)=0, \varphi^{\prime}(0)=1$ уравнения (11) имеет асимптотику $\varphi(x, \sqrt{\lambda})=A(\sqrt{\lambda}) \sin (\sqrt{\lambda} x-\eta(\sqrt{\lambda}))+o(1)$ при $x \rightarrow+\infty$, и функция $\eta(\sqrt{\lambda})$ называется предельной фазой.
} 
ядерный, в предположении, что сходятся ряды $\sum\left(\lambda_{n}-\mu_{n}\right)$ и $\sum\left(V \varphi_{n}, \varphi_{n}\right)$, доказано равенство $\sum\left(\lambda_{n}-\mu_{n}\right)=\sum\left(V \varphi_{n}, \varphi_{n}\right)$, где $\left\{\varphi_{n}\right\}$ - собственные векторы $T$. $\mathrm{B}$ качестве первого примера в работе впервые был найден след для оператора второго порядка на конечном отрезке с нераспадающимися краевыми условиями, вторым примером стал след для оператора 4-го порядка, однако формула вычислена неверно. В работах Р. Гилберта и В. Крамера (см. [9]) для самосопряженных $T$ и $V$ таких, что $T^{-1}, V T^{-1} \in \mathfrak{S}_{1}$, были получены формулы регуляризованных следов с регуляризацией, состоящей из нескольких поправок теории возмущений, техника исследования следа разности резольвент была доведена при некоторых условиях на операторы до формулы

$$
\lim _{\operatorname{Im} \lambda \rightarrow \infty}(\operatorname{Im} \lambda)^{2}\left(\operatorname{Tr}\left(R-R_{0}\right)+\operatorname{Tr}\left(R_{0} V R\right)\right)=0,
$$

которая была применена к исследованию задачи (11), и были получены те же результаты, что и в [12], и им родственные, с некоторыми уточнениями условий на потенциал.

Первой работой, в которой был рассмотрен сингулярный ДО с дискретным спектром, стала работа М. Г. Гасымова [10]. Для произвольного оператора $A$ автор ввел понятие следа в базисе: если для некоторого ортонормированного базиса пространства $\left\{\varphi_{n}\right\}$ существует предел $\lim _{N \rightarrow \infty} \sum_{n=1}^{N}\left(A \varphi_{n}, \varphi_{n}\right)$, то он называется следом $A$ в базисе $\left\{\varphi_{n}\right\}$. Основным результатом работы стала теорема: пусть $A$ - самосопряженный дискретный оператор, $B$ - самосопряженный оператор такой, что $A+B$ - самосопряженный дискретный, и пусть существуют и совпадают следы $B$ в двух базисах из собственных векторов $A$ и $A+B$. Тогда $\sum_{n=1}^{\infty}\left(\mu_{n}-\lambda_{n}\right)=\sum_{n=1}^{\infty}\left(B \varphi_{n}, \varphi_{n}\right)$, где $\mu_{n}$ и $\lambda_{n}-$ собственные числа $A+B$ и $A$. $\mathrm{B}$ качестве приложений в [10] были рассмотрены две задачи для обыкновенных полуограниченных ДО второго порядка на оси и полуоси с дискретным спектром, возмущенных оператором умножения на финитную функцию $q(x)$ с нулевым средним, и на оси доказана сходимость ряда разностей собственных чисел к нулю, а для задачи на полуоси с дополнительным требованием дифференцируемости $q(x)$ в некоторой окрестности нуля доказана формула

$$
\sum_{n=1}^{+\infty}\left(\mu_{n}-\lambda_{n}\right)=\frac{q(0)}{4}
$$

Сразу вслед за этой работой вышла работа М.Г. Гасымова и Б. М. Левитана [37], в которой в задаче на полуоси был рассмотрен случай различных краевых условий у пары операторов и получена формула

$$
\lim _{\lambda \rightarrow \infty}\left(\sum_{\mu_{n}<\lambda}\left(\mu_{n}-\lambda_{n}\right)-\frac{2}{\pi}\left(h_{1}-h_{2}\right) \sqrt{\lambda}\right)=-\frac{1}{2}\left(h_{2}^{2}-h_{1}^{2}\right)+\frac{p(0)}{4},
$$

здесь $h_{1}, h_{2}$ - параметры краевых условий. На тот момент это был первый результат такого типа. Аналогичные "формулы следа" в трехмерном случае получил С. И. Гринберг [38], изучивший поведение сумм разностей собственных чисел $\lambda_{k}$ и $\mu_{k}$ первой и третьей краевых задач для оператора Лапласа в области, приведем две полученные им формулы:

$$
8 \pi \sum_{\lambda_{k} \leqslant x} \frac{\lambda_{k}-\mu_{k}}{\lambda_{k}^{2}} \sim s \ln x, \quad \sum_{k=1}^{n} \frac{\mu_{k}}{\lambda_{k}}=n-s \sqrt[3]{\frac{9 \pi n^{2}}{128 v^{2}}}+o\left(n^{\frac{2}{3}}\right),
$$


здесь $v$ - объем области, а $s$ - площадь ограничивающей ее поверхности.

Работы Р. Ф. Шевченко (см. [39]) были одними из первых посвященных формулам следа для двучленной операции высокого порядка $d^{n} / d x^{n}+q(x)$ на отрезке.

Крупное продвижение в этой теории принадлежит А.Г. Костюченко [40]. Для возмущения положительного дискретного ДО в $L_{2}(\mathbb{R})$ с операцией вида

$$
l y=(-1)^{m} y^{(2 m)}+p_{2 m-2}(x) y^{(2 m-2)}+\cdots+p_{0}(x) y
$$

оператором умножения на финитную функцию $q(x) \in L_{1}$ было доказано, что если потенциал имеет нулевое среднее, то $\sum_{n=1}^{+\infty}\left(\mu_{n}-\lambda_{n}\right)=0$, и был получен результат для оператора четвертого порядка на полуоси: для граничной задачи $y(0)=y^{\prime}(0)=0$ и для потенциала $q(x)$, имеющего, сверх уже указанных условий, ограниченную вариацию в некоторой окрестности нуля, верна формула (16).

В работе В. А. Садовничего [41] исследование операторов высокого порядка было проведено новым методом - применением $\theta$-функции оператора. Суть метода в следующем. Так как (для полуограниченных операторов)

$$
\operatorname{Tr}\left(e^{-t L_{0}}\right)=\sum_{n=1}^{\infty} e^{-\lambda_{n} t}, \quad \operatorname{Tr}\left(e^{-t L}\right)=\sum_{n=1}^{\infty} e^{-\mu_{n} t},
$$

где $\left\{\lambda_{n}\right\}$ и $\left\{\mu_{n}\right\}$ - собственные числа операторов $L_{0}$ и $L$, то формально

$$
\lim _{t \rightarrow 0+}\left(\operatorname{Tr}\left(e^{-t L_{0}}\right)-\operatorname{Tr}\left(e^{-t L}\right)\right)_{t}^{\prime}=\lim _{t \rightarrow 0+} \sum_{n=1}^{\infty}\left(\mu_{n} e^{-\mu_{n} t}-\lambda_{n} e^{-\lambda_{n} t}\right)=\sum_{n=1}^{\infty}\left(\mu_{n}-\lambda_{n}\right),
$$

и если мы при каких-либо условиях найдем предел слева, доказав сходимость ряда справа, то получим формулу следа. В работе [41] для оператора $L_{0}$, определяемого

дифференциальным выражением $(-1)^{m} y^{(2 m)}$ и

краевыми условиями $y(0)=\cdots=y^{(m-1)}(0)=y(\pi)=\cdots=y^{(m-1)}(\pi)=0$,

автор исследовал $\operatorname{Tr}\left(e^{-t L_{0}}\right)$ при $t \rightarrow 0+$, далее изучил асимптотическое поведение функции Грина $G(x, y, t)$ оператора $L$, определяемого дифференциальным выражением $(-1)^{m} y^{(2 m)}+p(x) y$ и теми же краевыми условиями, и доказал, что при $p(x) \in C^{1}$ и $\int_{0}^{\pi} p(x) d x=0$ верно

$$
\lim _{t \rightarrow 0+} \int_{0}^{\pi}\left(G_{0 t}^{\prime}(x, x, t)-G_{t}^{\prime}(x, x, t)\right) d x=\lim _{t \rightarrow 0+} \int_{0}^{\pi} p(x) G_{0}(x, x, t) d x .
$$

Сходимость ряда разностей собственных чисел при сделанных предположениях следует из асимптотических формул для $\mu_{n}$, и мы получаем формулу следа. Например, для оператора четвертого порядка имеем $\sum_{n=1}^{\infty}\left(\mu_{n}-\lambda_{n}\right)=-(p(0)+$ $p(\pi)) / 4$.

Далее, в работе [42] В. А. Садовничий, развив метод работы [33] построения $\zeta$-функции оператора через построение его комплексных степеней (теории ПДО еще не существовало), нашел формулы следов для обыкновенных операторов 
вида $\sum_{j} p_{2 m-2 j}(x) y^{(2 m-2 j)}$. Наконец, он же в работе [43] нашел след для системы Дирака, вновь построив комплексные степени оператора. Мы приведем формулу первого следа для системы Дирака, полученную чуть позже в более общей постановке Э. Абдукадыровым [44] методом, восходящим к работе [35]. Для собственных чисел задачи

$$
\begin{gathered}
\left\{\begin{array}{l}
u_{2}^{\prime}+p(x) u_{1}=\lambda u_{1}, \\
-u_{1}^{\prime}+q(x) u_{2}=\lambda u_{2},
\end{array}\right. \\
u_{2}^{\prime}(0) \cos \alpha+u_{2}(0) \sin \alpha=u_{2}^{\prime}(\pi) \cos \beta+u_{2}(\pi) \sin \beta=0
\end{gathered}
$$

имеет место формула ${ }^{9}$ :

$$
\lambda_{0}+\sum_{n=1}^{\infty}\left(\lambda_{n}+\lambda_{-n}\right)=\frac{q(\pi)-p(\pi)}{4} \cos 2 \beta+\frac{q(0)-p(0)}{4} \cos 2 \alpha
$$

верная при выполнении некоторых обычных условий на коэффициенты $p(x)$ и $q(x)$ типа гладкости и обнуления средних.

Фундаментальные результаты теории регуляризованных следов были получены в работе В.Б. Лидского и В.А. Садовничего [11]. Авторы создали метод получения регуляризованных сумм корней целых функций одного класса и применили его к получению следов обыкновенных ДО. Рассмотрим целую функцию $f(z)$, представимую в виде

$$
f(z)=\sum_{k=0}^{n} e^{\alpha_{k} z} P_{k, N}(z)
$$

где $\alpha_{k} \in \mathbb{C}, P_{k, N}(z)=z^{n_{k}} \sum_{l=0}^{N} \beta_{l}^{(k)} z^{-l}+o\left(z^{n_{k}-N}\right),|z| \rightarrow \infty$, причем $\mathbb{C}$ можно покрыть конечным числом открытых секторов, в каждом из которых функции $P_{k, N}(z)$ являются аналитическими вне круга достаточно большого радиуса. Такие $f(z)$ называются функциями класса $K$. Будем считать, что разложение функций $P_{k, N}(z)$ имеет место для любого $N$, и индекс $N$ будем опускать. Обозначим выпуклую оболочку точек $\bar{\alpha}_{0}, \ldots, \bar{\alpha}_{n}(18)$ через $R$, это $r$-угольник, $r \leqslant n$, пусть в вершинах лежат $\bar{\alpha}_{0}, \ldots, \bar{\alpha}_{r-1}$. Удалим из плоскости $r$ секторов малого раствора с биссектрисами, параллельными внешним нормалям к $R$, и вершинами в $z=0$, оставшееся множество (обозначим его $\Omega$ ) имеет вид объединения $r$ открытых секторов $\Omega_{s}, s=0, \ldots, r-1$. Выберем теперь в одном из секторов $\Omega_{s}$ (пусть $\Omega_{0}$ ) луч $l$ и построим контур $\Gamma_{0}$, состоящий из дважды проходимого луча $l$ и окружности $\gamma$ с центром в нуле, так, что все нули $f(z)$ лежат вне $\Gamma_{0}$ (если $f(0)=0$, то без смены обозначений рассмотрим $f(z) / z^{s}$, где $s-$ кратность корня $z=0)$. Функция

$$
Z_{0}(\sigma)=\frac{1}{2 \pi i} \int_{\Gamma_{0}} z^{-\sigma} \frac{f^{\prime}(z)}{f(z)} d z
$$

называется ל-функцией, ассоциированной с функцией $f(z)$. Здесь $z^{-\sigma}=$ $e^{-\sigma \operatorname{Ln} z}$, где $\operatorname{Ln} z$ - фиксированная регулярная во внешности $\Gamma_{0}$ ветвь

\footnotetext{
${ }^{9}$ Напомним, что собственные числа системы Дирака неограничены как сверху, так и снизу.
} 
логарифма. Интеграл сходится при $\operatorname{Re} \sigma>1$, и при этих $\sigma \quad Z_{0}(\sigma)=\sum_{l} z_{l}^{-\sigma}$, здесь $\left\{z_{l}\right\}$ - все нули $f(z)$. Отметим: $Z_{0}(\sigma)$ аналитически продолжается во всю $\sigma$-плоскость как целая функция. Данный результат неожиданен - к тому времени в спектральной теории $\zeta$-функция оператора была известным и широко используемым объектом, и было хорошо известно, что $\zeta$-функция оператора всегда мероморфна. Мы вернемся к этому вопросу в дополнении 2, а пока продолжим анализ результатов работы [11]: один из центральных результатов этой работы - вычисленные значения аналитического продолжения $Z_{0}(\sigma)$ : при $m=0,1,2, \ldots$

$$
Z_{0}(-m)=\omega_{m+1}^{(0)},
$$

где $\omega_{m}^{(0)}-$ коэффициенты разложения

$$
\frac{f^{\prime}(z)}{f(z)}=\alpha_{0}+\frac{P_{0}^{\prime}(z)}{P_{0}(z)}+O\left(e^{\delta|z|}\right) \sim \sum_{\nu=0}^{\infty} \frac{\omega_{\nu}^{(0)}}{z^{\nu}}
$$

при $z \in \Gamma_{0},|z| \rightarrow \infty$.

С другой стороны, корни $f(z)$ распадаются на $r$ серий $z_{n, s}=a_{s} n(1+o(1))$, $a_{s}=2 \pi i /\left(\alpha_{s+1}-\alpha_{s}\right)$. Поведение $z_{n, s}$ уточняется методом последовательных приближений. Если на границе $R$ лежат только $\bar{\alpha}_{0}, \ldots, \bar{\alpha}_{r-1}$, то

$$
z_{n, s} \sim a_{s} n\left(1+b_{s} \frac{\ln n}{n}+\frac{c_{s}}{n}+\sum_{k=1}^{\infty} \frac{R_{k}^{(s)}(\ln n)}{n^{k+1}}\right),
$$

здесь $R_{k}^{(s)}(\cdot)$ - многочлены степени $k$. Если на границе $R$ лежат другие $\bar{\alpha}_{s}$ и делят его сторону на соизмеримые части, то верны аналогичные формулы с разложением по дробным степеням $n$. Теперь возведем (20) в степень $-\sigma$ :

$$
z_{n, s}^{-\sigma} \sim \sum_{k=0}^{\infty} \frac{Q_{k}^{(s)}(\sigma, \ln n)}{n^{k+\sigma}}, \quad \text { где } \quad Q_{k}^{(s)}(\sigma, \ln n)=\sum_{\nu=0}^{k} d_{k \nu}^{(s)}(\sigma) \ln ^{\nu} n,
$$

a $d_{k \nu}^{(s)}(\cdot)$ - полиномы. Фиксируем натуральное $\tau$. Функция

$$
\Psi_{\tau}(\sigma)=\sum_{n} \sum_{s=0}^{r-1}\left(z_{n, s}^{-\sigma}-\sum_{k=0}^{\tau} \frac{Q_{k}^{(s)}(\sigma, \ln n)}{n^{k+\sigma}}\right)
$$

аналитически продолжается в полуплоскость $\operatorname{Re} \sigma>-\tau$. Вычислим $\Psi_{\tau}(-m)$, $m<\tau$, называемые регуляризованными $m$-суммами корней $f(z)$. Введем в рассмотрение функцию

$$
\Phi_{\tau}(\sigma)=\sum_{n=1}^{\infty} \sum_{s=0}^{r-1} \sum_{k=0}^{\tau} \frac{Q_{k}^{(s)}(\sigma, \ln n)}{n^{k+\sigma}},
$$

регулярную при $\operatorname{Re} \sigma>1$. Тогда $\Psi_{\tau}(\sigma)=Z_{0}(\sigma)-\Phi_{\tau}(\sigma)$.

Так как $Z_{0}(\sigma)$ - целая функция, то $\Phi_{\tau}(\sigma)$ продолжается в полуплоскость $\operatorname{Re} \sigma>-\tau$ вместе с $\Psi_{\tau}(\sigma)$. Но $\Phi_{\tau}(\sigma)$ выражается через $\zeta$-функцию Римана:

$$
\Phi_{\tau}(\sigma)=\sum_{k=0}^{\tau} \sum_{\nu=0}^{k}\left(\sum_{s=0}^{r-1} a_{s}^{-\sigma} d_{k \nu}^{(s)}(\sigma)\right) \sum_{n=1}^{\infty} \frac{\ln ^{\nu} n}{n^{k+\sigma}}=\sum_{k=0}^{\tau} \sum_{\nu=0}^{k} D_{k \nu}(\sigma)(-1)^{\nu} \zeta^{(\nu)}(k+\sigma),
$$


и тогда при любом целом $m<\tau$

$$
\sum_{n} \sum_{s=0}^{r-1}\left(z_{n, s}^{m}-\sum_{k=0}^{\tau} \frac{Q_{k}^{(s)}(-m, \ln n)}{n^{k-m}}\right)=\omega_{m+1}^{(0)}-\widetilde{\Phi}_{\tau}(-m),
$$

где $\widetilde{\Phi}_{\tau}(-m)$ определяются истинным началом суммирования по $n$. Дело в том, что асимптотические соотношения (20) задают нумерацию $z_{n, s}$, не обязательно совпадающую с нумерацией по возрастанию модулей. Если есть лишние числа $z_{n, s}$, то $\widetilde{\Phi}_{\tau}(-m)=\Phi_{\tau}(-m)$, если же лишними являются несколько выражений $\sum_{k=0}^{\tau} Q_{k}^{(s)}(\sigma, \ln n) / n^{k+\sigma}$, то соответствующее число надо вычесть и получить $\widetilde{\Phi}_{\tau}(-m)$. Эта разница в нумерации называется дефектом регуляризации, аналогичный объект А. Зоммерфельд [45] назвал квантовым дефектом. Он теснейшим образом связан с таким важнейшим понятием теории операторов, как индекс нётерова оператора, мы вернемся к этому вопросу в дополнении 4, а пока покажем, как авторы [11] вычислили дефект регуляризации. Так как $Q_{k}^{(s)}(0, \ln n)=1, k \geqslant 1$, и $Q_{0}^{(s)}(0, \ln n)=1$, то если в формуле следа положить $m=0$, мы получим целое число, равное дефекту регуляризации:

$$
\varkappa=\omega_{1}^{(0)}+\frac{r}{2}-\sum_{s=0}^{r-1} b_{s} \operatorname{Ln} a_{s}+\sum_{s=0}^{r-1} c_{s} .
$$

Фундаментальная важность этих исследований для теории операторов следует из того, что характеристический определитель обыкновенного ДО с регулярными краевыми условиями (см. [46]) является функцией класса $K$. Более того, можно заметить, что это утверждение верно и для характеристического определителя более общих задач, у которых коэффициенты уравнения и краевых условий зависят от спектрального параметра полиномиально. Ниже мы увидим многочисленные применения этого метода и далеко идущие, подчас совсем не очевидные, обобщения.

Авторы метода сразу же продемонстрировали силу предложенного ими подхода, рассмотрев [47] важную задачу математической физики, нахождение регуляризованных следов для которой ранее было немыслимо. Задача

$$
\begin{gathered}
\left(\frac{d^{2}}{d x^{2}}-\alpha^{2}\right)^{2} u=i \alpha R\left((p(x)-c)\left(\frac{d^{2}}{d x^{2}}-\alpha^{2}\right)^{2} u-p^{\prime \prime}(x) u\right), \\
u(0)=u^{\prime}(0)=u(1)=u^{\prime}(1)=0,
\end{gathered}
$$

известная как один из вариантов задачи Орра-Зоммерфельда, возникает в теории гидродинамической устойчивости, в которой все внимание сосредоточено на малых по модулю собственных числах $c_{l}$, рассматриваемых как функции $\alpha$ и $R$. От их расположения на комплексной плоскости зависит устойчивость соответствующего течения жидкости. В этой работе были детально исследованы линейно независимые решения этого уравнения, доказано, что характеристический определитель задачи является функцией класса $K$. Здесь впервые был исследован случай, когда один из корней этого определителя кратный. Авторы получили формулы асимптотического поведения собственных чисел задачи и доказали формулы регуляризованных следов всех порядков. Подчеркнем, 
что в работе для всех встречающихся коэффициентов были найдены точные рекуррентные соотношения, и формулы следов можно точно выписать, следуя четкому алгоритму. Приведем формулу первого следа. Занумеровав собственные числа $\left\{c_{l}\right\}$ по возрастанию модулей одним индексом и обозначив через $\lambda_{l}$ собственные числа задачи в случае $p(x) \equiv 0$, мы получим

$$
\sum_{l=1}^{\infty}\left(c_{l}-\lambda_{l}\right)=-\frac{3}{4}(p(1)+p(0))+\frac{1}{\alpha \operatorname{sh} \alpha} \int_{0}^{1} \operatorname{sh} \alpha(1-t) \cdot \operatorname{sh} \alpha t \cdot p^{\prime \prime}(t) d t,
$$

из чего извлекаем любопытное следствие: при том, что исходная задача существенно несамосопряженная, имеем $\sum_{l=1}^{\infty}\left(\operatorname{Im} c_{l}-\operatorname{Im} \lambda_{l}\right)=0$.

В работах В.А. Садовничего (см. [48]) был изучен вопрос о нахождении регуляризованных сумм корней функций класса $K$ из одного сектора $T_{s}$. Этот вопрос тесно связан с уже упоминавшимся "противоречием" между мероморфностью $\zeta$-функции оператора и голоморфностью на плоскости $\zeta$-функции, ассоциированной с функцией класса $K$. Рассмотрим пару $\zeta$-функций $(19) Z_{s}(\sigma)$, $Z_{s+1}(\sigma)$, ассоциированных с данной функцией $f(z)$, и введем в рассмотрение следующую функцию:

$$
\zeta_{s}(\sigma)=\frac{Z_{s+1}(\sigma)-Z_{s}(\sigma)}{e^{2 \pi i \sigma}-1}=\frac{1}{2 \pi i} \int_{C_{s}} z^{-\sigma} \frac{f^{\prime}(z)}{f(z)} d z, \quad \operatorname{Re} \sigma>1,
$$

где $C_{s}$ - контур, состоящий из верхних берегов разрезов $\Gamma_{s}$ и $\Gamma_{s+1}$, обращенных к сектору $T_{s}$. Видим, что $\zeta_{s}(\sigma)$ - меромофная функция с простыми полюсами в точках $\sigma=1,0,-1,-2, \ldots$ и

$$
\operatorname{res}_{\sigma=-k} \zeta_{s}(\sigma)=\frac{\omega_{k+1}^{(s+1)}-\omega_{k+1}^{(s)}}{2 \pi i},
$$

а также нетрудно видеть, что $\zeta_{s}(\sigma)=\sum_{n} z_{n, s}^{-\sigma}$, где сумма взята по корням $f(z)$, расположенным внутри $C_{s}$ - таким образом, именно эта функция наиболее близка по структуре $\zeta$-функции оператора. В этом результате раскрыто различие между двумя типами $\zeta$-функций и одновременно показана их взаимосвязь, и, в частности, указана причина того, почему $\zeta$-функция, ассоциированная с функцией класса $K$, продолжается во всю плоскость как целая.

Далее в этих работах для одной серии корней были получены формулы регуляризованных следов. Заметим, что мероморфность соответствующей $\zeta$-функции приводит к весьма существенным проблемам в вычислении некоторых коэффициентов: появляется необходимость вычисления значений регулярной части $\zeta$-функции в точках полюсов. Обсудим, что означает суммирование одной серии корней с точки зрения теории ДО.

Обратим внимание на то, что обыкновенный ДО $n$-го порядка имеет решения с асимптотическим разложением по $\sqrt[n]{\lambda}$ и, соответственно, его характеристический определитель является функцией класса $K$ относительно переменной $\sqrt[n]{\lambda}$. Таким образом, его собственным числам соответствует $n$ серий корней соответствующей функции класса $K$, и суммирование одной серии корней $f(z)$ по существу означает суммирование дробных степеней собственных чисел оператора. Приведем две формулы для оператора Штурма-Лиувилля с условиями Дирихле. Пусть $q(x)$ вещественнозначная, все $\lambda_{n}>0$ и в формуле ниже 
$\sqrt{\lambda_{n}}>0$. Тогда [48]

$$
\begin{gathered}
\sum_{n=1}^{\infty}\left(\frac{1}{\sqrt{\lambda_{n}}}-\frac{1}{n}\right)=\int_{1}^{+\infty}\left(\sum_{n=1}^{\infty} \frac{1}{\lambda_{n}+t}-\frac{\pi}{2 \sqrt{t}}\right) \frac{d t}{\sqrt{t}}+\frac{2}{\pi} \sum_{n=1}^{\infty} \frac{1}{\sqrt{\lambda_{n}}} \operatorname{arctg} \frac{1}{\sqrt{\lambda_{n}}}-\gamma \\
\sum_{n=1}^{\infty}\left(\sqrt{\lambda_{n}}-n-\frac{1}{2 \pi n} \int_{0}^{\pi} q(x) d x+\frac{2}{\pi} \frac{1}{\sqrt{\lambda_{n}}} \operatorname{arctg} \frac{1}{\sqrt{\lambda_{n}}}-\frac{2}{\pi}\right) \\
=\frac{1}{12}-\frac{\gamma}{2 \pi} \int_{0}^{\pi} q(x) d x \\
+\int_{1}^{+\infty}\left(\sum_{n=1}^{\infty} \frac{1}{\lambda_{n}+t}-\frac{\pi}{2 \sqrt{t}}+\frac{1}{2 t}+\frac{1}{4 t \sqrt{t}} \int_{0}^{\pi} q(x) d x\right) \sqrt{t} d t
\end{gathered}
$$

здесь $\gamma-$ постоянная Эйлера.

В течение ряда лет применение и обобщение метода функций класса $K$ стало одним из центральных направлений развития теории. В работе [49] В. А. Садовничему удалось применить этот метод к ДО с неограниченным коэффициентом - краевой задаче для уравнения Бесселя

$$
-y^{\prime \prime}+\frac{\nu^{2}-1 / 4}{x^{2}} y+p(x) y=\lambda y
$$

на отрезке $[0, \pi]$, с краевым условием $y(\pi)=0$ (индекс дефекта этого оператора $(1,1))$, здесь $p(x) \equiv 0$ при $x \in[0, a], a<\pi, \nu \geqslant 1$. Собственные числа этого оператора - корни уравнения $y_{1}(\pi, \lambda)=0$, где $y_{1}(x, \lambda)$ - решение уравнения, принадлежащее $L_{2}[0, \pi]$. При $x \leqslant a$ решение выражается через функцию Бесселя: $y_{1}(x, \lambda)=\sqrt{x} I_{\nu}(\sqrt{\lambda} x) \lambda^{-\frac{\nu}{2}}$, и ее асимптотика хорошо известна, а при $x \in(a, \pi]$ асимптотика исследуется вполне стандартными методами, и получаем, что функция $y_{1}(\pi, \lambda)$ есть функция класса $K(18)$. Отметим, что даже при $p(x) \equiv 0$ в [49] получен нетривиальный результат, касающийся нулей $\lambda_{n, \nu}$ функции $I_{\nu}(\sqrt{\lambda} \pi)$. Например, формула первого следа в этом случае имеет вид

$8 \sum_{n=1}^{\infty}\left(\lambda_{n \nu}-\left(n+\frac{\nu}{2}-\frac{1}{4}\right)^{2}+\frac{1}{\pi^{2}}\left(\nu^{2}-\frac{1}{4}\right)\right)=\frac{\nu^{3}}{3}+\frac{\nu^{2}}{2}-\frac{\nu}{12}-\frac{1}{8}-\frac{\left(4 \nu^{2}-1\right)(\nu+1)}{\pi^{2}}$.

В связи с этим заметим, что суммы отрицательных степеней нулей функций Бесселя были предметом многочисленных исследований, некоторые суммы вычислили Дж. У. Рэлей и А. Кэли, и укажем в этой связи на работу П. Л. Капицы [50], в которой он нашел точные выражения сумм для всех отрицательных степеней, и на работу [51], содержащую некоторые обобщения.

Нетривиальное приложение и обобщение метода функций класса $K$ нашел В. А. Садовничий в работе [52]: для мероморфной функции $\Phi(z)=f_{2}(z) / f_{1}(z)$, где $f_{1}(z)$ и $f_{2}(z)$ - две функции класса $K(18)$, введена и изучена $\zeta$-функция

$$
Z_{0}(\sigma)=\frac{1}{2 \pi i} \int_{\Gamma_{0}} z^{-\sigma} \Phi(z) d z, \quad \operatorname{Re} \sigma>-1
$$

названная взвешенной $\zeta$-функцией, доказано, что $Z_{0}(\sigma)$ продолжается во всю плоскость как целая, при $\operatorname{Re} \sigma>-1$ получено представление сходящимся рядом

$$
Z_{0}(\sigma)=\sum_{n} \beta_{n} z_{1 n}^{-\sigma}
$$


где $z_{1 n}$ - нули $f_{1}(z)$, а $\beta_{n}$ - вычеты $\Phi(z)$, для всех достаточно больших номеров $\beta_{n}=f_{2}\left(z_{1 n}\right) / f_{1}^{\prime}\left(z_{1 n}\right)$. Далее в работе доказаны формулы регуляризованных следов и указан смысл этих формул в приложении к обыкновенным ДО: если в качестве функций $f_{1}(z)$ и $f_{2}(z)$ взять характеристические определители пары задач Штурма-Лиувилля, то $\beta_{n}=1 / \alpha_{n}$, где $\alpha_{n}=\int_{0}^{\pi} y_{n}^{2}(x) d x-$ так называемые нормировочные числа оператора, $y_{n}(x)$ - собственные функции, нормированные условием $y_{n}(0)=1$. Полученная формула следа нулевого порядка

$$
\sum_{n=1}^{\infty}\left(\frac{1}{\alpha_{1 n}}-\frac{1}{\alpha_{2 n}}\right)=0
$$

в терминах спектральной функции оператора $\rho(\lambda)=\sum_{\lambda_{n}<\lambda} \alpha_{n}^{-1}$ означает, что для любой пары операторов Штурма-Лиувилля с одним и тем же краевым условием в нуле имеет место асимптотическое равенство

$$
\rho_{1}(\lambda)=\rho_{2}(\lambda)+o(1), \quad \lambda \rightarrow \infty .
$$

Хорошо известно, сколь трудным был путь получения аналогичного результата для оператора на полуоси (см. [27]), и на этом фоне путь доказательства формулы (24) с помощью следов кажется совсем простым.

Еще одно обобщение метода Лидского-Садовничего было дано в цикле работ А. А. Стакуна (см. [53]), в которых были исследованы ДО второго порядка с точкой поворота. В указанном цикле работ для уравнения вида

$$
y^{\prime \prime}+\lambda^{2} q(x) y+R(x) y=0, \quad q(x)=x^{\alpha} r(x), \quad r(x)>0,
$$

были изучены различные граничные задачи на отрезке и полуоси, для различных вещественных значений $\alpha$, с гладкими коэффициентами. Особо были изучены случаи $\alpha=1$ и $\alpha=2$, рассмотрены задачи и с непрерывным спектром и с комплексным $R(x)$.

Цикл работ (см. [54] и приведенные там ссылки) А. С. Печенцова, основанный на методе Лидского-Садовничего, был посвящен исследованию асимптотического распределения собственных чисел и получению формул следов в случае, когда характеристический многочлен обыкновенного ДО имеет кратные корни. Такие задачи, как правило, выпадают из зоны прямого применения этого метода и требуют некоторой его модификации. При некоторых ограничениях здесь вместо функций класса $K(18)$ возникают целые функции вида

$$
\Delta(\lambda) \sim \sum_{k=1}^{H}\left(\exp \left(\sum_{j=0}^{h-1} \theta_{k j} \lambda^{\frac{h-j}{h}}\right) \lambda^{\frac{n_{k}}{h}} \sum_{\nu=0}^{\infty} \beta_{\nu}^{(k)} \lambda^{-\frac{\nu}{h}}\right) .
$$

Для построения $\zeta$-функции приходится выбирать контур интегрирования на римановой поверхности корня $\sqrt[h]{\lambda}$, и удается доказать аналоги основных свойств функции (19), что позволяет получить и формулы следа типа (21). Отметим, что для случая одного кратного корня характеристического многочлена А.С. Печенцов доказал, что структура асимптотических формул для корней рассматриваемых целых функций сохраняется такой же, как и в случае функций класса $K(18)$. 
Другое интересное приложение метода А. С. Печенцов нашел в одной сингулярной задаче (см. [55]), еще раз подтвердив, что приложения этого метода шире, чем некоторые классы регулярных задач. На полуоси для

$$
(-1)^{n} y^{(2 n)}+x y=\lambda y
$$

была рассмотрена краевая задача $y(0)=y^{\prime}(0)=\cdots=y^{(n-1)}(0)=0$ и найдены точные явные рекуррентные формулы для следов всех порядков.

Метод, основанный на исследовании корней целых функций, был применен в работе Ю. Белаббаси [56] для изучения многоточечной задачи

$$
\begin{gathered}
(-1)^{n} y^{(2 n)}+q(x) y=\lambda y, \quad y\left(\frac{j \pi}{2 n-1}\right)=0, \\
\int_{\frac{\pi j}{2 n-1}}^{\frac{\pi(j+1)}{2 n-1}} q(x) d x=0, \quad j=0, \ldots, 2 n-1 .
\end{gathered}
$$

В работе была найдена асимптотика собственных чисел и формула следа (' у знака суммы означает, что истинное число слагаемых взято с учетом дефекта регуляризации (22)):

$$
\sum_{k=1}^{\infty}\left(\lambda_{k}-\alpha k^{2 n}\right)=-\frac{2 n-1}{4 n} \sum_{j=0}^{2 n-1} q\left(\frac{\pi j}{2 n-1}\right)
$$

здесь $\alpha$ - известное число. Несколько ранее им же была получена формула следа в многоточечной задаче для оператора четвертого порядка без ограничительных условий на интегралы от потенциала.

Метод функций класса $K$ оказался применим и к исследованию дифференциально-функциональных задач. В работе М. Мартиновича [57] рассмотрена краевая задача $y(0)=y(\pi)=0$ для уравнения

$$
-y^{\prime \prime}+q(x) y=\lambda y+\sum_{k=1}^{n-1} \alpha_{k} y\left(\frac{k \pi}{n}\right)+\alpha_{n} \int_{0}^{\pi} y(t) d t
$$

и исследованы асимптотические свойства решений уравнения, собственных чисел задачи, изучено аналитическое продолжение $\zeta$-функции оператора и доказаны формулы следов всех порядков. Формула первого следа для случая $n=2$, $\alpha_{1}=1, \alpha_{2}=0$ и потенциала с нулевым средним имеет вид

$$
\sum_{n=0}^{\infty} \sum_{j=1}^{4}\left(\lambda_{n j}-(4 n+j)^{2}-\frac{c_{j}}{4 n+j}\right)=-\frac{q(\pi)+q(0)}{4},
$$

где $c_{1}=4 / \pi, c_{3}=-4 / \pi, c_{2}=c_{4}=0$.

Метод работы [52] исследования взвешенной $\zeta$-функции был применен в ряде работ 3. Каделбургом. Два приложения к известным (существенно несамосопряженным) задачам математической физики содержится в [58]. В задаче Редже $(q(x)$ - комплекснозначная)

$$
-y^{\prime \prime}+q(x) y=s^{2} y, \quad y(0)=y^{\prime}(a)+i s y(a)=0
$$


$q(x) \sim c_{\mu}(a-x)^{\mu}$ при $x \rightarrow a-0, \mu \geqslant 0, c_{\mu} \neq 0$, автор определил нормировочные константы и обобщенную спектральную функцию (см. [27]) равенствами

$$
\alpha_{n}=\int_{0}^{a} \varphi_{n}^{2}(x) d x-\frac{i \varphi_{n}^{2}(a)}{2 s_{n}}, \quad \rho(\lambda)=\sum_{\left|\operatorname{Re} s_{n}\right|<\lambda} \alpha_{n}^{-1}
$$

и доказал следующую формулу взвешенного следа нулевого порядка:

$$
\sum_{n=1}^{\infty}\left(\frac{1}{\alpha_{n}}-b n^{2}+c \ln ^{2} n+d \ln n+e\right)=-\frac{q^{\prime}(0)}{4}-b \zeta(-2)+c \zeta^{\prime \prime}(0)-d \zeta^{\prime}(0)+e \zeta(0),
$$

где

$$
\begin{gathered}
b=2 \pi^{2} / a^{3}, \quad c=(\mu+2)^{2} /\left(2 a^{3}\right), \quad d=(\mu+2)(\ln A+\mu+2) / a^{3}, \\
e=\left(\ln ^{2} A+2(\mu+2) \ln A-2 a B+2 a^{2} q(0)\right) /\left(2 a^{3}\right), \\
A=-(2 \pi i)^{\mu+2} /\left(a^{\mu+2} q^{(\mu)}(a)\right), \quad B=\int_{0}^{a} q(x) d x-q^{(\mu+1)}(a) /\left(2 q^{(\mu)}(a)\right) .
\end{gathered}
$$

Также и для задачи Орра-Зоммерфельда З. Каделбург [58] определил обобщенную спектральную функцию и получил след.

В работе [59] 3. Каделбург для самосопряженной задачи, заданной двучленным ДО, доказал формулу $\sum_{n=1}^{\infty}\left(\pi \alpha_{n}^{-1}-2 k n^{k-1}\right)=-2 k \zeta(1-k)$, из которой следует совпадение спектральных функций таких операторов с точностью до бесконечно малой при $\lambda \rightarrow \infty$ величины (ср. с (24)). Наконец, в работе [60] Каделбурга и Мартиновича исследованы следы с весом для некоторого семейства дифференциально-функциональных уравнений.

Хотя изначально теория функций класса $K$ была написана с идеей применения к ДО с достаточно гладкими коэффициентами, ее оказалось возможным применять и для исследования операторов с кусочно-гладкими коэффициентами. В цикле работ С. И. Митрохина (см. [61] и там дальнейшие ссылки) были исследованы такие задачи. Так, например, он исследовал задачу

$$
-k(x) y^{\prime \prime}+q(x) y=\lambda y, \quad y(0)=y(\pi)=0
$$

с $k(x)=a_{j}^{2}, q(x)=a_{j}^{2} q_{j}(x), j$ - номер интервала, на которые разбит отрезок: $0=x_{0}<x_{1}<\cdots<x_{N}<x_{N+1}=\pi$, и с условиями склейки

$$
y_{j+1}\left(x_{j}+0\right)=y_{j}\left(x_{j}-0\right), \quad a_{j+1} y_{j+1}^{\prime}\left(x_{j}+0\right)=a_{j} y_{j}^{\prime}\left(x_{j}-0\right) .
$$

Были получены формулы следа любого порядка (порядок зависит от гладкости функций $\left.q_{j}(x)\right)$, формула первого следа имеет вид

$$
\begin{aligned}
& \sum_{n=1}^{\infty}\left(\lambda_{n}-\frac{\pi^{2} n^{2}}{m^{2}}-\frac{2 \pi c}{m}\right)=-\frac{1}{4}(q(0)+q(\pi))+\frac{\pi c}{m} \\
& \quad+\frac{2}{\pi} \sum_{j=1}^{N}\left(a_{j+1}^{2} q\left(x_{j}+0\right)-a_{j}^{2} q\left(x_{j}-0\right)\right) \sum_{n=1}^{\infty} \frac{1}{n} \sin \frac{2 \pi n x_{j} a_{j+1}}{\pi a_{j}+x_{j}\left(a_{j+1}-a_{j}\right)},
\end{aligned}
$$


где

$$
c=\frac{1}{2 \pi} \sum_{j=1}^{N=1} a_{j} \int_{x_{j-1}}^{x_{j}} q(t) d t, \quad m=\sum_{j=1}^{N+1} \frac{\pi a_{j}+x_{j}\left(a_{j+1} a_{j}\right)}{a_{j} a_{j+1}} .
$$

Также С. И. Митрохин исследовал дифференциально-функциональные операторы с разрывными коэффициентами и оператор Штурма-Лиувилля с разрывной весовой функцией. Во всех случаях были доказаны формулы следов и явно предъявлены формулы первого следа.

Оказалось возможным применить метод функций класса $K$ и к задачам с запаздывающим аргументом. М. Пикула в работе [62] изучил задачу второго порядка:

$$
\begin{gathered}
-y^{\prime \prime}+q(x) y(x-\tau)=\lambda y, \quad y^{\prime}(0)-h y(0)=y^{\prime}(\pi)+H y(\pi)=0, \\
y(x-\tau)=y(0) \varphi(x-\tau) \quad \text { при } \quad x \leqslant \tau, \quad \varphi(0)=1 .
\end{gathered}
$$

Интересно сравнить две формулы первого следа: при $\tau \geqslant \pi$

$$
\sum_{n=1}^{\infty}\left(\lambda_{n}(\tau)-n^{2}\right)=q(0) \varphi(-\tau)-\frac{h^{2}+H^{2}}{2},
$$

а при $\tau<\pi$

$$
\begin{aligned}
& \sum_{n=1}^{\infty}\left(\lambda_{n}(\tau)-n^{2}-\frac{2}{\pi}\left(h+H+\frac{\cos n \tau}{2} \int_{\tau}^{\pi} q(t) d t\right)\right) \\
& \quad=q(0) \varphi(-\tau)-\frac{h^{2}+H^{2}}{2}+\frac{h+H}{\pi}-\frac{1}{2 \pi} \int_{\tau}^{\pi} q(t) d t+\frac{(q(\pi)-3 q(\tau))(\pi-\tau)}{4 \pi} .
\end{aligned}
$$

Эти соотношения можно (весьма косвенно, конечно) трактовать как формулы обратной задачи, похожие соотношения активно исследовались в периодическом случае, о котором речь пойдет ниже.

Хотя метод функций класса $K$ оказался применим к весьма широкому кругу задач и доказал свою эффективность, ограничения по его применимости к обыкновенным ДО вызывали неудовлетворенность и требовали дальнейших исследований. В первую очередь это относилось к требованию соизмеримости длин отрезков, на которые показатели $\alpha_{k}$ делят стороны своей выпуклой оболочки (18). Один из возможных путей распространения теории на более широкий класс функций был предложен в работах В. А. Садовничего, В. А. Любишкина и Ю. Белаббаси (см. [63]). Здесь был рассмотрен случай, когда показатели $\alpha_{j}$ (см. (18)) делят некоторые отрезки своей выпуклой оболочки на несоизмеримые части. Для каждого отрезка выпуклой оболочки по точкам $P_{j}\left(\alpha_{j}, n_{j}\right)$ (здесь координаты точки - параметры асимптотики (18)) строится ломаная Ньютона, и во всех случаях, кроме существования в этой ломаной отрезков, параллельных действительной оси и делимых точками $P_{j}$ на несоизмеримые части, удалось получить все результаты, аналогичные [11], - аналитическое продолжение $\zeta$-функции, асимптотическое распределение собственных чисел и формулы следов всех порядков. Отметим, что роль $\zeta$-функции Римана в этой задаче играла известная специальная функция $F(a, \lambda)=\sum_{n=1}^{\infty} a^{n} n^{-\lambda}$, которая при значении параметра $a=1$ является как раз $\zeta$-функцией Римана. 
Дальнейшее обобщение было получено В. А. Садовничим и В. А. Любишкиным в статье [64]. В этой работе для класса $C$ целых функций экспоненциального типа $\sigma$, характеризуемого следующими свойствами:

1) все корни функции $f(z)$ лежат в горизонтальной полосе $|\operatorname{Im} z|<h$;

2) при некотором фиксированном значении $\operatorname{Im} z$ верны неравенства

$$
0<m \leqslant|f(\operatorname{Re} z+i \operatorname{Im} z)| \leqslant M<\infty
$$

3) типы функции $f(z)$ в верхней и нижней полуплоскостях совпадают, исследована ассоциированная $\zeta$-функция и предложен метод вычисления регуляризованных сумм корней функции $f_{1}(z)$, имеющей представление вида

$$
f_{1}(z)=f(z)+z^{-1} A_{1}(z)+\cdots+z^{-m} A_{m}(z)+O\left(z^{-m-1} \exp (\sigma|\operatorname{Im} z|)\right)
$$

при $z \rightarrow \infty$, здесь $f(z)$ - функция класса $C, A_{k}(z)$ - целые функции, допускающие оценку $\left|A_{k}(z)\right| \leqslant M_{1} \exp (\sigma|\operatorname{Im} z|)$. Подчеркнем, что сама функция $f_{1}(z)$ может не принадлежать классу $C$. Корни функций класса $C$ расположены сложно, и формулы следов удалось получить только с расстановкой скобок.

Важным представителем класса $C$ явились функции класса $K$ с несоизмеримостью отрезков на выпуклой оболочке показателей, и в работе С. А. Салеха [65] метод функций класса $C$ был применен к многоточечной задаче

$$
\begin{gathered}
-y^{\prime \prime}+q(x) y=\lambda y, \\
\alpha_{1} y(0)+\alpha_{2} y(\pi)+\sum_{k=3}^{n} \alpha_{k} y\left(\pi x_{k}\right)=\beta_{1} y(0)+\beta_{2} y(\pi)+\sum_{k=3}^{n} \beta_{k} y\left(\pi x_{k}\right)=0,
\end{gathered}
$$

где $\alpha_{1} \beta_{2}-\alpha_{2} \beta_{1} \neq 0,0<x_{3}<\cdots<x_{n}<1$. Были получены формулы следов без дополнительных условий на $x_{k}$, что невозможно применением метода функций класса $K$. Также здесь рассмотрен пример с краевым условием, содержащим интегральное слагаемое.

Еще Л. А. Дикий (см. [34] и там дальнейшие ссылки) рассматривал задачу о регуляризации рядов, содержащих собственные функции оператора, наиболее важная и естественная постановка - суммирование интегрального ядра семейства спектральных проекторов оператора. Весьма общие результаты в этом направлении, основанные на методе Лидского-Садовничего, были получены в работах Г. С. Типенко (см. [66]). Автор разработал метод суммирования вычетов мероморфных функций вида $H(x, \xi, z) / \Delta(z)$ с параметрами $x$ и $\xi$, где $H(x, \xi, z)$ и $\Delta(z)$ - целые функции экспоненциального типа с некоторыми дополнительными условиями, и применил его для исследования задачи с весьма общим видом краевых условий и сложным вхождением спектрального параметра

$$
\begin{gathered}
y^{(m)}+p_{1}(x, z) y^{(m-1)}+\cdots+p_{m}(x, z) y=0, \\
U_{i}(y)+\sum_{k=0}^{\varkappa_{i}} \int_{0}^{1} y^{(k)} d \sigma_{i k}(x)=0,
\end{gathered}
$$

где $\varkappa_{i} \leqslant m-1, \quad i=1, \ldots, m, U_{i}(y)$ - двухточечные функционалы, образующие обычные регулярные нераспадающиеся краевые условия, $p_{k}(x, z)=$ $\sum_{n=0}^{k} p_{k n}(x) z^{n}, \quad k=1, \ldots, m, \sigma_{i k}(x)$ - функции ограниченной вариации на 
отрезке $[0,1]$, непрерывные в точках 0 и 1 , корни характеристического уравнения $\lambda^{m}+p_{11}(x) \lambda^{m-1}+\cdots+p_{m m}(x)=0$ различны, отделены от нуля и их аргументы и аргументы их разностей не зависят от $x$. Г. С. Типенко получил общие формулы регуляризованных сумм собственных функций; например, для оператора Штурма-Лиувилля (3) приведем формулу

$$
\begin{array}{r}
\sum_{n=1}^{\infty}\left(y_{n}(x) y_{n}(\xi)-2 \sin \pi n x \sin \pi n \xi+\frac{1}{\pi n}(q(x) \cos \pi n x \sin \pi n \xi\right. \\
\quad-q(1-\xi) \cos \pi n \xi \sin \pi n x))=\frac{1}{2}((1-\xi) q(x)+x q(1-\xi))
\end{array}
$$

здесь $y_{n}(x)$ - нормированные собственные функции оператора, $x \leqslant \xi, q(x)=$ $\int_{0}^{x} p(t) d t, \int_{0}^{1} p(t) d t=0, p(t)$ - потенциал оператора.

Аналогичную задачу рассмотрел Ю. В. Матвеев [67] для дифференциальнофункциональной задачи

$$
y^{(n)}+p_{2}(x) y^{(n-2)}+\cdots+\left(p_{n}(x)-\rho^{n}\right) y=A(x) \sum_{i=1}^{s_{1}} \rho^{q_{i}} F_{i}(y)
$$

с регулярными краевыми условиями, где $F_{i}(y)=A_{1 i} \int_{x_{1 i}}^{x_{2 i}} A_{i}(\xi) y(\xi) d \xi$ при $i=$ $1, \ldots, s$ и $F_{i}(y)=A_{1 i} y^{\left(n_{i}\right)}\left(x_{i}\right)$ при $i=s+1, \ldots, s_{1}, 0<x_{i}<\pi, 0 \leqslant x_{1 i}<x_{2 i} \leqslant \pi$, $0 \leqslant n_{i} \leqslant n-1$, еще ряд условий, несущественных для общего восприятия задачи, мы не приводим. Автор получил ряд формул сумм собственных функций, в том числе регуляризованные суммы значений собственных функций в одной точке.

После работ [12], [13] изучение формул следов в задачах рассеяния было интенсивно продолжено. Крупное продвижение было сделано в работах В. С. Буслаева [68], [69], в которых формулы следов были получены для оператора Шрёдингера

$$
H(u)=-\Delta u+q(x) u
$$

в трехмерном пространстве, здесь $q(x) \in C^{\infty}$ равномерно убывает на бесконечности вместе со всеми своими производными быстрее любой степени $|x|^{-1}$. Автор нашел равномерное по $\arg \lambda$ асимптотическое разложение интегрального ядра резольвенты и получил при $\lambda \rightarrow \infty$ асимптотику

$$
\operatorname{Tr}\left(R_{\lambda}-R_{\lambda}^{0}\right) \sim \sum_{n=1}^{\infty}(-1)^{n}\left(\frac{1}{2 i \sqrt{\lambda}}\right)^{n} \frac{1}{4 \pi} \int_{\mathbb{R}^{3}} \Omega_{n}^{(1)}(x, x) d x
$$

(функции $\Omega_{n}^{(1)}(x, x)$ можно найти из рекуррентных соотношений) и равенство

$$
\operatorname{Tr}\left(R_{\lambda}-R_{\lambda}^{0}\right)=\int_{-\infty}^{0} \frac{d N_{-}(\mu)}{\mu-\lambda}+\frac{1}{2 \pi i} \int_{0}^{+\infty} \frac{d}{d \mu}(\ln \operatorname{det} S(\mu)) \frac{d \mu}{\mu-\lambda},
$$

где $N_{-}(\mu)$ - считающая функция отрицательного спектра, $S(\mu)$ - матрица рассеяния. Из сравнения этих выражений были получены формулы следа всех 
порядков, первый след имеет вид

$$
\sum_{l=1}^{M} \lambda_{l}=\frac{1}{2 \pi i} \int_{0}^{\infty}\left(\ln \operatorname{det} S(\lambda)+\frac{2 i \sqrt{\lambda}}{4 \pi} \int_{\mathbb{R}^{3}} q(x) d x+\frac{1}{8 \pi i \sqrt{\lambda}} \int_{\mathbb{R}^{3}} q^{2}(x) d x\right) d \lambda
$$

Также в работе для функции спектрального сдвига рассматриваемой пары операторов было получено точное выражение, подобное (15). В статье [70] аналогичное исследование было проведено для задачи рассеяния во внешности области. Отметим еще, что в статье [69] имеется краткий обзор некоторых малоизвестных работ, не вошедших в наш список литературы, в том числе содержащих физические приложения.

Совсем недавно формулы следов всех порядков типа (13) и (14) были получены в работах А. В. Рыбкина [71] и С. М. Белова и А. В. Рыбкина [72] для дальнодействующих, т.е. не имеющих конечного первого момента, потенциалов.

Ряд авторов - Ф. А. Березин, В. С. Буслаев, С. П. Меркурьев [73]-[75] применили формулы следов в задачах рассеяния для вывода новых формул для второго и третьего групповых интегралов некоторых задач статистической физики. Выделим здесь работу В. С. Буслаева [74], в которой помимо изложения собственных результатов автор дает критический анализ ряда работ других авторов и указывает на связь этих исследований с классической формулой статистической физики Бете-Уленбека [76]. Одновременно велись и очень близкие по сути исследования формул следов в модели Фридрихса, укажем здесь на работу Д. Р. Яфаева [77], основной результат которой мы приведем для иллюстрации. В пространстве $L_{2}\left(\mathbb{R}^{2} ; N\right)$ вектор-функций $f\left(x_{1}, x_{2}\right)$ со значениями во вспомогательном гильбертовом пространстве $N$ рассматриваются операторы: $H_{0}$ - оператор умножения на $x_{1}+x_{2}, V_{j}$ - линейные операторы с ядром (в смысле теоремы Л. Шварца) вида $q_{j}\left(x_{j}, x_{j}^{\prime}\right) \delta\left(x_{i}-x_{i}^{\prime}\right), j=1,2, i \neq j$, $\delta(\cdot)$ - дельта-функция, $H=H_{0}+V_{1}+V_{2}, H_{j}=H_{0}+V_{j}$. Предполагается, что $q_{j}\left(x_{j}, x_{j}^{\prime}\right)$ задают ядерные операторы в $N, q_{j}^{*}\left(x, x^{\prime}\right)=q_{j}\left(x^{\prime}, x\right)$ и что в ядерной норме функции $q_{j}\left(x_{j}, x_{j}^{\prime}\right)$ обладают достаточной гладкостью и быстро убывают на бесконечности. Одновременно рассматриваются в пространстве $L_{2}(\mathbb{R} ; N)$ операторы: $h_{0}$ - оператор умножения на $x, v_{j}$ - линейные операторы с ядром $q_{j}\left(x, x^{\prime}\right), j=1,2, h_{j}=h_{0}+v_{j}$. Через $R(z), R_{j}(z), R_{0}(z)$ и $r_{j}(z)$ обозначаются резольвенты операторов $H, H_{j}, H_{0}$ и $h_{j}, R_{c}=R-R_{0}-\left(R_{1}-R_{0}\right)-\left(R_{2}-R_{0}\right)$, a $S(E), S_{j}(E), s_{j}(E)$ - матрицы рассеяния для пар $\left(H_{0}, H\right),\left(H_{0}, H_{j}\right),\left(h_{0}, h_{j}\right)$. Тогда формула следа работы [77] имеет вид

$$
\begin{aligned}
2 i \operatorname{Im} \operatorname{Tr} R_{c}(E+i 0)=\operatorname{Tr} & \left(S^{*}(E) S^{\prime}(E)-S_{1}^{*}(E) S_{1}^{\prime}(E)-S_{2}^{*}(E) S_{2}^{\prime}(E)\right) \\
& +\frac{1}{4 \pi i} \sum_{i \neq j} \int_{-\infty}^{+\infty} \operatorname{Tr}_{N}\left(s_{i}^{*}(\lambda) s_{i}^{\prime}(\lambda) s_{j}^{\prime}(E-\lambda) s_{j}^{*}(E-\lambda)\right) d \lambda .
\end{aligned}
$$

Ряд авторов использовали формулы регуляризованных следов для исследования распределения резонансных состояний в задаче рассеяния, из последних работ укажем на статью С. А. Степина [78], в которой можно найти дальнейшие ссылки. Отметим в этом контексте и более раннюю работу А. В. Рыбкина [79], получившего некоторую формулу следов для резонансов. 
На ранней стадии развития теории периодические задачи почти не рассматривались: для ДО на отрезке существенным барьером был переход от распадающихся краевых условий к нераспадающимся, для операторов на прямой с непрерывным спектром более чем существенной трудностью было неубывание потенциала. После уже упоминавшейся первой работы Ч. Хальберга и В. Крамера [36] дальнейшее продвижение было сделано в работе Г. Хохштадта [80], причем здесь впервые были соединены исследования регулярной и сингулярной задач (см. ниже формулу (29)). Конечно, метод функций класса $K$ полностью решал вопрос для задач на отрезке, но в связи с приложениями в периодической задаче были особые требования к алгоритму вычислений и форме ответа, а также особый интерес к формулам, содержащим собственные числа двух (а иногда и большего числа) заметно различающихся задач, а не к формулам с регуляризацией степенями натуральных чисел. Все эти задачи можно решить методом функций класса $K$, но никем это сделано не было.

По настоящему пристальный и детальный интерес к периодическому случаю возник после открытия метода обратной задачи в интегрировании ряда классических нелинейных уравнений математической физики. Интерпретация этих уравнений как гамильтоновых систем с бесконечным числом степеней свободы привела к открытию у них бесконечной системы сохраняющихся (независящих от времени) величин - первых интегралов, или законов сохранения. Оказалось, что следы операторов с непрерывным спектором, в частности, формулы следов оператора Штурма-Лиувилля, найденные в работах Л. Д. Фаддеева и В. С. Буслаева [12], [13], дают законы сохранения для динамической системы, описываемой уравнением Кортевега-де Фриза. Первой работой, в которой была дана такая интерпретация, стала работа В. А. Захарова и Л. Д. Фаддеева [14]. В этот период вышло несколько работ, в которых разрабатывались различные методы вычисления формул следов в задачах рассеяния в связи с методом обратной задачи интегрирования нелинейных эволюционных уравнений. Мы безусловно выделим работу И. М. Гельфанда и Л.А. Дикого [81], в которой, во-первых, была вскрыта алгебраическая сторона вопроса, а во-вторых, применены два метода вычисления формул следов, прямо восходящих к работам этих же авторов по следам оператора Штурма-Лиувилля: метод, основанный на асимптотическом разложении резольвенты оператора, и метод, связанный с исследованием ל-функции. В работе Б. М. Левитана [82] волновое уравнение решено двумя способами: методом Римана и методом Фурье, результаты приравнены и полученное равенство проварьировано по потенциалу, из чего и найдены плотности интегралов движения для уравнения Кортевега-де Фриза. Подробнее об этой теории написано в ряде обзоров и монографий (см., например, [83]), из современных работ выделим статью Ф. Гештези и Х. Холдена [84] и работу А.В. Рыбкина [85], посвященные прямо нашей основной теме (в них можно найти дальнейшие ссылки). Также укажем, что сравнительно недавно [86] формулы следов типа (14) были применены для исследования вопроса о структуре спектра операторов с потенциалом из $L_{2}$.

Но особый интерес в это время вызвала задача о вычислении различных форм формул регуляризованных следов для задач на конечном отрезке с периодическими и антипериодическими краевыми условиями и периодической задачи на всей прямой. Это было связано как с важностью периодических 
задач в физических приложениях (что проявилось в особом интересе к этому случаю при исследовании нелинейных уравнений методом обратной задачи), так и с наличием прямой связи дискретного спектра задач на отрезке с непрерывным спектром задач на прямой.

Среди большого количества работ, содержащих исследования этой задачи, мы выделим фундаментальную работу Г. Маккина и П. ван Мёрбеке [87], посвященную спектральной теории оператора Хилла, в которой был предложен оригинальный и изящный метод получения формул следов. В пространстве $L_{2}(-\infty,+\infty)$ рассматривается оператор

$$
-\frac{d^{2}}{d x^{2}}+q(x)
$$

где функция $q(x) \in C^{\infty}(-\infty,+\infty)$ и периодическая с периодом 1 , через $\lambda_{n}$ обозначим границы интервалов спектра этого оператора:

$$
\left[\lambda_{0}, \lambda_{1}\right] \cup\left[\lambda_{2}, \lambda_{3}\right] \cup\left[\lambda_{4}, \lambda_{5}\right] \cup \cdots, \quad-\infty<\lambda_{0}<\lambda_{1} \leqslant \lambda_{2}<\lambda_{3} \leqslant \lambda_{4}<\cdots,
$$

через $\mu_{n}$ - спектр задачи Дирихле для оператора на отрезке $[0,1]$ с дифференциальным выражением (26). Хорошо известно, что $\mu_{i} \in\left[\lambda_{2 i-1}, \lambda_{2 i}\right], i=1,2, \ldots$. В работе была найдена асимптотика $\theta$-функции при $t \rightarrow+0$

$$
\sum_{n=0}^{\infty} e^{-\lambda_{n} t} \sim \frac{1}{\sqrt{\pi t}}+\frac{1}{\sqrt{\pi t}} \sum_{m=1}^{\infty} \frac{(-t)^{m} H_{m-1}}{1 \cdot 3 \cdots(2 m-3)}
$$

где $H_{m}=\int_{0}^{1} I_{m}\left(q(x), q^{\prime}(x), \ldots\right) d x, I_{m}$ - полином, и доказаны формулы следов

$$
\lambda_{0}^{p}+\sum_{i=1}^{\infty}\left(\lambda_{2 i-1}^{p}+\lambda_{2 i}^{p}-2 \mu_{i}^{p}\right)=S_{p}
$$

где числа $S_{p}$ определяются из равенства степенных рядов

$$
\sum_{p=1}^{\infty}(2 \varepsilon)^{p} S_{p} / p=2 \ln \left(\sum_{j=0}^{\infty} \varepsilon^{j} \frac{\partial H_{j}(0)}{\partial q}\right)
$$

Приведем несколько первых ответов:

$S_{1}=q(0), \quad S_{2}=q^{2}(0)-\frac{1}{2} q^{\prime \prime}(0), \quad S_{3}=q^{3}(0)-\frac{15}{16} q^{\prime}(0)-\frac{3}{2} q(0) q^{\prime \prime}(0)+\frac{3}{16} q^{(4)}(0)$.

Также в [87] с помощью оригинального технического приема были найдены формулы и другого канонического вида, прямо восходящего к пионерским работам [7], [26], [34]. Из предыдущих формул следует, что при $t \rightarrow+0$ верно разложение

$$
\sum_{n=1}^{\infty} e^{-\mu_{n} t} \sim \frac{1}{\sqrt{4 \pi t}}+\frac{1}{\sqrt{4 \pi t}} \sum_{m=1}^{\infty} \frac{(-t)^{m} H_{m-1}}{1 \cdot 3 \cdots(2 m-3)}-\frac{1}{2} \sum_{p=0}^{\infty} \frac{(-t)^{p}}{p !} S_{p}
$$


и, заменяя (с точностью до $\left.O\left(t^{-\infty}\right)\right) 1 / \sqrt{4 \pi t}$ на $1 / 2+\sum_{l=1}^{\infty} \exp \left(-l^{2} \pi^{2} t\right)$ с последующим разложением по степеням $t$ уже рядов из экспонент, получаем

$$
2 \sum_{l=1}^{\infty}\left(\mu_{l}^{p}-\sum_{i+j=p} \frac{p !\left(l^{2} \pi^{2}\right)^{i} H_{j-1}}{1 \cdot 3 \cdots(2 m-3) i !}\right)=\frac{p ! H_{p-1}}{1 \cdot 3 \cdots(2 p-3)}-S_{p} .
$$

Отметим, что первым формулу типа (28) получил Г. Хохштадт [80] в случае, когда в спектре периодической задачи есть одна и только одна лакуна, и применил ее для решения обратной задачи. Он рассмотрел семейство операторов на отрезке в периодической задаче, потенциалы которых отличались лишь сдвигом на параметр $\tau$, и получил формулу

$$
\mu(\tau)-\mu(0)=-\frac{1}{2}(q(\tau)-q(0))
$$

где $\mu$ - первое собственное значение задачи с закрепленными концами (оно лежит внутри единственной лакуны в спектре оператора Хилла).

Мы еще будем встречать обобщения формулы (29) именно как своеобразного инструмента обратной задачи, например, из этого результата автор получил, что единственная лакуна в спектре может быть, только если потенциал есть эллиптическая функция. В той же работе была получена и формула следа в случае конечного числа лакун $\sum_{i=1}^{n}\left(\mu_{i}(\tau)-\mu_{i}(0)\right)=-\frac{1}{2}(q(\tau)-q(0))$, но получить аналогичное следствие из нее не удалось, развитие эта тема получила в работе [87] и вышедшей почти одновременно работе [88] (с другой техникой получения формул следов). Позже аналогичные формулы следов удалось получить (и применить к обратной задаче) и в почти периодическом случае, см., например [89], и для еще более широких классов операторов [90]. Мы далее в эту тему углубляться не будем, ограничившись заключительным упоминанием работы Б. М. Левитана [91], в которой методами теории следов для регулярного оператора Штурма-Лиувилля с периодическими краевыми условиями был изучен примыкающий к обратной задаче вопрос об условиях на спектр, обеспечивающих гладкую периодичность потенциала. Попутно был предложен новый метод вычисления взвешенных регуляризованных следов с помощью функции Римана задачи Коши соответствующего волнового уравнения, основанный на аналогичном методе получения спектральных формул следов работ [82], [92]. Отметим полученное в этой работе утверждение о том, что для бесконечно дифференцируемого потенциала все регуляризованные следы антипериодической задачи равны нулю.

\section{Регуляризованные следы. Общая теория}

Все обсуждавшиеся нами задачи не охватывали ДО в частных производных с дискретным спектром. Причин здесь несколько: методы, связанные с наличием у обыкновенных уравнений фундаментальной системы решений, не могли в этих задачах работать в принципе; методы, основанные на использовании асимптотических разложений резольвенты или $\theta$-функции, либо на аналитическом продолжении $\zeta$-функции, требовали существования у собственных чисел точной асимптотики с остаточным членом, по меньшей мере образующим сходящийся ряд, что также не имеет места у операторов в частных производных. 
В этой ситуации на первый план стали выходить задачи для абстрактных операторов - вопрос ставился следующим образом: каким требованиям должны удовлетворять дискретный оператор $A$ и подчиненный ему оператор $B$, с тем чтобы начальный отрезок ряда теории возмущений для собственных чисел оператора $A+B$ являлся регуляризацией этих собственных чисел, достаточной для сходимости регуляризованного следа, и какова точно длина этого отрезка? Связь этого вопроса с фундаментальной идеей расширения понятия следа оказалась наиболее близкой среди всех вариантов постановки задачи, о чем мы уже говорили при обсуждении соотношения (6), но нельзя упускать из виду самостоятельную ценность этой задачи и прямую связь с теорией возмущений дискретного спектра, которая делает всякий результат этого раздела теории следов также и новым результатом теории возмущений.

Длительное время после работ [8], [36], [9] исследований в этом направлении не велось, и только в 1977 году работа В. А. Садовничего и В. В. Дубровского [15] вновь вернула интерес к этим задачам. В этой работе были доказаны формулы регуляризованных следов всех порядков при возмущении самосопряженного дискретного полуограниченного оператора $T$ оператором $P$, являющимся относительно ядерным для любой отрицательной степени оператора $T$, и в качестве примера было рассмотрено возмущение задачи Дирихле для оператора Лапласа $-\Delta$ в прямоугольном параллелепипеде П с соизмеримыми ребрами ИО с финитным ядром $K(x, y) \in C^{\infty}(\Pi)$.

Вскоре вышла вторая работа этих авторов [16], в которой была усилена абстрактная теорема о следе, что позволило в качестве примера впервые рассмотреть ДО, а именно, формула следа была получена для возмущения оператором умножения на функцию $p(x, y)$ степени $3+\varepsilon$ оператора Лапласа на двумерном прямоугольнике с условиями Дирихле, и при некоторых ограничениях на потенциал формула приобретает вид, весьма сходный с формулой Гельфанда-Левитана:

$$
\sum\left(\lambda_{n}-\mu_{n}\right)=-\frac{p(0,0)+p(a, 0)+p(0, b)+p(a, b)}{16}
$$

В этой же работе формула следа была доказана и для билапласиана, но для потенциалов, ряд Фурье которых содержит конечное число ненулевых слагаемых. Далее эти результаты уточнялись в работах В. А. Садовничего и его учеников (см. литературу в работах [93], [94]), основной идеей во всех было интегрирование разности резольвент по системе расширяющихся контуров, основным инструментом усиления результатов - уточнение оценки резольвент. Мы ниже обсудим более поздние значительные продвижения на этом направлении, и там подробнее обсудим идеи и технику.

Исследование формул следов в случае конечномерного возмущения было проведено в статье В. А. Садовничего и В. А. Любишкина [93]. Конечномерные возмущения являются едва ли не важнейшим классом возмущений, многие задачи теории операторов к ним сводятся, в том числе пионерские работы по следам [3], [4] основаны на исследовании конечномерных возмущений. В обсуждаемой работе рассматривалось возмущение самосопряженного дискретно- 
го оператора $A$ конечномерным оператором

$$
B u=\sum_{k=1}^{r}\left(A u, f_{k}\right) g_{k}
$$

где $f_{k}$ и $g_{k}$ - некоторые векторы пространства. Подчеркнем, что так как векторы $f_{k}$, вообще говоря, не принадлежат области определения оператора $A$, то оператор $B$ может быть неограничен. Основной результат работы заключается в доказательстве для оператора $A$, асимптотика считающей функции которого имеет вид

$$
N(\lambda)=c \lambda+O\left(\lambda^{p}\right), \quad c \geqslant 0, \quad 0<p<1
$$

формулы

$$
\lim _{n \rightarrow \infty} \sum_{k=1}^{k_{n}}\left(\mu_{k}-\lambda_{k}\right)=\sum_{j=1}^{r}\left(A^{1-q_{j}} g_{j}, A^{q_{j}} f_{j}\right),
$$

где $0 \leqslant q_{j} \leqslant 2, f_{j} \in \mathscr{D}\left(A^{q_{j}}\right), g_{j} \in \mathscr{D}\left(A^{2-q_{j}}\right)$. Особым достижением являлось включение в формулировку случая $c \neq 0$ в асимптотике $N(\lambda)$. Приведем два примера из этой работы. Первый пример - это возмущение регулярного обыкновенного ДО $A$ одномерным оператором

$$
B u=h(x) \int_{a}^{b} G\left(x_{0}, \xi\right) A u(\xi) d \xi=u\left(x_{0}\right) h(x),
$$

где $G(x, \xi)$ - функция Грина оператора $A, h(x) \in \mathscr{D}\left(A^{2}\right)$, и здесь (31) дает формулу следа

$$
\sum_{n=1}^{\infty}\left(\mu_{n}-\lambda_{n}\right)=h\left(x_{0}\right) .
$$

Второй пример - возмущение самосопряженного эллиптического оператора $A$ порядка $m$ в $n$-мерной области $\Omega, m \geqslant n$, оператором вида

$$
B u=g(x) \int_{M} h(\xi) u(\xi) d \xi
$$

где $M$ - гладкое подмногообразие в $\Omega$ строго меньшей размерности, $h(\xi)-$ гладкая функция на $M, g(x) \in \mathscr{D}\left(A^{2}\right)$, и из $(31)$ получаем формулу

$$
\sum_{n=1}^{\infty}\left(\mu_{n}-\lambda_{n}\right)=\int_{M} h(\xi) g(\xi) d \xi .
$$

Определенное развитие эти идеи получили в работе В.А. Любишкина и И. Д. Цопанова [95], в которой рассмотрено ограниченное возмущение самосопряженного дискретного оператора с $N(\lambda)=O\left(\lambda^{p}\right), 0<p<1 / 2$, и разобраны примеры, сводящиеся к этому случаю подобием.

В работе В. В. Дубровского [96] справедливость формулы следа

$$
\lim _{m \rightarrow \infty} \sum_{j=0}^{n_{m}}\left(\mu_{j}-\lambda_{j}-\left(B \varphi_{j}, \varphi_{j}\right)\right)=0
$$


была установлена для самосопряженного оператора $A$ с $N(\lambda)=O\left(\lambda^{p}\right)$, где $p \leqslant$ $1 / 2$, и ограниченного $B$, а для возмущения, принадлежащего классу ГильбертаШмидта, формула была доказана при $p \leqslant 1$. В продолжении этих исследований [97] автор для случая ограниченного возмущения и $p<1$ получил формулы следов для степени $n$ собственных чисел, регуляризовав их $l-1$ поправкой теории возмущений, где $l \geqslant n /(1-p)$. Во многом на основе этих абстрактных результатов в серии работ В.В. Дубровского и его учеников (см. [98] и там дальнейшие ссылки) были доказаны формулы следов для степени оператора Лапласа на треугольниках специального вида.

Различные уточнения этих результатов при дополнительных предположениях об асимптотическом поведении и структуре спектра (наличие остаточного члена в асимптотической формуле для собственных чисел и его порядок, существование лакун в спектре и их протяженность и т.п.) были получены в работах [99], [94], [100], [101] с использованием той же техники, выделим здесь работу [101], в которой удалось охватить некоторые классы операторов с неядерной резольвентой. Также укажем на исследования А.Г. Баскакова [102], в которых близкие результаты были получены методом подобных операторов, и на близкую по технике более позднюю работу [103].

В несколько отличающейся от [97] по технике оценок поправок теории возмущений работе [104] М. Достанич получил ряд интересных теорем. Рассматриваемые им невозмущенные операторы имеют спектр с особо регулярным поведением, и за счет этого автору удалось, не налагая дополнительных условий на возмущение, получить результаты для операторов с не слишком быстро растущей последовательностью собственных чисел. Формула следа (32) была доказана им для случая, когда

$$
c_{1} \leqslant\left(\lambda_{n+1}-\lambda_{n}\right) / n^{(p-1) / p} \leqslant c_{2}, \quad p>1,
$$

та же формула при более слабом условии

$$
\lim _{n \rightarrow \infty} \sum_{k=1}^{n}\left(\lambda_{n+1}-\lambda_{k}\right)^{-1}=0
$$

была доказана для случая $N(\lambda) \sim c \lambda^{p}, 0<p<2 / 3$. Несколько нестандартная формула второго следа

$$
\begin{gathered}
\lim _{k \rightarrow \infty} \sum_{m=1}^{n_{k}}\left(\mu_{m}^{2}-\lambda_{m}^{2}-2 \lambda_{m}\left(B \varphi_{m}, \varphi_{m}\right)-\sum_{l=1}^{\infty}\left(B \varphi_{l}, \varphi_{m}\right)\left(B \varphi_{m}, \varphi_{l}\right)\right. \\
\left.-\sum_{l=n_{k}+1}^{\infty}\left(B \varphi_{l}, \varphi_{m}\right)\left(B \varphi_{m}, \varphi_{l}\right) \frac{\lambda_{l}+\lambda_{m}}{\lambda_{l}-\lambda_{m}}\right)=0
\end{gathered}
$$

была доказана в работе для операторов с $N(\lambda) \sim c \lambda^{p}, 0<p<1 / 3$, и ограниченным $B$, а при дополнительных условиях регулярности $\lambda_{n}$ при $0<p<1 / 2$. Наконец, отметим, что в этой работе был рассмотрен и случай неограниченных возмущений при регулярности невозмущенного спектра и подчиненности возмущения степени $\beta<1 / 2$ невозмущенного оператора.

В 1999 году в нашей работе [105] была выдвинута гипотеза: формула следа (32) верна для $A$, имеющего ядерную резольвенту, и ограниченного $B$. В самой работе эта формула была доказана для случая (более общего, чем в [104]) 
регулярного поведения собственных чисел невозмущенного оператора $\lambda_{n}$ : последовательность $\lambda_{n} / n^{\delta}$ должна быть монотонно возрастающей при некотором $0<\delta \leqslant 1$. Однако там было сделано и важное продвижение в доказательстве этой гипотезы в целом: было доказано стремление второй поправки теории возмущений к нулю именно для операторов, удовлетворяющих условиям гипотезы. Вскоре в работе В.А. Садовничего, С. В. Конягина и В. Е. Подольского [106] была доказана одна фундаментальная лемма о числовых рядах, позволившая доказать стремление к нулю по некоторой системе контуров ядерной нормы резольвенты самосопряженного оператора с ядерной резольвентой, что дало вторую важнейшую составляющую доказательства приведенной гипотезы.

В нашей работе [17] был доказан ряд теорем, охватывающих как случай невозмущенного оператора с ядерной резольвентой, возмущенного относительно компактным оператором, так и малоизученный случай операторов с неядерной резольвентой. Приведем некоторые результаты этой работы.

Если оператор $B$ таков, что $\mathscr{D}(A) \subset \mathscr{D}(B)$ и существуют число $\delta \in[0,1)$ такое, что оператор $B A^{-\delta}$ продолжается до ограниченного, и некоторое число $\omega \in[0,1), \omega+\delta<1$, такое, что $A^{-(1-\delta-\omega)}$ - ядерный оператор, то существует подпоследовательность номеров $\left\{n_{m}\right\}_{m=1}^{\infty}$ такая, что при $\omega \geqslant \delta / l$

$$
\lim _{m \rightarrow \infty}\left(\sum_{j=0}^{n_{m}}\left(\mu_{j}-\lambda_{j}\right)+\frac{1}{2 \pi i} \int_{\Gamma_{m}} \sum_{k=1}^{l} \frac{(-1)^{k-1}}{k} \operatorname{Tr}\left(\left(B R_{0}(\lambda)\right)^{k}\right) d \lambda\right)=0 .
$$

В частности, при $\omega \geqslant \delta$ верна формула (32).

Если $A^{-1}$ - неядерный и существует число $\delta \in[0,1)$ такое, что оператор $B A^{\delta}$ продолжается до ограниченного и $A^{-(1+\delta)}$ - ядерный оператор, то существует подпоследовательность натурального ряда $\left\{n_{m}\right\}_{m=0}^{\infty}$ такая, что верна формула (32).

Аналогичные приведенным результаты были получены и для специального случая существования “больших" лакун в спектре оператора $A$, приведем один результат с прозрачной формулировкой: пусть у оператора $A$ существует подпоследовательность его собственных чисел $\left\{\lambda_{n_{m}}\right\}_{m=0}^{+\infty}$ такая, что $\lambda_{n_{m}+1}-\lambda_{n_{m}} \rightarrow$ $\infty$, пусть оператор $B \in \mathfrak{S}_{p}, p \in \mathbb{N}$, тогда верна формула $(33)$ с $(p-1)$-й поправкой. Это прямое обобщение известного результата для случая $p=1$, однако для ядерного возмущения никаких условий на невозмущенный оператор накладывать не требуется (по крайней мере для самосопряженного $A$ ). Другие теоремы обсуждаемой работы допускают для возмущений $B \in \mathfrak{S}_{p}$ стремление к нулю расстояний между собственными числами невозмущенного оператора (поясним на грубом примере: если $B \in \mathfrak{S}_{2}$, то для выполнения (32) достаточно выполнения условия $\left.\varlimsup_{n \rightarrow \infty} \sqrt{n}\left(\lambda_{n+1}-\lambda_{n}\right)>0\right)$. Приведем ряд примеров применения этих абстрактных результатов.

Формула (32) верна для самосопряженного эллиптического ПДО на компактном многообразии, возмущенного ПДО меньшего порядка, если только разность порядков операторов больше размерности многообразия. Рассмотрим в качестве оператора $A$ степень $\alpha>1$ оператора Лапласа $-\Delta$, действующего в $L_{2}([0, \pi] \times[0, \pi])$ с условиями Дирихле на границе квадрата, а в качестве $B$ оператор умножения на ограниченную измеримую (комплекснозначную) функцию $q(x, y)$. Формула первого следа была известна [98] для степени оператора 
$\alpha>93 / 80$, и доказательство опиралось на тонкие результаты теории чисел. Так как в этой задаче $\lambda_{n} \sim n^{\alpha}$, то формула следа верна для $\alpha>1$ с необходимым вычитанием одной поправки для потенциала $q(x, y) \in C^{\beta}$ с $\beta>1$. При этих условиях имеем (исходная формула (32) верна для ограниченного измеримого потенциала, гладкость нужна для абсолютной суммируемости коэффициентов Фурье и существования значений на границе):

$$
\begin{aligned}
& \sum_{k=1}^{\infty} \sum_{r_{k}<n^{2}+m^{2}<r_{k+1}}\left(\mu_{n m}-\left(n^{2}+m^{2}\right)^{\alpha}-\frac{1}{\pi^{2}} \int_{0}^{\pi} \int_{0}^{\pi} q(x, y) d x d y\right. \\
& \left.\quad+\frac{1}{\pi^{2}} \int_{0}^{\pi}\left(\int_{0}^{\pi} q(x, y) d y\right) \cos 2 n x d x+\frac{1}{\pi^{2}} \int_{0}^{\pi}\left(\int_{0}^{\pi} q(x, y) d x\right) \cos 2 m y d y\right) \\
& =\frac{1}{16}(q(\pi, \pi)+q(0, \pi)+q(\pi, 0)+q(0,0))+\frac{1}{4 \pi^{2}} \int_{0}^{\pi} \int_{0}^{\pi} q(x, y) d x d y \\
& \quad-\frac{1}{8 \pi}\left(\int_{0}^{\pi}(q(0, y)+q(\pi, y)) d y+\int_{0}^{\pi}(q(x, 0)+q(x, \pi)) d x\right) .
\end{aligned}
$$

Далее, возьмем в качестве оператора $A$ обыкновенный ДО, действующий в $L_{2}[0, \pi]$, определяемый выражением $-y^{(6)}$ и краевыми условиями $y(0)=$ $y^{(2)}(0)=y^{(4)}(0)=y(\pi)=y^{(2)}(\pi)=y^{(4)}(\pi)=0$, а в качестве $B$ возьмем оператор $q(x) y^{(2)}+p(x) y$ с областью определения $y(0)=y^{(2)}(0)=y(\pi)=y^{(2)}(\pi)=0$. Конечно, для обыкновенных операторов все задачи теории решены [11], но вычисления этим методом для оператора порядка выше четвертого практически необозримы. Во введенных обозначениях имеем $\delta=1 / 3, \omega<1 / 2$, и можно выбрать $\omega>\delta$. Таким образом, необходимо вычисление только величины $\left(B \varphi_{n}, \varphi_{n}\right)$. Так как $\varphi_{n}=\sqrt{2 / \pi} \sin n x$, то соответствующие вычисления несложны, и для $p(x)$ и $q^{\prime \prime}(x)$, гёльдеровых на окружности с показателем $\alpha>1 / 2$, формула следа имеет вид

$$
\begin{aligned}
& \sum_{n=1}^{\infty}\left(\mu_{n}-n^{6}+\frac{n^{2}}{\pi} \int_{0}^{\pi} q(x) d x-\frac{1}{\pi} \int_{0}^{\pi} p(x) d x-\frac{1}{4 \pi}\left(q^{\prime}(\pi)-q^{\prime}(0)\right)\right) \\
& \quad=\frac{1}{2 \pi} \int_{0}^{\pi} p(x) d x-\frac{1}{4}(p(0)+p(\pi))+\frac{1}{8 \pi}\left(q^{\prime}(\pi)-q^{\prime}(0)\right)-\frac{1}{16}\left(q^{\prime \prime}(0)+q^{\prime \prime}(\pi)\right) .
\end{aligned}
$$

Рассмотрим в качестве $A$ степень $2+\varepsilon$ оператора $-\Delta, \varepsilon>0$, действующего в $L_{2}([0, \pi] \times[0, \pi])$ с условиями Дирихле на границе квадрата, а в качестве $B$ ДО первого порядка $q \frac{\partial}{\partial x}+p \frac{\partial}{\partial y}$, где периодические продолжения функций $p_{y}^{\prime}(x, y)$, $q_{x}^{\prime}(x, y)$ имеют гладкость $C^{\beta}, \beta>1$. Так как $\lambda_{n} \sim n^{2+\varepsilon}$, то можно взять $\delta=1 /(4+2 \varepsilon)$ и $\delta+\omega=1 / 2$ и, следовательно, можно принять $\omega>\delta$. Подчеркнем, что в этой работе впервые была получена формула следа для возмущения оператора в частных производных оператором в частных производных.

Применение леммы работы [106] в работе [107] позволило получить новый результат в теории возмущений унитарных операторов: для пары унитарных операторов $U_{j}, j=1,2$, с собственными числами $\lambda_{j n}$ такими, что $\sum_{n=-\infty}^{+\infty}\left|\lambda_{j n}-1\right|<+\infty$, доказана формула первого следа и вычислен ответ.

В завершение обсуждения результатов для абстрактных операторов отметим работы Х. Х. Муртазина и З. Ю. Фазуллина [108], [109], в которых доказано, что 
формула (32) для самосопряженных операторов (техника работ существенно использует самосопряженность) верна и при более слабом требовании, нежели ядерность резольвенты невозмущенного оператора, а именно при условии $\lambda_{n}^{-1}=o\left(n^{-1}\right)$, а также получены новые результаты и для компактных возмущений операторов с неядерной резольвентой.

Как мы уже отмечали во введении, в последние два десятилетия было решено много отдельных важных задач с применением (в той или иной степени) индивидуально разработанных подходов.

Формула следа для оператора Штурма-Лиувилля (9) долгое время считалась доказанной Л. А. Диким для максимального класса потенциалов. Однако недавно появился ряд работ с усилением этого результата. Предварила их статья В.В. Дубровского [28], в которой были найдены условия абсолютной сходимости ряда разностей собственных чисел. Он представил потенциал в виде суммы двух функций $q(x)=q_{1}(x)+q_{2}(x), q_{1}(x)=\sum_{n=1}^{\infty} a_{2 n} \cos 2 n x, a_{i}-$ коэффициенты ряда Фурье по косинусам функции $q(x)$, и доказал, что если $q_{1}(x)$ - непрерывная функция с абсолютно сходящимся рядом Фурье, то ряд разностей собственных чисел сходится абсолютно и $2 \sum_{n=1}^{\infty}\left(\mu_{n}-n^{2}\right)=q_{1}(0)$.

В 1999 году В.А. Винокуров и В.А. Садовничий [29] доказали, что собственные числа оператора Штурма-Лиувилля являются дифференцируемыми функционалами аргумента $q(x) \in L_{1}[0, \pi]$ и доказали формулу (8) для суммируемых потенциалов, причем для потенциалов, локальные характеристики которых в окрестностях концов отрезка удовлетворяют условиям сходимости ряда Фурье, по-прежнему верна формула (9).

А. М. Савчук [31] и независимо В.А. Винокуров и В.А. Садовничий [30] рассмотрели оператор Штурма-Лиувилля с "потенциалом" $\delta$-функцией. В работе [31] такой оператор аккуратно определен с помощью квазипроизводных (см. [46]):

$$
-\left(y^{\prime}-u(x) y\right)^{\prime}-u(x)\left(y^{\prime}-u(x) y\right)-u^{2}(x) y,
$$

здесь $u(x)=1$ при $x \geqslant \pi / 2, u(x)=0$ при $x<\pi / 2$, и условия склейки $y^{\prime}\left(\frac{\pi}{2}+0\right)-y^{\prime}\left(\frac{\pi}{2}-0\right)=y\left(\frac{\pi}{2}\right)$, а в [30] аналогичная задача с потенциалом $\sum_{i} c_{i} \delta\left(x-x_{i}\right)$ сведена к краевой задаче для интегрального уравнения

$$
z(x)=x-\int_{0}^{\pi} \nu(x-t) z(t) d(\sigma(t)+\lambda t), \quad z(\pi)=0,
$$

здесь $\sigma(t)$ - функция ограниченной вариации на отрезке, непрерывная справа и непрерывная в граничных точках (фактически $\sigma(t)$ - обобщенная первообразная потенциала и совпадает с $u(t)$ из дифференциального выражения в квазипроизводных работы [31]). В [30] получена общая формула следа

$$
\sum_{n=1}^{\infty}\left(\mu_{n}-n^{2}-\frac{2}{\pi} \int_{0}^{\pi} \sin ^{2} n x d \sigma(x)\right)=-\frac{1}{8} \sum_{i} c_{i}^{2},
$$

где $c_{i}$ - величины скачков функции $\sigma(x)$, сумма берется по всем скачкам. Отметим, что в класс потенциалов, охваченных этой работой, попадают функции, интегрируемые по Данжуа-Перрону в узком смысле.

В работе А. М. Савчука и А. А. Шкаликова [32] независимо получены те же результаты, что и в [30], и сверх того при дополнительных условиях гладкости 
в граничных точках, наложенных уже на обобщенную первообразную потенциала, доказана суммируемость по Чезаро формулы следа вида (9):

$$
(C, 1) \sum_{n=1}^{\infty}\left(\mu_{n}-n^{2}\right)=-\frac{u^{\prime}(0)+u^{\prime}(\pi)}{4}-\frac{1}{8} \sum_{i} c_{i}^{2},
$$

при условии дифференцируемости в 0 и $\pi$ функции $u(x)=\int q(x) d x$.

Ряд интересных результатов был получен и в задачах на неограниченных интервалах. Т. Г. Амангильдин в нескольких работах (см. [110] для дальнейших ссылок) для оператора в $L_{2}[0,+\infty)$

$$
-y^{\prime \prime}+q(x) y=\lambda y, \quad y(0)=0,
$$

при выполнении следующих условий на потенциал на множестве $x \geqslant x_{0}$ : $q(x)>0, q^{-1 / 4}(x)$ - выпукла, $q^{(3)} \geqslant \varepsilon>0, q^{(4)} \geqslant 0,\left|\left(\ln ^{-1} q^{(3)}(x)\right)^{\prime}\right|<C$, интегралы

$$
\int_{x_{0}}^{\infty} q^{-1 / 2}(x) d x \quad \text { и } \quad \int_{x_{0}}^{\infty}\left(q^{2}(x) q^{-5 / 2}(x)+q^{\prime \prime}(x) q^{-3 / 2}(x)\right) d x
$$

сходятся, доказал, что если возмутить оператор оператором умножения на финитную функцию с нулевым средним, то верна формула $\sum_{n}\left(\mu_{n}-\lambda_{n}\right)=-(\widetilde{q}(0)-$ $q(0)) / 4$. Он же рассмотрел задачу (34) в специальном случае $q(x)=x^{\alpha}, \alpha>0$, и для финитного возмущения явно вычислил сумму $\sum\left(\mu_{n}-\lambda_{n}-a n^{-2 /(2+\alpha)}\right)$.

Г. В. Козлов и В. А. Любишкин [111] рассмотрели оператор

$$
(-1)^{n} y^{(2 n)}+\left(x^{\alpha}+q(x)\right) y=\lambda y, \quad y(0)=y^{\prime}(0)=\cdots=y^{(n-1)}(0)=0,
$$

в $L_{2}[0,+\infty)$, где $q(x) \in C^{2}-$ финитная функция, а $\alpha>0$. С применением суммирования по Абелю при $\frac{2 n \alpha}{2 n+\alpha}>1$ была доказана формула следа

$$
\sum_{k}\left(\mu_{k}-\lambda_{k}-B k^{-\frac{2 n}{2 n+\alpha}} \int_{0}^{+\infty} q(x) d x\right)=\frac{q(0)}{4}-B \zeta\left(\frac{2 n}{2 n+\alpha}\right) \int_{0}^{+\infty} q(x) d x,
$$

где

$$
B=\frac{\alpha}{2 n+\alpha}\left(\frac{\pi}{\alpha} B\left(\frac{1}{\alpha}, 1+\frac{1}{2 n}\right)\right)^{-\frac{\alpha}{2 n+\alpha}} .
$$

Отдельно в работе доказано, что эта формула верна и для гармонического осциллятора $n=1, \alpha=2$. Те же авторы в [112] получили для гармонического осциллятора при финитном возмущении $q(x) \in C^{4}$ второй след. Мы вернемся к обсуждению некоторых результатов о гармоническом осцилляторе ниже, в контексте обсуждения метода суммирования по Абелю регуляризованных следов.

Задачу для ДО высокого порядка на полуоси, заданного дифференциальным выражением

$$
(-1)^{m} y^{(2 m)}+p_{2 m-2}(x) y^{(2 m-2)}+\cdots+p_{0}(x) y
$$

и граничными условиями $y(0)=y^{\prime \prime}(0)=\cdots=y^{(2 m-2)}(0)=0$, где $p_{2 i}(x)-$ действительные локально интегрируемые функции, рассмотрели недавно 
А. И. Козко и А. С. Печенцов [113]. Предполагая о рассматриваемом операторе его дискретность и полуограниченность, они для возмущения оператором умножения на финитную действительнозначную измеримую ограниченную функцию $q(x)$ доказали, что если функция

$$
\psi(x)=\frac{1}{x} \int_{0}^{x} q(t) d t
$$

имеет ограниченную вариацию в некоторой правосторонней окрестности нуля, то верна формула следа

$$
\begin{aligned}
& \sum_{k=1}^{\infty}\left(\mu_{k}-\lambda_{k}-\frac{c_{k}}{\pi} \int_{0}^{+\infty} q(t) d t\right)=-\frac{\psi(+0)}{4}, \\
& c_{1}=\lambda_{1}^{\frac{1}{2 m}}, \quad c_{k}=\lambda_{k}^{\frac{1}{2 m}}-\lambda_{k-1}^{\frac{1}{2 m}}, \quad k=2,3, \ldots
\end{aligned}
$$

Отметим, что авторы использовали обсуждавшуюся выше лемму Гасымова [10].

Неполуограниченный случай для оператора (34) исследовал В. А. Любишкин (см. [114]). Он рассмотрел неограниченно убывающий потенциал $q(x) \in$ $C^{3}[0,+\infty)$ такой, что $q(x)<0, q^{\prime}(x)<0, q^{\prime}(x)=O\left(|q(x)|^{3 / 2-\varepsilon}\right)$, интегралы

$$
\int_{0}^{+\infty}|q(x)|^{\varepsilon-1 / 2} d x \quad \text { и } \quad \int_{0}^{+\infty}\left(q^{\prime 2}(x)-q(x)\left|q^{\prime \prime}(x)\right|\right)|q(x)|^{\varepsilon-5 / 2} d x
$$

сходятся, и возмущением был финитный потенциал. Тогда для заданного $k$ были найдены достаточные условия существования и равенства нулю $k$-го регуляризованного следа.

Другой тип сингулярности оператора изучен в [115]: в $L_{2}\left(-1,1 ;(1-x)^{\alpha}(1+\right.$ $\left.x)^{\beta}\right), \alpha, \beta>-1$, рассмотрено возмущение оператора типа Якоби

$$
T y=-\left(1-x^{2}\right) y^{\prime \prime}-(\beta-\alpha-(\alpha+\beta+2) x) y^{\prime}
$$

оператором умножения на абсолютно непрерывную функцию $p(x)$ и получены формулы следов, например, при $\alpha=\beta=\frac{1}{2}$

$$
\sum_{j=0}^{+\infty}\left(\mu_{j}-\lambda_{j}-\frac{1}{\pi} \int_{0}^{\pi} p(\cos \theta) d \theta\right)=\frac{1}{2 \pi} \int_{0}^{\pi} p(\cos \theta) d \theta-\frac{p(1)+p(-1)}{4} .
$$

Аналогично исследован в [100] и оператор типа Лежандра.

В работе В.Е. Подольского [116] в рамках решения обратной задачи для оператора Штурама-Лиувилля по взвешенной $\zeta$-функции для задачи на полуоси при естественных ограничениях на потенциалы $q_{i}(x)$ доказаны формулы следов всех порядков, например,

$$
\begin{gathered}
\int_{-\infty}^{+\infty} d\left(\rho_{1}(\lambda)-\rho_{2}(\lambda)\right)=h_{2}-h_{1}, \\
\int_{-\infty}^{+\infty} \lambda d\left(\rho_{1}(\lambda)-\rho_{2}(\lambda)\right)=h_{1}^{3}-h_{2}^{3}+\frac{1}{4}\left(q_{1}^{\prime}(0)-q_{2}^{\prime}(0)\right)-h_{1} q_{1}(0)+h_{2} q_{2}(0) .
\end{gathered}
$$


Отметим конструкцию $\zeta$-функции как суммы трех функций с изначально непересекающимися областями определения:

$$
Z_{1}(s)=\int_{-\infty}^{-\varepsilon} \lambda^{-s} d \rho(\lambda), \quad Z_{2}(s)=\int_{-\varepsilon}^{+\varepsilon} \lambda^{-s} d \rho(\lambda), \quad Z_{3}(s)=\int_{+\varepsilon}^{+\infty} \lambda^{-s} d \rho(\lambda),
$$

здесь $Z_{1}(s)$ определена во всей плоскости, $Z_{2}(s)$ определена при $\operatorname{Re} s<0$ и $s=0, Z_{3}(s)$ определена при $\operatorname{Re} s>1 / 2$. В сумме $Z(s)=Z_{1}(s)+Z_{2}(s)+Z_{3}(s)$ участвует аналитическое продолжение $Z_{3}(s)$ в полуплоскость $\operatorname{Re} s>-\beta$, существующее при $q(x) \in C^{\beta}[0, \delta]$. Можно в очередной раз отметить, что в самых разнообразных методах спектральной теории ДО второго порядка всегда важную роль играет гладкость потенциала в некоторой окрестности нуля.

Замечательное применение метода функций класса $K$ нашла Е. В. Александрова, рассмотрев [117] задачу о малых поперечных колебаниях трубы, находящейся в вязкой среде и несущей установившийся поток несжимаемой жидкости

$$
\begin{gathered}
y^{(4)}(1+\alpha \lambda)+v^{2} y^{\prime \prime}+2 \beta v \lambda y^{\prime}+\lambda(\lambda+k) y=0, \\
y(0)=y(1)=y^{\prime \prime}(0)=y^{\prime \prime}(1)=0,
\end{gathered}
$$

здесь $\lambda$ - спектральный параметр, прочие числовые параметры имеют физический смысл. В этой задаче со сложным вхождением спектрального параметра характеристический определитель является аналитической функцией с существенной особенностью и спектр имеет две точки накопления. Автор столкнулся с невозможностью разделить соответствующие серии собственных чисел, и в формуле следа появился дефект нумерации $\varkappa$ :

$$
\sum_{n=1}^{\infty}\left(\lambda_{n}+\alpha \pi^{4} n^{4}+\frac{k \alpha-1}{\alpha}\right)+\sum_{n=1}^{\infty}\left(\lambda_{n, \alpha}+\frac{1}{\alpha}\right)=\frac{\beta^{2} v^{2}}{4 \alpha}-\frac{\varkappa}{\alpha} .
$$

Неизвестно, можно ли в этой задаче занумеровать собственные числа так, чтобы в обеих сериях нумерация совпадала с асимптотической, и хотя автор нашел выражение для дефекта через $\zeta$-функцию задачи, однако сложность конструкции $\zeta$-функции не дает возможности явно вычислить этот дефект.

Метод суммирования по Абелю регуляризованных следов, восходящий к методу В.Б. Лидского [118] суммирования разложений по Абелю, впервые использованный в конкретных задачах В. А. Любишкиным в работах [99], [112], был в более общем виде исследован в статье В. А. Любишкина и В. Е. Подольского [23]. Известно, что для дифференциального полуограниченного самосопряженного эллиптического оператора $A$ порядка $m$ на компактном многообразии размерности $n$ и оператора $B$ умножения на гладкую функцию существует асимптотическое разложение при $t \rightarrow 0+$ следа полугрупп $\exp (-t A)$ и $\exp (-t(A+B))$ по степеням $t^{(j-n) / m}, j=0,1,2, \ldots$. В работе при условии $m>n$ было доказано существование и дан полный алгоритм вычисления набора коэффициентов $\left\{\varkappa_{l}\right\}$ таких, что верна формула

$$
\lim _{t \rightarrow 0+} \sum_{k=1}^{\infty}\left(\mu_{k}-\lambda_{k}+\sum_{l=0}^{n} \varkappa_{l} \lambda_{k}^{-\frac{l}{m}}\right) e^{-\lambda_{k} t}=\varkappa_{n+1} .
$$


Сильный метод сумирования (что само по себе, конечно, недостаток, и всякая возможность избежать его должна тщательно исследоваться) позволяет получать простую регуляризацию канонической структуры - конечным набором степеней собственных чисел невозмущенного оператора. В работе [20] В. Е. Подольский предложил для суммы $\sum_{l=0}^{n} \varkappa_{l} \lambda_{k}^{-\frac{l}{m}}$ термин слабая асимптотика (в смысле суммирования по Абелю) последовательности $\mu_{n}$, а в работе [119] он же распространил эту технику на степенно-логарифмические асимптотики.

Суммирование по Абелю регуляризованных следов было применено в работе Б. Саймона с сотрудниками [24], мы вернемся к ней в дополнении, посвященном формуле Крейна. Крайне интересным представляется изучение связи теории регуляризованных следов и различных методов суммирования с теориями регуляризаций и перенормировок в различных разделах современной физики, например, в работе [120] рассматриваются весьма интересные вариации на тему суммирования посредством $\zeta$-функции и $\theta$-функции в задаче о вычислении энергии Казимира. Таким задачам ${ }^{10}$ посвящено в последние годы немало работ, цитирование которых выходит за рамки нашего обзора, укажем лишь на обзор [121], содержащий описание нескольких приложений теории к физическим и математическим задачам.

Суммирование по Абелю было применено в [122] к одномерному гармоническому осциллятору и его ограничению на полуось: при возмущении оператором умножения на функцию $p(x)=q(x) / x^{\alpha}, 0 \leqslant \alpha<1$, где $|q(x)-q(0)|<C_{1} x^{\delta_{1}}$ при $x \leqslant A, \delta_{1}>\alpha$ и $|q(x)| \leqslant C_{2} / x^{\delta_{2}}$ при $x>A, \delta_{2}>2-\alpha$, была доказана справедливость формулы следа

$$
\begin{gathered}
\lim _{t \rightarrow 0+} \sum_{n=0}^{\infty} e^{-\lambda_{n} t}\left(\mu_{n}-\lambda_{n}+\frac{q(0) \Gamma\left(\frac{1-\alpha}{2}\right)}{\sqrt{\pi} \Gamma\left(\frac{\alpha}{2}\right)} \lambda_{n}^{-1+\frac{\alpha}{2}}-\frac{2 \lambda_{n}^{-\frac{1}{2}}}{\pi} \int_{0}^{\infty} q(t) t^{-\alpha} d t\right) \\
\quad=\frac{4 \Gamma\left(\frac{1-\alpha}{2}\right) Z\left(1-\frac{\alpha}{2}\right) \sin \frac{\pi \alpha}{2}}{\pi \sqrt{\pi}(2-\alpha)} q(0)-\frac{4}{\pi \sqrt{\pi}} Z\left(\frac{1}{2}\right) \int_{0}^{+\infty} q(t) t^{-\alpha} d t,
\end{gathered}
$$

где $Z(s)=\sum_{k=0}^{\infty}(4 k+3)^{-s}$. Позже Е. В. Александрова [123], действуя аналогично методу работы [19], для более узкого класса потенциалов сняла суммирование в этой формуле.

Другим методом, более близким классическому использованию $\zeta$-функции, основываясь на точных асимптотиках для собственных чисел, недавно формулы следов всех порядков для одномерного гармонического осциллятора получили А. Пушницкий и Я. Соррель [124], разумеется, для гладкого потенциала. Однако отметим, что получение асимптотик базируется на разложении для $\theta$-функции, полученном в работе М. Хитрика и И. Полтеровича [125].

Завершая обсуждение современного состояния метода суммирования по Абелю регуляризованных следов, отметим работу В.Е. Подольского [126], в которой изучение и применения метода были перенесены с ДО и ПДО на абстрактные операторы. В качестве невозмущенного оператора рассматривается генератор аналитической полугруппы, вообще говоря, несамосопряженный, и

\footnotetext{
${ }^{10}$ Например, просто в контексте основных формул регуляризованных следов типа (5) и (6) интересна формальная с точки зрения математика цепочка равенств (так как интерес эта цепочка вызывает в случае неограниченности оператора $A) \sum\left(\varphi_{i}, A \varphi_{i}\right)=\operatorname{Tr} A=\sum \lambda_{i}=$ $\left.\sum \lambda_{i}^{-s}\right|_{s=-1}=\zeta_{A}(-1)$ для квантовой энергии вакуума.
} 
в работе получен ряд оценок ядерной нормы полугрупп, даны условия сходимости ряда теории возмущений полугрупп

$$
e^{-t(A+B)}=e^{-t A}+\sum_{k=1}^{+\infty}(-1)^{k} \int_{0}^{t} \int_{0}^{t_{1}} \cdots \int_{0}^{t_{k-1}} e^{-\left(t-t_{1}\right) A} B \cdots B e^{-t_{k} A} d t_{k} \cdots d t_{1}
$$

в ядерной норме на отрезках, включающих точку 0. Эти результаты применены к доказательству формул следов и асимптотик возмущенной полугруппы в нуле. Доказано выполнение формулы

$$
\lim _{t \rightarrow 0+} \sum_{n=1}^{\infty}\left(\mu_{n}-\lambda_{n}-\left(B \varphi_{n}, \varphi_{n}\right)\right) e^{-\lambda_{n} t}=0
$$

при выполнении условия $\left|\lambda_{n}\right|^{-1}=o\left(n^{-1}\right)$ и ограниченном возмущении (cp. с peзультатами работы [109], в которой аналогичная формула получена без суммирования по Абелю, но для самосопряженного случая). Также в работе найдены достаточные условия на пару операторов, позволяющие в общем виде получать асимптотику следа возмущенной полугруппы

$$
\operatorname{Tr} e^{-t(A+B)}=\operatorname{Tr} e^{-t A}-t \operatorname{Tr}\left(B e^{-t A}\right)+\frac{t^{2}}{2} \operatorname{Tr}\left(B^{2} e^{-t A}\right)+O\left(t^{\omega}\right), \quad t \rightarrow+0,
$$

с эффективно найденным остаточным членом и, вообще говоря, неограниченным возмущающим оператором $B$; например, для двумерного оператора Лапласа с комплексным (не обязательно бесконечно гладким) потенциалом $\omega=3 / 2$.

Еще в середине 60-х годов И. М. Гельфанд указывал на оператор ЛапласаБельтрами на двумерной сфере как на следующий принципиальный рубеж для теории следов дискретных операторов. Оператор Лапласа-Бельтрами на компактных симметрических пространствах ранга 1 (это сферы и проективные пространства) естественно возникает при делении переменных у многомерного гармонического осциллятора; мы рекомендуем читателю за дальнейшими подробностями (связанными со спектральной геометрией, статистической физикой, следами и т.п.) обратиться к монографии Н. Харта [127]. Предваряя обсуждение конкретных результатов, укажем на то, что оператор Лапласа на сфере является стандартным примером, подтверждающим неулучшаемость оценки Л. Хёрмандера остаточного члена асимптотики $N(\lambda)=a \lambda^{n / m}+O\left(\lambda^{(n-1) / m}\right)$ для эллиптического ДО, здесь $m$ - порядок оператора, $n$ - размерность многообразия. Причина этого в неограниченно возрастающей кратности "редко" расположенных собственных чисел, однако именно это позволяет изучить распределение собственных чисел в группе, образующейся после возмущения из кратного собственного числа. Получающиеся формулы следа могут в некоторых задачах служить частичной компенсацией неуточняемости оценки остатка в формуле для $N(\lambda)$.

Первые результаты по этой задаче получил в 1983 году В. В. Дубровский [128] для возмущения оператора Лапласа-Бельтрами на двумерной сфере потенциалом некоторого специального класса, затем в 1991 году В. А. Садовничий и 
В. В. Дубровский (полное доказательство и ссылки см. в [18]) доказали формулу со скобками первого регуляризованного следа для оператора ЛапласаБельтрами на двумерной сфере, возмущенного гладким нечетным комплекснозначным потенциалом $q(x)$. В 1994 году В. Е. Подольский [19], применив к этой задаче суммирование по Абелю и затем к полученной формуле тауберову теорему Литлвуда, доказал, что ряд сходится без скобок, далее А.Н. Бобров и В. Е. Подольский [129], точно исследовав абсолютную и условную сходимости регуляризованных следов степеней оператора Лапласа, в частности, доказали, что полученный в [19] ряд

$$
\sum_{n=0}^{\infty}\left(\mu_{n}-\lambda_{n}\right)=-\frac{1}{8 \pi} \int_{S^{2}} q^{2}(x) d S
$$

сходится абсолютно.

Перейти от нечетного потенциала к произвольному и на сферы любой размерности удалось В. Е. Подольскому [20], он доказал формулы следов со скобками всех порядков, а также просуммированные по Абелю для любого гладкого потенциала и на сферах любой размерности. В этой работе впервые были получены следы операторов в частных производных в случае, когда порядок оператора меньше размерности многообразия.

А.Н. Бобров [130] рассмотрел задачу в общей постановке: для пары (оператор, многообразие) такой, что все траектории гамильтонова потока, индуцированного оператором на многообразии, замкнуты, он получил формулы следов всех порядков со скобками и просуммированные по Абелю. Приведем одну из явно вычисленных формул. На поверхности Цолля, определяемой заданием на сфере $S^{2}$ метрики $g=(1+h(\cos \theta))^{2} d \theta^{2}+\sin ^{2} \theta d \varphi^{2}$, где $C^{\infty} \ni h:[-1,1] \rightarrow(-1,1)-$ некоторая нечетная функция, $h(1)=0$, для собственных чисел оператора Лапласа-Бельтрами, возмущенного гладким потенциалом $q(\theta, \varphi)$, верно

$$
\begin{gathered}
\sum_{k=0}^{\infty} \sum_{i=0}^{2 k}\left(\lambda_{k i}-k(k+1)-\frac{1}{4 \pi} \int_{0}^{2 \pi} \int_{0}^{\pi} q(\theta, \varphi)(1+h(\cos \theta)) \sin \theta d \theta d \varphi\right) \\
=-\frac{1}{24 \pi} \int_{0}^{2 \pi} \int_{0}^{\pi}\left(\Delta q(\theta, \varphi)-2 q(\theta, \varphi)(\gamma(\theta)+1)+3 q^{2}(\theta, \varphi)\right) \\
\quad \times(1+h(\cos \theta)) \sin \theta d \theta d \varphi \\
+\frac{1}{30} \int_{0}^{\pi}\left(1-\Delta \gamma(\theta)-\gamma^{2}(\theta)\right)(1+h(\cos \theta)) \sin \theta d \theta
\end{gathered}
$$

здесь $\gamma(\theta)=\left(1+h(\cos \theta)-\cos \theta h^{\prime}(\cos \theta)\right) /(1+h(\cos \theta))^{3}$ - гауссова кривизна рассматриваемой поверхности. В этой формуле впервые был явно просчитан случай произвольного, а не только нечетного потенциала на сфере (каноническая сфера соответствует $h \equiv 0)$, и, что особенно примечательно, в случае, когда $q(\theta, \varphi) \equiv 0$, формула становится чисто геометрической. Также на этот общий случай были распространены результаты [129], а именно для рядов с единой 
(по возрастанию) нумерацией собственных чисел вида

$$
\sum_{n=0}^{\infty}\left(\mu_{n}^{\alpha}-\lambda_{n}^{\alpha}-\sum_{j=0}^{2[\alpha]+n-1} \chi_{j}(\alpha) \lambda_{k}^{-(j-2 \alpha) / 2}\right)
$$

для которых доказана суммируемость по Абелю, точно исследовано, при каких $\alpha$ этот ряд сходится абсолютно, при каких условно и при каких расходится.

Значительные трудности приносит в эту задачу отказ от бесконечно дифференцируемых потенциалов, так как тогда нельзя применять технику теории ПДО. Прямым исследованием поправок теории возмущений В. А. Садовничему и З. Ю. Фазуллину [131] удалось получить формулы следов для оператора Лапласа-Бельтрами на двумерной сфере при условии принадлежности потенциала классу дважды непрерывно дифференцируемых функций.

Многомерные дискретные операторы, действующие в $L_{2}$ на всем пространстве, являются одной из наиболее трудных задач современной теории. В работе В. А. Любишкина [132] рассматривалось возмущение оператора двумерного гармонического осциллятора $-\frac{\partial^{2}}{\partial x_{1}^{2}}-\frac{\partial^{2}}{\partial x_{2}^{2}}+x_{1}^{2}+x_{2}^{2}$ оператором умножения на гладкую финитную зависящую только от радиуса функцию, обращающуюся в нуль в окрестности нуля. Из-за симметричности возмущения этот случай мало отличается от одномерного, но даже это явилось заметным продвижением вперед. З. Ю. Фазуллин и Х. Х. Муртазин [133] рассмотрели возмущение того же оператора оператором умножения на финитную функцию $q\left(x_{1}, x_{2}\right) \in C_{0}^{4}\left(\mathbb{R}^{2}\right)$ и доказали следующую формулу первого следа:

$$
\begin{aligned}
& \sum_{n=0}^{\infty}\left(\sum_{k=0}^{n}\left(\mu_{k n}-\lambda_{k n}\right)-\frac{1}{2 \pi} \iint_{\mathbb{R}^{2}} q\left(x_{1}, x_{2}\right) d x_{1} d x_{2}\right) \\
& \quad=\frac{1}{4 \pi} \iint_{\mathbb{R}^{2}}\left(q\left(x_{1}, x_{2}\right)-\frac{1}{2} q^{2}\left(x_{1}, x_{2}\right)-\left(x_{1}^{2}+x_{2}^{2}\right) q\left(x_{1}, x_{2}\right)\right) d x_{1} d x_{2} .
\end{aligned}
$$

Х. Х. Муртазин и З. Ю. Фазуллин исследовали еще одну интереснейшую задачу математической физики [134] - они изучили спектр и получили формулу следа для двумерного оператора Шрёдингера в однородном магнитном поле:

$$
\left(-i \frac{\partial}{\partial x_{1}}-x_{2}\right)^{2}+\left(-i \frac{\partial}{\partial x_{2}}-x_{1}\right)^{2}+V\left(x_{1}, x_{2}\right)
$$

здесь $V$ - электрический потенциал. При $V \equiv 0$ спектр оператора состоит из чисел $\lambda_{n}=2(2 n+1), n=0,1,2, \ldots$, причем каждое бесконечнократно. В работе рассмотрено возмущение $V$ - вещественная функция из $L_{2}^{\text {loc }}\left(\mathbb{R}^{2}\right)$ такая, что оператор умножения на $V$ является относительно компактным возмущением (например, достаточно $V \rightarrow 0$ при $|x| \rightarrow \infty$ ). Тогда спектр возмущенного оператора можно разбить на серии $\left\{\mu_{n}^{m}\right\}_{m=-n}^{\infty}, n=0,1,2 \ldots$, так, что при финитной $V$ имеет место сходимость ряда

$$
\sum_{n=0}^{\infty}\left(\sum_{m=-n}^{\infty}\left(\lambda_{n}-\mu_{n}^{m}\right)+\frac{1}{\pi} \int_{\mathbb{R}^{2}} V\left(x_{1}, x_{2}\right) d x_{1} d x_{2}\right)=\frac{1}{8 \pi} \int_{\mathbb{R}^{2}} V^{2}\left(x_{1}, x_{2}\right) d x_{1} d x_{2} .
$$


Несколько позже независимо аналогичный результат получили Е. Коротаев и А. Пушницкий [135].

Едва ли не единственные примеры явно вычисленных формул следа для компактного оператора даны в статье М. Достанича [136]. Для ИО

$$
A f(x)=\int_{-1}^{1}|x-y|^{\alpha-1} f(y) d y, \quad 0<\alpha<1,
$$

формула следа

$$
\sum_{n=1}^{\infty}\left(\lambda_{n}-\frac{2^{\alpha+1} \Gamma(\alpha) \cos \frac{\alpha \pi}{2}}{\pi^{\alpha} n^{\alpha}}\right)=-4^{\alpha} \zeta(1-\alpha)
$$

получена автором с помощью представления $A$ в виде суммы $B+R$, где $B$ есть ИО с ядром

$$
\sum_{n=-\infty}^{+\infty}\left(|x-y+4 n|^{\alpha-1}-|x+y+4 n+2|^{\alpha-1}\right)
$$

a $R$ имеет сингулярные числа, убывающие быстрее любой степени. Для оператора

$$
L f(x)=-\frac{1}{\pi} \int_{-1}^{1} \ln |x-y| f(y) d y
$$

в этой работе предъявлена формула

$$
\frac{\pi}{2} \sum_{n=1}^{\infty}\left(\lambda_{n}-\frac{2}{\sqrt{4+\pi^{2} n^{2}}}\right)=-\gamma-\ln 2-\sqrt{2} \int_{0}^{\infty}\left(t+\frac{t^{2}}{2}\right)^{-\frac{1}{2}}\left(e^{4(1+t)}-1\right)^{-1} d t
$$

где $\gamma$ - постоянная Эйлера.

Техника ПДО впервые была использована в теории следов Ю. В. Коганом [137], В. Е. Подольским [138] и затем А. Н. Бобровым и В. Е. Подольским в работах об операторе Лапласа-Бельтрами на многообразиях с периодическим геодезическим потоком. Характерным примером применения этой техники можно считать работу А.Н. Боброва [139], где исследованы граничные задачи для операторов в частных производных со спектральным параметром в граничных операторах и в качестве примера рассмотрена классическая задача Стеклова об установившейся температуре

$$
\Delta u(x)=0 \quad \text { при } \quad x \in \Omega, u_{n}^{\prime}(x)=\lambda g(x) u(x) \quad \text { при } \quad x \in \partial \Omega,
$$

где $\Omega$ - ограниченная односвязная область на плоскости с границей $\partial \Omega \in C^{\infty}$, $n$ - внешняя нормаль к $\partial \Omega$, а $g(x)>0, g(x) \in C^{\infty}(\partial \Omega)$ (в [137] эта задача рассматривалась при $g(x) \equiv 1)$. Формула следа в этой задаче имеет вид

$$
\begin{aligned}
\lambda_{-1} & +\sum_{n=0}^{\infty}\left(\lambda_{2 n}+\lambda_{2 n+1}-4 \pi n\left(\int_{0}^{2 \pi} a(x) d x\right)^{-1}\right) \\
& =\frac{1}{12 \pi} \int_{0}^{2 \pi} \frac{\left(b^{\prime}(x) a(x)-a^{\prime}(x) b(x)\right)^{2}-a^{2}(x) b^{2}(x)}{b(x) a^{3}(x)} d x+\frac{\pi}{3 \int_{0}^{2 \pi} a(x) d x},
\end{aligned}
$$

где $a(x)=g\left(F\left(e^{i x}\right)\right), b(x)=\left|W^{\prime}\left(F\left(e^{i x}\right)\right)\right|, W$ - конформное отображение области $\Omega$ на единичный круг, $F$ - обратное к $W$ отображение. 


\section{Дополнение 1. Функция спектрального сдвига и формула Крейна}

В 1947 году в работах [140], [141] И. М. Лифшиц рассмотрел возмущение спектра дискретных операторов при конечномерном возмущении и нашел некоторые формулы и количественные соотношения для величины сдвига собственных чисел, причем во второй работе им было рассмотрено семейство операторов, зависящее от малого параметра $\alpha$ так, что предельный оператор имеет непрерывный спектр, и полученные соотношения для возмущенного спектра были подвергнуты еще и асимптотическому анализу по $\alpha$. В этих работах впервые появилась функция спектрального сдвига ${ }^{11} \xi(\lambda)$ и были получены формулы для нее в случае конечномерного возмущения. Далее И. М. Лифшиц продолжил эти исследования и в статье [3] применил их к задаче о вычислении следа оператора $F(L+\Lambda)-F(L)$, где $L$ - невозмущенный эрмитов оператор, $\Lambda$ - конечномерный оператор возмущения, а $F(x)$ - некоторая (принадлежащая достаточно широкому классу) функция, и получил (с применением не вполне корректных рассуждений) формулу (7). Заслуживает особого внимания физический пример, рассмотренный в работе: если за $L$ обозначить оператор, описывающий колебания кристаллической решетки, то свободная энергия колебаний записывается в виде $F=\operatorname{Tr} \Phi(L)$, где

$$
\Phi(\lambda)=\theta \ln (1-\exp (-\hbar \sqrt{\lambda} / \theta))+\frac{1}{2} \hbar \sqrt{\lambda},
$$

$\theta$ - температура, $\hbar$ - постоянная Планка, и полученная формула следа позволила вычислить изменение свободной энергии колебаний кристаллической решетки при внесении чужеродной примеси в кристалл.

Вскоре после работы И. М. Лифшица [3] в 1953 году вышла знаменитая работа М. Г. Крейна [4], в которой формула следа работы [3] получила строгое обоснование. М.Г. Крейн доказал, что для самосопряженного оператора $H$ и самосопряженного ядерного возмущения $T$ верна формула

$$
\operatorname{Tr}(\Phi(H+T)-\Phi(H))=\int_{-\infty}^{\infty} \Phi^{\prime}(\lambda) \xi(\lambda) d \lambda,
$$

где ф.с.с. $\xi(\lambda)$ зависит только от пары $H$ и $T$, для нее М.Г. Крейном было найдено представление

$$
\xi(\lambda)=\frac{1}{\pi} \lim _{\varepsilon \rightarrow+0} \arg D_{(H+T) / H}(\lambda+i \varepsilon)
$$

почти всюду на $\mathbb{R}$, здесь $D_{(H+T) / H}(z)$ - определитель возмущения, функция $\Phi(\lambda), \lambda \in \mathbb{R}$, имеет вид

$$
\Phi(\lambda)=\int_{z \in \mathbb{C} \backslash \mathbb{R}} \frac{d \mu(z)}{\lambda-z}, \quad \int_{z \in \mathbb{C} \backslash \mathbb{R}} \frac{|d \mu(z)|}{|\operatorname{Im} z|^{k}}<\infty, \quad k=1,2,
$$

$\mu(z)$ - вполне аддитивная комплекснозначная функция на ограниченных борелевских множествах из $\mathbb{C} \backslash \mathbb{R}$. Для $\xi(\lambda)$ верны оценка

$$
\int_{-\infty}^{\infty}|\xi(\lambda)| d \lambda \leqslant\|T\|_{1}
$$

\footnotetext{
${ }^{11}$ ниже - ф.c.c.
} 
и равенство

$$
\int_{-\infty}^{\infty} \xi(\lambda) d \lambda=\operatorname{Tr} T
$$

За (39) в дальнейшем закрепилось название формулы следа Крейна.

Прямое обобщение формулы Крейна стало одним из важных направлений развития теории следов. Формула обобщалась расширением семейства функций $\Phi(\lambda)$, с одной стороны, и расширением классов пар операторов $H$ и $T$ с другой; первоначально формула (39) не распространялась, например, на $\Phi(\lambda)=\lambda$, хотя в этом случае оператор $\Phi(H+T)-\Phi(H)$ ядерный по предположению (конечно, в силу (41) формула (39) верна и в этом случае, и подобное "распространение" делалось явочным порядком, мы здесь имеем в виду то, что функция $\Phi(\lambda)=\lambda$ не включалась естественным образом в класс функций, для которых формула (39) была строго доказана), и даже на $\Phi(\lambda)(38)$ из примера работы Лифшица. Первые обобщения сделал сам М.Г. Крейн, в той же работе предложив вариант формулы (39) в виде предела по некоторой последовательности операторов, что было абстрактным (и более аккуратным) вариантом предельного перехода по малому параметру $\alpha$ из работ Лифшица. В следующей работе М. Г. Крейна по этой теме [142] формула (39) была распространена на функции $\Phi(\lambda) \in C^{1}(\mathbb{R})$ такие, что имеет место представление

$$
\Phi^{\prime}(\lambda)=\int_{-\infty}^{\infty} e^{i \lambda t} d \omega(t), \quad \text { где } \quad \int_{-\infty}^{\infty}|d \omega(t)|<\infty .
$$

В этот класс функция $\Phi(\lambda)=\lambda$ вошла уже естественным образом. Также в работе [142] формула следа была распространена на пары унитарных операторов и далее, с помощью преобразования Кэли, на некоторые классы пар резольвентно сравнимых операторов. Конечно, ф.с.с. удовлетворяла при этом более жесткому, чем (40), условию и формула следа была верна для другого класса функций $\Phi(\lambda)$.

Вся ранняя и значительная часть дальнейшей истории исследования ф.с.с. и формулы следа Крейна хорошо изложена в обзоре 1994 года М. Ш. Бирмана и Д. Р. Яфаева [6], а в монографии [5] дано полное и строгое изложение классической теории, поэтому далее мы ограничимся кратким указанием на основные работы и обзором работ, не попавших в [6].

Сразу вслед за статьей [142] вышла работа [143], содержавшая знаменитую формулу Бирмана-Крейна, связавшую ф.с.с. с матрицей рассеяния $S(\lambda)$ (см. ниже формулу (47)) в абстрактной постановке задачи рассеяния:

$$
\operatorname{det} S(\lambda)=e^{-2 i \pi \xi(\lambda)} .
$$

Эта формула может трактоваться и как вариант формулы следа, из нее можно получить формулы Л. Д. Фаддеева и В. С. Буслаева [12], [13], [68] для задач рассеяния ДО второго порядка; также в этом контексте упомянем последний параграф уже обсуждавшейся работы И. М. Лифшица [141], в котором в качестве приложения развитой теории автор рассмотрел “колебания кристаллической решетки, испорченной находящимся в ней чужеродным атомом", и ограничимся цитатой: "Таким образом при переходе через местное искажение решетки волна ... меняет свою фазу на $2 \pi \xi "$. 
Ряд авторов вместо условий резольвентной сравнимости операторов использовали удобное в приложениях условие ядерности часто встречающегося в теории возмущений оператора $T(H-\lambda E)^{-1}$, среди первых таких работ отметим [12], [13]. Интересный вариант этого условия рассмотрел В. А. Яврян [144]: в случае ядерности оператора $(H-\lambda E)^{-1 / 2} T(H-\lambda E)^{-1 / 2}$ он ввел модифицированный определитель возмущения, определил ф.с.с. и доказал формулу следа. На основе этого результата автору удалось получить обобщение результатов работ [12], [13].

Важное продвижение сделал Л. С. Коплиенко [145], рассмотрев случай принадлежности оператора $T$ классу Гильберта-Шмидта. Использовав регуляризованный определитель возмущения, он для некоторого класса функций $\Phi(\lambda)$ доказал существование функции $\theta(\lambda) \in L_{1}(\mathbb{R})$, зависящей только от пары операторов, такой, что верна формула

$$
\operatorname{Tr}\left(\Phi(H+T)-\Phi(H)-\left.\Phi_{\varepsilon}^{\prime}(H+\varepsilon T)\right|_{\varepsilon=0}\right)=\int_{-\infty}^{+\infty} \theta(\lambda) \Phi^{\prime \prime}(\lambda) d \lambda .
$$

Позже в работе [146] формула (43) была распространена на случай резольвентной сравнимости возмущенного и невозмущенного операторов по модулю операторов Гильберта-Шмидта, еще одно интересное обобщение было получено в статье [147] с использованием другого метода - анализа операторной функции $\exp (i t(H+\varepsilon T))$ малого параметра $\varepsilon$. В этой работе для более широкого (в частности, содержащего неаналитические функции) класса функций была доказана формула (43) и, кроме того, некоторые аналогичные результаты для возмущений $T \in \mathfrak{S}_{p}, p>2$.

Глубокие результаты в теории имеются в направлении, связанном с отказом от самосопряженности или унитарности операторов. Различные аналоги ф.с.с. и формулы следов рассматривались для диссипативных операторов и сжимающих операторов в значительном количестве работ, мы ограничимся ссылкой на работу А. В. Рыбкина [148], изложение истории вопроса в которой является хорошим обзором этой части теории.

Из более поздних работ, посвященных теории ф.с.с. и ее приложениям, выделим работу А. Полторацкого [149], в которой детальное исследование ф.с.с. применено к исследованию устойчивости относительно одномерных возмущений свойства оператора иметь чисто точечный спектр, две работы А. Б. Пушницкого [150], [151], в первой из которых для ф.с.с. при неотрицательных возмущениях оператора получено новое интегральное представление, поточечные оценки и достаточное условие ее непрерывности, а во второй для возмущения оператора Шрёдингера в $\mathbb{R}^{n}$ с переменной римановой метрикой и электромагнитным полем оператором умножения на функцию $V(x)$ получены новые интегральные оценки для ф.с.с. в терминах $V(x)$. В работе [152] Е. Коротаев и А. Пушницкий получили формулы следов для возмущенного оператора Штарка. В. Мюллер [153] провел интересное исследование в теории рассеяния, использовав формулу Крейна и связь между $\zeta$-функцией и $\theta$-функцией для определения "относительной” $\zeta$-функции и глубоко исследовав на этом пути регуляризованные определители возмущения. Отметим явную перекличку этого с выше обсуждавшимся для дискретных операторов методом суммирования следов по Абелю. 
Из большого цикла работ, имеющих непосредственное отношение к обсуждаемой теме, выполненных Б. Саймоном с группой сотрудников, в обзор [6] успели попасть лишь несколько первых, 1987-88 годов, в которых формулы следа вновь нашли применение для исследования индекса, на этот раз в рамках современных математических моделей в квантовой механике, мы вернемся к ним в дополнении 3. Позже в цикле работ (см. работы [24], [154] и приведенные там дальнейшие ссылки) та же исследовательская группа обратилась к изучению ДО второго порядка, заданных на всем пространстве. Одной из центральных целей был перенос формул следа типа Левитана в периодической задаче, которая, как уже отмечалось выше, в каком-то смысле решает обратную задачу, на непериодический случай. Заметим, что по существу первый шаг на этом пути был сделан в уже упоминавшейся статье [90]. В работе [24] рассмотрен оператор

$$
H y=-y^{\prime \prime}+V(x) y,
$$

действующий в $L_{2}(-\infty,+\infty)$ с непрерывным и ограниченным снизу потенциалом, и оператор $H_{x}$, заданный тем же дифференциальным выражением и действующий в $L_{2}(-\infty, x) \oplus L_{2}(x,+\infty)$ с условием Дирихле в точке $x$. Тогда разность резольвент этих операторов одномерна и для них существует ф.c.c. $\xi(x, \lambda)$. Формула следа, доказанная в работе, имеет вид

$$
V(x)=E_{0}+\lim _{\alpha \rightarrow 0} \int_{E_{0}}^{+\infty} e^{-\alpha \lambda}(1-2 \xi(x, \lambda)) d \lambda,
$$

где $E_{0}$ - лежащее ниже спектра оператора $H$ число. Эта формула близка формулам типа Фаддеева [12] или Гасымова [10], особенно если рассматривать их на полуоси с началом в произвольной точке $x$, общность потенциала в данном случае, по-видимому, объясняет присутствие в формуле аналога суммирования по Абелю. Во всяком случае, для оператора с чисто дискретным спектром неизбежность суммирования показана в работе. Аналогичный результат получен и для бесконечной матрицы Якоби. Также в работах этой группы сотрудников Б. Саймона, а также в работах А. В. Рыбкина (см. [155] и дальнейшие ссылки) исследовались и различные (в том числе необходимые и достаточные) условия существования формул следов, их суммируемости, абсолютной сходимости.

В завершение обсуждения формулы Крейна мы остановимся на вопросе, впервые поставленном И. М. Гельфандом в 1956 году [26]: установить прямую связь формул следа типа Крейна и типа Гельфанда-Левитана. Поясним, о чем идет речь.

В формуле Крейна из двух операторов $H$ и $H+T$ конструируется один ядерный оператор $\Phi(H+T)-\Phi(H)$ и далее находится выражение для его следа, ценность которого в четком разделении вклада исходной пары операторов, определяемого их ф.с.с., и вклада самой конструкции этого ядерного оператора, определяемой функцией $\Phi(\lambda)$.

В формуле типа Гельфанда-Левитана речь всегда идет о двух операторах, никакой ядерности разности операторов не предполагается по самому смыслу задачи, а находится явное выражение для некоторой числовой характеристики - суммы разностей собственных чисел (при необходимости дополнительно регуляризованной), другими словами, геометрия действия операторов в гильбертовом пространстве игнорируется. 
Таким образом, интуитивное ожидание связи между этими формулами, подкрепляемое некоторыми конкретными примерами и формальными выкладками, при строгом взгляде входит в противоречие с явными существенными различиями в них. В силу этого печатных попыток ответить на вопрос Гельфанда почти не было, а известные нам устные ограничивались прослеживанием аналогий (подчас весьма глубоких) в конкретных примерах. Характерная же печатная попытка предложена в работе [99] на основе формулы (43). Сразу отметим, что эта формула по структуре неприемлема для класса функций $\Phi(\lambda)$, включающего в себя $\Phi(\lambda)=\lambda$, так как, сохраняя формальную справедливость и в этом случае, формула вырождается до равенства тождественных нулей. В [99] формально из (43) выведено равенство

$$
\operatorname{Tr}\left(\Phi(H+T)-\Phi(H)-\left.\Phi_{\varepsilon}^{\prime}(H+\varepsilon T)\right|_{\varepsilon=0}\right)=-\int_{-\infty}^{+\infty} \Phi^{\prime}(\lambda) d \theta(\lambda),
$$

и подстановкой $\Phi(\lambda)=\lambda$ получено (формально и при дополнительных предположениях неясного происхождения)

$$
\int_{-\infty}^{+\infty} d \theta(\lambda)=0=\sum\left(\mu_{n}-\lambda_{n}-\left(T \varphi_{n}, \varphi_{n}\right)\right)
$$

Подобные манипуляции и подгонку под известный ответ вряд ли можно считать приемлемыми. Мы выскажем свою [156] точку зрения на этот вопрос, ограничившись двумя классическими примерами - исходной формулой ГельфандаЛевитана (4) для обсуждения дискретного случая и формулой Фаддеева (13) для случая непрерывного спектра (для простоты полагая, что при возмущении дискретный спектр не появляется). Мы увидим, что обсуждаемая связь формул, с одной стороны, обладает определенным единством для операторов с произвольной природой спектра, но, с другой стороны, есть существенные различия в зависимости от того, являются рассматриваемые операторы дискретными или нет.

Итак, рассмотрим два самосопряженных дискретных оператора $A_{1}$ и $A_{2}$ с собственными числами $\left\{\lambda_{n}\right\}$ и $\left\{\mu_{n}\right\}$ соответственно и с собственными векторами $\left\{\varphi_{n}\right\}$ и $\left\{\psi_{n}\right\}$, образующими два ортонормированных базиса. Тогда существует унитарный оператор $U$, определяемый действием на базисе $U \psi_{n}=\varphi_{n}$, такой, что операторы $A_{1}$ и $U A_{2} U^{-1}$ коммутируют. Так как спектр оператора является унитарным инвариантом и так как для пары дискретных операторов ф.с.с. есть разность считающих функций $N_{i}(\lambda)$, то для ф.с.с. $\xi\left(\lambda, A_{1}, A_{2}\right)$ пары $A_{1}$ и $A_{2}$ и ф.c.c. $\xi\left(\lambda, A_{1}, U A_{2} U^{-1}\right)$ пары $A_{1}$ и $U A_{2} U^{-1}$ верно равенство

$$
\xi\left(\lambda, A_{1}, A_{2}\right)=\xi\left(\lambda, A_{1}, U A_{2} U^{-1}\right) .
$$

Это равенство позволяет для дискретных операторов дать следующее обобщение формулы Крейна, которое правильнее сформулировать в виде определения, опирающегося на классические результаты: если для пары дискретных операторов $H+T$ и $H$ существуют унитарный оператор $U$ и класс функций $\Phi(\lambda)$ такие, что оператор $\left(\Phi\left(U(H+T) U^{-1}\right)-\Phi(H)\right)$ ядерный, то регуляризованным следом пары операторов $\Phi(H+T)$ и $\Phi(H)$ называется величина

$$
\operatorname{Tr}\left(\Phi\left(U(H+T) U^{-1}\right)-\Phi(H)\right)=\int_{-\infty}^{\infty} \Phi^{\prime}(\lambda) \xi(\lambda) d \lambda,
$$


причем в силу (45) можно считать, что в (46) стоит ф.с.с. операторов $H+T$ и $H$. Применение к оператору (3) тривиально: если (как и предполагали авторы [7]) среднее потенциала на отрезке равно нулю, то $\mu_{n}=n^{2}+O\left(1 / n^{2}\right)$ и ясно (в силу абсолютной сходимости ряда из остаточных членов), что этот оператор унитарно эквивалентен оператору $-d^{2} / d x^{2}$ с теми же краевыми условиями, возмущенному ядерным оператором, и тогда формула (46) применима с функцией $\Phi(\lambda)=\lambda$, причем (в несколько вольных обозначениях)

$$
\operatorname{Tr}\left(U\left(-\frac{d^{2}}{d x^{2}}+q(x)\right) U^{-1}+\frac{d^{2}}{d x^{2}}\right)=\sum\left(\mu_{n}-n^{2}\right) .
$$

Заметим, что способ вычисления левой части до окончательного ответа неочевиден, и хотя в данном случае его, по-видимому, можно найти с помощью операторов преобразования, вряд ли он будет проще даже метода оригинальной работы [7]. Однако отметим, что работы [138], [20], [130], [129] по существу опирались именно на идею формулы (46).

Для операторов с непрерывным спектром это рассуждение не годится - хотя спектр и является унитарным инвариантом, однако ф.с.с. в этом случае таковым не является: например, если оператор второго порядка возмутить таким убывающим потенциалом, что дискретный спектр не появляется, то этот оператор унитарно эквивалентен невозмущенному, и равенство (45) не имеет места, а верно $\xi\left(\lambda, A_{1}, U A_{2} U^{-1}\right)=0$. Однако хорошо известно, что среди осуществляющих эту унитарную эквивалентность операторов можно выделить два оператора $U^{( \pm)}$, называемых волновыми операторами,

$$
U^{( \pm)}=\lim _{t \rightarrow \pm \infty} e^{i A_{2} t} e^{-i A_{1} t}
$$

таких, что оператор рассеяния $S=U^{(+) *} U^{(-)}$содержит всю информацию о ф.с.с.; собственно, в этом и состоит основной результат работы [143], см. (42). Дальнейшая связь с формулой Фаддеева хорошо известна и уже нами обсуждалась.

Таким образом, в обоих случаях связь между двумя типами формул следов осуществляется с помощью правильно примененных унитарных преобразований, и любопытна возникающая при этом "зеркальность": в дискретном случае унитарный оператор полностью сохраняет нужную информацию в самих операторах, в непрерывном, напротив, полностью уничтожает, забирая ее при этом в себя.

\section{Дополнение 2. 〈-функция, $\theta$-функция, некоммутативный вычет и спектральная асимметрия}

Три числовые функции, определеннные на спектре оператора (или на дополнении к спектру):

$\zeta$-функция оператора $A$ с собственными числами $\lambda_{n}$, определяемая равенством

$$
\zeta_{\gamma}(s)=\sum \lambda_{n}^{-s}=\operatorname{Tr} A^{-s}
$$

где $\gamma$ - аргумент луча, участвующего в выборе ветви логарифма при определении комплексных степеней чисел $\lambda_{n}$ (аналогичный смысл имеет индекс у Һ-функции (19)); 
$\theta$-функция

$$
\theta(t)=\sum e^{-t \lambda_{n}}=\operatorname{Tr} e^{-t A}
$$

и след резольвенты

$$
\rho(\lambda)=\sum\left(\lambda_{n}-\lambda\right)^{-1}
$$

используются при исследовании распределения дискретного спектра оператора очень давно, мы ограничимся ссылкой на обзор Г. Вейля [157]. Хотя исследование любого из объектов (48), (49), (50) имеет самостоятельную ценность, так как проводится различной техникой и охватывает различные классы операторов, в большинстве случаев в силу известных связей

$$
\Gamma(s) \zeta_{\gamma}(s)=\int_{0}^{+\infty} t^{s-1} \theta(t) d t, \quad \rho(\lambda)=\int_{0}^{+\infty} e^{t \lambda} \theta(t) d t
$$

достаточно знать свойства какой-либо из этих функций, и мы далее ограничимся обсуждением $\zeta$-функции.

В современной теории основополагающей работой считается статья Р. Т. Сили [158], в которой комплексные степени

$$
A_{\gamma}^{-s}=-\frac{1}{2 \pi i} \int_{\Gamma} \lambda^{-s}(A-\lambda)^{-1} d \lambda
$$

были определены для весьма широкого класса эллиптических ПДО на многообразиях без края ( $\gamma$ указывает на аргумент входящего в контур Г луча). В этой работе было детально изучено аналитическое продолжение интегрального ядра семейства операторов (51) как вне диагонали, так и на ней, и исследована $\zeta$-функция с предъявлением явных рекуррентных формул для вычетов $\zeta$-функции в полюсах и значений в целых точках при регулярности в них функции. Мы считаем необходимым отметить уже обсуждавшиеся в связи со следами работы Л. А. Дикого [33], [34] и В.А. Садовничего [42]: Л. А. Дикий построил комплексные степени оператора Штурма-Лиувилля по сути техникой ПДО задолго до ее возникновения, а В. А. Садовничий, продолжив эти исследования, распространил технику и результаты на обыкновенные ДО высоких порядков. Подчеркнем, что речь идет о краевых задачах, а в очень близкой по технике более поздней работе Р. Т. Сили хотя и охвачены операторы в частных производных, но рассмотрены задачи на многообразиях без края. Также, безусловно, необходимо отметить в контексте теории $\zeta$-функций работу В. С. Буслаева и Л. Д. Фаддеева [13], в которой впервые $\zeta$-функция была построена для оператора с непрерывным спектром.

Дальнейший путь развития теории во многом был определен этой работой Сили, отметим здесь монографию М. А. Шубина [159], в которой можно найти содержательную библиографию и ее обсуждение, цикл работ М.Ф. Атьи, В.К. Патоди и И. М. Зингера (см. завершающую работу цикла [160]), П.Б. Джилки [161]. Выделим здесь введенное В.А. Садовничим понятие взвешенной $\zeta$-функции [52], которая может нести в себе всю информацию об операторе (см., например, [116]).

Часть авторов в исследованиях $\zeta$-функции опиралась на теорию некоммутативного вычета. Дадим его определение. Пусть $A$ - классический ПДО, 
действующий в сечениях векторного расслоения на гладком замкнутом многообразии $M$ размерности $n, a(x, \xi) \sim \sum_{j=0}^{\infty} a_{m-j}(x, \xi)$ - его полный символ ${ }^{12}$, и тогда в случае, если среди чисел $m-j$ есть число $-n$, определим на $M$ плотность

$$
\operatorname{res}(x ; A)=\left(\int_{|\xi|=1} a_{-n}(x, \xi) d S_{\xi}\right) d x,
$$

в ином случае положим $\operatorname{res}(x ; A) \equiv 0$. Некоммутативным вычетом оператора $A$ называется число

$$
\operatorname{res} A=\int_{M} \operatorname{Tr} \operatorname{res}(x ; A) .
$$

Отсылая читателя за подробностями и дальнейшими ссылками к работе М. Водзицкого [162], отметим, что понятие и свойства некоммутативного вычета позволяют строить аналитическое продолжение интегральных ядер некоторых типов голоморфных оператор-функций, в частности, комплексных степеней операторов и, более общо, операторов вида $B A^{-s}$. Связанная с этой операторфункцией обобщенная $\zeta$-функция $\operatorname{Tr}\left(B A^{-s}\right)$ имеет ряд приложений в квантовой теории поля, спектральной геометрии, теории чисел, а соответствующий некоммутативный вычет прямо связан со следующей нашей темой - спектральной асимметрией.

Спектральной асимметрией называют зависимость $\zeta$-функции (48) от выбора параметра $\gamma$. Основной предмет изучения - те характеристики $\zeta$-функции, которые от этого параметра не зависят либо их зависимость явно вычисляется. Для широкого класса ПДО известно, что функция

$$
\zeta_{\gamma^{\prime}}(s)-\zeta_{\gamma^{\prime \prime}}(s)
$$

регулярна в целых точках, при этом ее значение в нуле всегда нуль. Последнее обстоятельство весьма существенно в теории индекса, к чему мы вернемся в следующем дополнении. Считается, что на регулярность разности двух $\zeta$-функций впервые обратили внимание Атья, Патоди и Зингер в цикле работ 1973-1976 годов, завершенном работой [160], в частности, они детально изучившие в самосопряженном неполуограниченном случае функцию

$$
\eta(z)=\sum\left|\lambda_{n}\right|^{z} \operatorname{sign} \lambda_{n}
$$

однако отметим, что эти же вопросы ранее рассматривал В.А. Садовничий [48], [163].

Вообще, именно теория $\zeta$-функции, ассоциированной с функцией класса $K$, фактически положила начало и теории спектральной асимметрии, и это обстоятельство незаслуженно забыто. В работе [11] $\zeta$-функция, ассоциированная с целой функцией класса $K$, была целой, что стало для специалистов изрядным сюрпризом и некоторое время этот результат сохранял статус "странного". В указанных работах В.А. Садовничий обнаружил (см. подробнее в настоящем обзоре стр. 106), что $\zeta$-функция, построенная на последовательности части корней функции класса $K(18)$ из одного сектора, является мероморфной

\footnotetext{
${ }^{12}$ Строго говоря, надо добавить: “в какой-либо карте”, но мы будем пренебрегать загромождающей строгостью, которую легко найти во многих хорошо известных монографиях.
} 
и что исходная целая $\zeta$-функция является линейной комбинацией таких мероморфных. Заметим, что функция (52) как раз имеет структуру линейной комбинации двух обычных $\zeta$-функций. Укажем в этой связи и на работу [54], в которой рассмотрена $\zeta$-функция вида $Z(\sigma)=\sum \lambda_{n}^{-\sigma} \Lambda(\sigma)$, где

$$
\Lambda(\sigma)= \begin{cases}h, & \sigma=0, \pm 1, \pm 2, \ldots, \\ \frac{e^{-2 \pi h \sigma i}-1}{e^{-2 \pi \sigma i}-1} & \text { в других точках. }\end{cases}
$$

В заключение укажем, что мы полностью пропускаем обсуждение теории бесконечных определителей и их регуляризации, ограничиваясь ссылкой на одно весьма общее современное исследование - работу [164], и имеющуюся там литературу, и также не обсуждаем широко распространенное в физической литературе использование значений $\zeta$-функций как следа оператора без регуляризации, см., например, [121].

\section{Дополнение 3. Теория индекса линейных операторов}

Теория индекса является одной из самых известных задач теории операторов второй половины 20-го века, она многократно и хорошо изложена, и мы ограничимся короткими указаниями на теснейшую и взаимополезную связь теории регуляризованных следов с теорией индекса.

Одна из известных формул для индекса фредгольмова оператора $A$

$$
\operatorname{ind}(A)=\operatorname{Tr} e^{-t A^{*} A}-\operatorname{Tr} e^{-t A A^{*}}
$$

позволяет исследовать индекс с помощью следов. Естественно, (53) не зависит от $t$ и обладает свойством “топологической инвариантности": $\operatorname{ind}(A+B)=$ $\operatorname{ind}(A)$ при компактном операторе $B$. Аналогичные формулы, в которых вместо $\theta$-функций используются $\zeta$-функции, широко исследовались и до появления работы Р. Т. Сили [158], а после этой работы такой метод в отношении ПДО стал главенствующим. Уже упоминавшиеся в предыдущем дополнении работы М. Ф. Атья, В. К. Патоди и И. М. Зингера являются одним из крупнейших примеров исследований такого рода. Многочисленные применения именно такого подхода проведены в серии работ Б. В. Федосова (см. [165], [166] и приведенные там дополнительные ссылки), охватившего в том числе и многие краевые задачи, и в работах Б.В. Федосова и М.А. Шубина о случайных операторах (см. [167]).

Прямо сформулированное приложение теории регуляризованных следов в теории индекса эллиптических операторов дано в монографии Ш. Ремпеля и Б.-В. Шульце [168], в ней для широкого класса ПДО $A$ на многообразиях с краем регуляризованный след введен следующим равенством: $\operatorname{Tr}(A-\operatorname{Op}(\sigma(A)))$, где $\sigma(A)$ - символ оператора $A$. Доказана важная для корректности определения теорема о независимости регуляризованного следа композиции операторов от порядка сомножителей и далее регуляризованный след использован для формулировки и доказательства ряда формул для индекса операторов.

В работах Ф. Гештези, Б. Саймона и других [169], [170] исследовался регуляризованный индекс Виттена

$$
\operatorname{ind}_{t}(A)=\operatorname{Tr}\left(e^{-t A^{*} A}-e^{-t A A^{*}}\right)
$$


являющийся естественным обобщением (стандартной регуляризацией следа в духе (5)) формулы (53). В качестве инструмента использовалась формула Крейна и было получено представление

$$
\operatorname{ind}_{t}(A)=-t \int_{0}^{\infty} e^{-t \lambda} \xi(\lambda) d \lambda
$$

и тогда для самого индекса Виттена, определяемого как

$$
\lim _{t \rightarrow \infty} \operatorname{ind}_{t}(A)=W(A),
$$

были получены следующие две формулы:

$$
W(A)=-\xi(0+)
$$

и в случае резольвентной сравнимости операторов $A A^{*}$ и $A^{*} A$

$$
W(A)=\lim _{\tau \rightarrow 0+} \tau \operatorname{Tr}\left(\left(A^{*} A+\tau\right)^{-1}-\left(A A^{*}+\tau\right)^{-1}\right) .
$$

Нетрудно видеть, имея в виду, что для дискретных операторов ф.с.с. есть разность считающих функций спектра, что (55) для дискретных операторов есть в точности дефект регуляризации (22) (конечно, вычисляемый по формуле (22) только в случае применимости метода функций класса $K$ ).

Также в [170] была доказана топологическая инвариантность регуляризованного индекса Виттена $\operatorname{ind}_{t}(A+B)=\operatorname{ind}_{t}(A)$ для операторов $B$ таких, что для некоторого $\varepsilon<1 / 2$ операторы $B\left(A^{*} A+1\right)^{-\varepsilon}$ и $B^{*}\left(A A^{*}+1\right)^{-\varepsilon}$ оба ограничены и для некоторого $\delta>0$ операторы $B\left(A^{*} A+1\right)^{-\delta}$ и $B^{*}\left(A A^{*}+1\right)^{-\delta}$ оба ядерные. В работе приведены и более точные условия, также отметим, что библиография к этой работе содержит интересные ссылки по приложениям формулы Крейна.

\section{Дополнение 4. О приближенном вычислении первых собственных чисел}

В 1957 году И. М. Гельфанд и Л. А. Дикий предложили (см. [171]) использовать регуляризованные следы для приближенного вычисления собственных чисел операторов. Расположение первых собственных чисел отвечает за устойчивость многих динамических систем, и поэтому актуален любой метод исследования этого вопроса. Сходящиеся ряды из отрицательных степеней собственных чисел использовались ранее для вычисления отдельных слагаемых (см., например, [172]), однако трудность вычисления следов итерированных функций Грина сильно проигрывала простоте и точности выражения через параметры оператора регуляризованных следов, что послужило дополнительным стимулом к постановке данной задачи.

Суть выдвинутой гипотезы в следующем: авторы предположили, что всегда возможно, располагая полной системой регуляризованных следов оператора (в данном случае Штурма-Лиувилля)

$$
\sum_{n=1}^{\infty}\left(\mu_{n}^{k}-A_{k}(n)\right)=B(k), \quad k=1, \ldots, \infty
$$


для заданного $\varepsilon>0$ найти такое $N$, что система

$$
\sum_{n=1}^{N}\left(\mu_{n}^{k}-A_{k}(n)\right)=B(k), \quad k=1, \ldots, N
$$

определяет несколько первых собственных чисел с точностью $\varepsilon$. В работе [171] эта идея была без обоснования реализована для первых трех собственных чисел оператора Матье и было получено вполне удовлетворительное совпадение найденных величин с табличными, однако ни тогда, ни позже обосновать этот метод не удалось. Более того, в 1996 году С.А. Шкарин [173] доказал, что системы уравнений, подобные (56), имеют бесконечно много решений, и в частности, в них можно заранее задавать любое конечное множество $\mu_{n}-\mathrm{x}$, и найдется соответствующее решение всей системы ${ }^{13}$. Подобное обстоятельство делает рассмотрение систем типа (57) в общем случае просто бессмысленным. Тем не менее были найдены другие подходы к этой задаче. В.А. Садовничий и В. Е. Подольский [175] ввели специальный класс $S$ операторов ШтурмаЛиувилля

$$
-y^{\prime \prime}+q(x) y=\lambda y, y^{\prime}(0)-h y(0)=0, \quad y^{\prime}(\pi)+H y(\pi)=0
$$

с $q(x) \in L_{2}[0, \pi], h, H \in \mathbb{R}$, характеризуемый через свойства $\varphi(x, \lambda)$ - решения задачи Коши с начальными данными $\varphi(0, \lambda)=1, \varphi^{\prime}(0, \lambda)=h$. Если $q(x) \in C^{n}[0, \pi]$, то хорошо известно, что $\varphi(x, \lambda)$ при $|\lambda| \rightarrow \infty$ имеет следующее асимптотическое разложение:

$$
\varphi(x, \lambda)=\cos \sqrt{\lambda} x+\cdots+k_{2 i}(x) \frac{\cos \sqrt{\lambda} x}{(\sqrt{\lambda})^{2 i}}+k_{2 i+1}(x) \frac{\sin \sqrt{\lambda} x}{(\sqrt{\lambda})^{2 i}}+\cdots+O\left(\frac{e^{|\operatorname{Im} \sqrt{\lambda}| \pi}}{|\lambda|^{\frac{n+1}{2}}}\right),
$$

где $k_{i}(x)$ рекуррентно определяются потенциалом и параметром $h$. Класс $S$ характеризуется тем, что в этом разложении для некоторого целого $j, 1 \leqslant$ $j \leqslant n+1$, коэффициент $k_{j}(x) \equiv 0$ на $[0, \pi]$. Оказывается, потенциал $q(x)$ любого оператора класса $S$ аналитически продолжается во всю плоскость как мероморфная функция с полюсами второго порядка, ядро оператора преобразования $K(x, t)$ для операторов класса $S$ есть полином по второй переменной, переходная функция обратной задачи $\Phi(x)$ есть обязательно квазиполином и, более того, любой полином по четным степеням $x$ является переходной функцией обратной задачи для некоторого оператора класса $S$. В этой работе было доказано, что система (56) однозначно определяет спектр оператора класса $S$ и, более того, для любого $\varepsilon>0$ существует такое натуральное $N(\varepsilon)$, а также существует такое натуральное $K$, зависящее только от оператора $L$ (и не зависящее от $\varepsilon)$, что если использовать в $A_{n}(k)$ при $k=1, \ldots, K$ первые $N(\varepsilon)$ членов асимптотического разложения $\lambda_{n}$ по степеням $n$, то будут верны неравенства

$$
\left|\sum_{n=1}^{K}\left(\lambda_{n}^{k}-A_{n}(k)\right)-B(k)\right| \leqslant \varepsilon, \quad k=1, \ldots, K,
$$

\footnotetext{
${ }^{13}$ Недавно мы повторили и прояснили результат Шкарина в рамках теории функций класca $K$ [174].
} 
причем в работах даны все необходимые конструктивные оценки. И второй важный результат этих работ, распространяющий возможность вычислять собственные числа оператора через следы на "все" операторы Штурма-Лиувилля, состоит в доказательстве того, что для произвольного оператора ШтурмаЛиувилля $L$ с потенциалом из $L_{2}[0, \pi]$ и произвольного $\varepsilon>0$ существует оператор класса $S$ с такой же областью определения, как и у $L$, и такой, что норма их разности не превосходит $\varepsilon$. Эти результаты были использованы в работе [176].

Вполне естественно, что формулы следов для абстрактных операторов, являющиеся частью теории возмущений спектра операторов, приводят к новым методам приближенного вычисления собственных чисел. Такой метод был предложен В.А. Садовничим и В.В.Дубровским в 1994 году в статье [177]. Суть метода в следующем: в силу формул теории возмущений для собственных чисел $\mu_{n}$ оператора $A+B$ и собственных чисел $\lambda_{n}$ оператора $A$ верны равенства (предполагается, что существует система контуров $\Gamma_{m}$, внутри которых количество собственных чисел операторов одинаково):

$$
\sum_{n=1}^{n_{m}} \mu_{n}^{k}=\sum_{n=1}^{n_{m}} \lambda_{n}^{k}+\sum_{l=1}^{p_{k}} \alpha_{l k}\left(n_{m}\right)+\beta_{k p_{k}}\left(n_{m}\right),
$$

где

$$
\alpha_{l k}\left(n_{m}\right)=\frac{(-1)^{l+1} k}{2 \pi i l} \operatorname{Tr} \int_{\Gamma_{m}} \lambda^{k-1}(B R(A, \lambda))^{l} d \lambda
$$

- $l$-я поправка теории возмущений для $k$-й степени оператора для первых $n_{m}$ собственных чисел, $\beta_{k p_{k}}\left(n_{m}\right)$ - сумма остальных поправок, ряд из которых заведомо сходится, если $p_{k}$-я степень резольвенты оператора будет ядерной. Теперь, если величины $\beta_{k p_{k}}\left(n_{m}\right)$ окажутся достаточно малы, то система (58) определит $\mu_{n}$ с высокой точностью.

Метод работы [177] удалось применить к некоторым важны задачам математической физики. В [178] этим методом проведено вычисление первых собственных чисел в задаче гидродинамической устойчивости течения Пуазейля в круглой трубе. Данная задача имеет вид

$$
T^{2} \varphi+U \varphi=\operatorname{R\sigma rT} \varphi, \quad 0 \leqslant r \leqslant 1, \quad \varphi(1)=\left.\frac{d \varphi}{d r}\right|_{r=1}=0, \quad|\varphi(0)|<\mathrm{const},
$$

где

$$
T \varphi=\left(r \frac{d^{2}}{d r^{2}}+\frac{d}{d r}-\frac{1}{r}-\lambda^{2} r\right) \varphi, \quad U \varphi=-i \lambda R r\left(1-r^{2}\right) T \varphi,
$$

$\sigma$ - спектральный параметр, $\lambda$ - волновое число, $R$ - число Рейнольдса. Применением метода работы [177] к задаче, получаемой из исходной умножением на $T^{-1}$, удалось получить систему

$$
\sum_{i=1}^{4} \sigma_{i}^{k} \approx \sum_{i=1}^{4} \mu_{i}^{k}+\sum_{i=1}^{4} \alpha_{i}^{k}
$$

с точными оценками приближения, где $k=1, \ldots, 4, \alpha_{i}$ - поправки теории возмущений, а $\mu_{i}$ - собственные числа невозмущенной задачи

$$
T \varphi=\operatorname{R\mu r} \varphi, T^{-1} \varphi(1)=\left.\frac{d T^{-1} \varphi}{d r}\right|_{r=1}=0 .
$$


Приведем некоторые численные результаты решения этой системы:

при $R=1 \quad \mu_{1}=-2.64 \cdot 10^{1}, \sigma_{1}=\mu_{1}-2.79 \cdot 10^{-2} i, \mu_{2}=-7.09 \cdot 10^{1}, \sigma_{2}=$ $\mu_{2}-2.63 \cdot 10^{-2} i, \mu_{3}=-1.35 \cdot 10^{2}, \sigma_{3}=\mu_{3}-2.58 \cdot 10^{-2} i, \mu_{4}=-2.19 \cdot 10^{2}$, $\sigma_{4}=\mu_{4}-2.55 \cdot 10^{-2} i$;

при $R=5 \quad \mu_{1}=-5.28 \cdot 10^{0}, \sigma_{1}=\mu_{1}-1.40 \cdot 10^{-1} i, \mu_{2}=-1.42 \cdot 10^{1}, \sigma_{2}=$ $\mu_{2}-1.31 \cdot 10^{-1} i, \mu_{3}=-2.70 \cdot 10^{1}, \sigma_{3}=\mu_{3}-1.29 \cdot 10^{-1} i, \mu_{4}=-4.38 \cdot 10^{1}$, $\sigma_{4}=\mu_{4}-1.28 \cdot 10^{-1} i$

при $R=10 \quad \mu_{1}=-2.64 \cdot 10^{0}, \sigma_{1}=\mu_{1}-2.81 \cdot 10^{-1} i, \mu_{2}=-7.09 \cdot 10^{0}$, $\sigma_{2}=\mu_{2}-2.59 \cdot 10^{-1} i, \mu_{3}=-1.35 \cdot 10^{1}, \sigma_{3}=\mu_{3}-2.60 \cdot 10^{-1} i, \mu_{4}=-2.19 \cdot 10^{1}$, $\sigma_{4}=\mu_{4}-2.55 \cdot 10^{-1} i$.

Аналогичные результаты были получены и в задаче Куэтта [179].

\section{Дополнение 5. Некоторые другие результаты}

В работе [21] П. Лакс предложил подход к вычислению формул следов, основанный на замене пары операторов (возмущенного и невозмущенного) параметрическим семейством операторов, гладко соединяющим эту пару:

$$
L(t) w_{n}(t)=\lambda_{n}(t) w_{n}(t), \quad t \in[0,1],
$$

и исследовании этой задачи с помощью дифференцирования по параметру с использованием соотношения $\left(\dot{w}_{n}(t), w_{m}(t)\right)+\left(w_{n}(t), \dot{w}_{m}(t)\right)=0$. Этот метод П. Лакс применил к целой серии задач для ДО второго порядка, возмущенных оператором умножения на функцию:

$$
L(t)=-\Delta+q(x, t)
$$

действующих в $L_{2}$ на кубе $[0,2 \pi]^{k}$. Отправляясь от сравнительно несложного равенства

$$
\int_{0}^{1} \int_{[0,2 \pi]^{k}} \dot{q}(x, t)\left(w_{n}^{2}(x, t)-M\right) d x d t=\lambda_{n}(1)-\lambda_{n}(0),
$$

в котором при выполнении наложенного автором условия независимости от времени среднего от потенциала, а именно условие

$$
\int_{[0,2 \pi]^{k}} \dot{q}(x, t) d x=0,
$$

константу $M$ можно выбрать произвольно, он пришел к формальному соотношению ${ }^{14}$

$$
\int_{0}^{1} \int_{[0,2 \pi]^{k}} \dot{q}(x, t)\left(\sum_{n}\left(w_{n}^{2}(x, t)-M\right)\right) d x d t=\sum_{n}\left(\lambda_{n}(1)-\lambda_{n}(0)\right) .
$$

\footnotetext{
${ }^{14}$ Рассуждения этой работы (и на это прямо указывает сам автор) формальны, автор в примерах ограничивается задачами, в которых возможность строгого обоснования в рамках классической теории обобщенных функций очевидна. К сожалению, мы знакомы с попытками использовать формулу (60) без обоснования в значительно более сложных ситуациях. В качестве ограничительного примера приведем формулу следа для оператора Лапласа на сфере (36): нам представляется невозможным получить верный ответ из (60) непосредственHO.
} 
Если ряд под интегралом сходится в смысле теории обобщенных функций ${ }^{15}$ и сходится $^{16}$ ряд справа, то формула (60) становится источником разнообразных формул следа. Ключевым здесь является следующее наблюдение: функция

$$
S(x)=\sum_{n}\left(w_{n}^{2}(x, t)-M\right)
$$

не зависит от $t$. Таким образом, формула (60) приобретает вид

$$
\int_{[0,2 \pi]^{k}}(q(x, 1)-q(x, 0)) S(x) d x=\sum_{n}\left(\lambda_{n}(1)-\lambda_{n}(0)\right),
$$

и остается найти более удобный вид для функции $S(x)$ в конкретных задачах. Далее в работе П. Лакс вычислил своим методом ряд хорошо известных формул и несколько новых интересных комбинаций формул следов для ДО с одинаковым дифференциальным выражением, но различными краевыми условиями. Формализм этих действий, на наш взгляд, чересчур велик, и мы не рискнем воспроизводить полученные здесь формулы (хотя и вполне изящные).

В работе [180] для дискретного оператора Штурма-Лиувилля

$$
(L u)_{n}=a_{n+1} u_{n+1}+b_{n} u_{n}+a_{n} u_{n-1}, \quad n \in \mathbb{Z}_{+}, \quad u_{-1}=0,
$$

действующего в пространстве $l_{2}$, при условиях

$$
\lim _{n \rightarrow \infty} a_{n}=\frac{1}{2}, \quad \lim _{n \rightarrow \infty} b_{n}=0, \quad \sum_{n=0}^{\infty}\left(\left|a_{n}-a_{n+1}\right|+\left|b_{n}-b_{n+1}\right|\right)<\infty
$$

получена формула

$$
\sum_{n=0}^{\infty}\left[\left(a_{n+1}^{2}-a_{n}^{2}\right) p_{n}^{2}(\lambda)+a_{n+1}\left(b_{n+1}-b_{n}\right) p_{n}(\lambda) p_{n+1}(\lambda)\right]=\frac{\sqrt{1-\lambda^{2}}}{2 \pi \mu^{\prime}(\lambda)}
$$

где $p_{n}(\lambda)$ - ортогональные по мере $\mu(\lambda), \operatorname{supp} \mu=[-1,1]$ многочлены, заданные рекуррентным соотношением $\lambda p_{n}(\lambda)=a_{n+1} p_{n+1}(\lambda)+b_{n} p_{n}(\lambda)+a_{n} p_{n-1}(\lambda)$, где $n \in \mathbb{Z}_{+}, p_{-1}(\lambda)=0, p_{0}(\lambda)=1$. В работе [181] получены аналогичные формулы для некоторых классов асимптотически $N$-периодической якобиевой матрицы. Заканчивая короткий экскурс в приложения теории следов для якобиевых матриц, укажем на недавнюю весьма глубокую работу Р. Киллипа и Б. Саймона [182], в которой формулы типа формул следов содержательно использованы для решения ряда вопросов спектральной теории.

В работе Р.Ш. Бакурадзе и И. В. Станкевича [183] были получены необычные тождества для собственных чисел и собственных функций оператора четвертого порядка

$$
y^{(4)}+q(x) y=\lambda y, \quad y(0)=y(\pi)=y^{\prime \prime}(0)=y^{\prime \prime}(\pi)=0 .
$$

\footnotetext{
${ }^{15}$ Перемена порядков суммирования и интегрирования может стать самым тонким и трудным местом в обоснованиях.

${ }^{16}$ Может быть важен характер сходимости, наличие лишь условной сходимости может оказаться недостаточным.
} 
Для функции

$$
\rho_{M, s}(x)=\sum_{k \in M} \lambda_{k}^{s} \varphi_{k}^{2}(x),
$$

где $\varphi_{k}(x)$ - ортонормированные собственные функции оператора, $\lambda_{k}$ - соответствующие собственные числа, $s \in \mathbb{N} \cup\{0\}, M$ - произвольное конечное подмножество натурального ряда, при условии $q(x) \in C^{\infty}$ были доказаны рекуррентные соотношения

$$
\rho_{M, s+2}(x)=R_{0} \rho_{M, s}(x)+R_{1} \rho_{M, s+1}(x), \quad s=0,1,2, \ldots,
$$

где $R_{0}$ и $R_{1}$ - линейные интегро-дифференциальные операторы, для которых найдены явные выражения. Заметим, что интегрированием по $x$ из этих равенств можно получить тождества только для собственных чисел. Также в работе есть ссылка на предыдущую (труднодоступную) работу авторов, в которой аналогичная задача решена для оператора второго порядка.

\section{Дополнение 6. Некоторые нерешенные задачи}

В этом дополнении мы предлагаем несколько задач, наиболее актуальных, на наш взгляд, на настоящий момент. Конечно, наша точка зрения не бесспорна, задачи выбраны нами с учетом наших собственных научных вкусов, и большая их часть относится к следам дискретных операторов. Мы сознательно не стали давать четкие и строгие постановки, это скорее качественное описание пока еще неразработанных направлений исследований.

1. Формально верная для непрерывных потенциалов формула (9) на самом деле для любого непрерывного потенциала верной не является: как мы знаем, здесь все определяется сходимостью ряда Фурье потенциала. Этот эффект близкий родственник факта существования неядерного интегрального оператора с непрерывным ядром. Для интегральных операторов найдены различные достаточные условия ядерности и более общие, чем интеграл от диагонали ядра, формулы для следа, см., например, [1]. Было бы интересно найти аналоги для формул следов (для оператора Штурма-Лиувилля значительным продвижением в этом направлении можно считать результаты работ [30], [32], но, например, для оператора Лапласа на сфере вопрос полностью открыт).

2. Представляется важным найти решение давно стоящей задачи о получении формулы первого следа для оператора Лапласа в двумерной области, конкретный "спортивный” интерес давно вызывает наиболее простая задача на квадрате ("простая" только в том смысле, что для данной области точно известна и хорошо изучена в теории чисел последовательность собственных чисел невозмущенного оператора).

3. В качестве невозмущенного рассмотрим оператор, задаваемый дифференциальным выражением $-y^{\prime \prime}+q(x) y$ с $q(x)>x^{2+\delta}$ для всех больших $|x|$ и некоторым $\delta>0$, действующий в $L_{2}(\mathbb{R})$ или в $L_{2}[0 ;+\infty)$ с каким-либо условием на границе, а в качестве возмущения - оператор умножения на ограниченную измеримую функцию $p(x)$. Наложенное условие на потенциал гарантирует не только дискретность спектра, но и ядерность резольвенты оператора, и в этом 
случае в силу результатов [17] верна формула

$$
\sum_{n=1}^{\infty}\left(\mu_{n}-\lambda_{n}-\int_{\mathbb{R}} p(x) \varphi_{n}^{2}(x) d x\right)=0,
$$

где $\varphi_{n}(x)$ - собственные функции невозмущенного оператора. Эта формула является новым результатом в весьма обширных исследованиях операторов второго порядка на неограниченных интервалах, так как вообще не было формул следов с неубывающими возмущающими потенциалами. Нам представляется интересным вопрос о дальнейшей расшифровке стоящего в ней интеграла (хотя эта задача частично выходит за рамки спектральной теории).

4. Практически не изучен оператор второго порядка на полуоси или всей оси с неограниченным снизу потенциалом. Представляется важным получение формул следов в этом случае.

5. При всем богатстве уже полученных формул следов для самых разных ДО до сих пор остается неясным ответ в случае возмущения одномерного гармонического осциллятора оператором умножения на ограниченную, но не стремящуюся к конечному пределу функцию. Ясно, пожалуй, только то, что этот ответ различен для случаев (почти) периодического возмущения и возмущения, далекого от свойств периодичности в любом, даже самом слабом смысле.

6. Укажем на интересную задачу для оператора в многомерной неограниченной области, задаваемого дифференциальным выражением гармонического осциллятора и возмущенного хотя бы финитным потенциалом. Как входят в ответ характеристики области и какие это характеристики?

7. Касаясь ДО на компактных многообразиях, укажем на задачу для оператора Лапласа-Бельтрами (или Лапласа-Бохнера) на многобразиях более чем с одной ручкой. Как количество ручек входит в ответ? Эта задача тесно примыкает к предмету спектральной геометрии, и подчеркнем, что, к сожалению, нельзя облегчить себе жизнь рассмотрением степени оператора, ведь в силу известных абстрактных теорем при ядерности резольвенты оператора эта характеристика уйдет из ответа (в следы более высоких порядков, что в данном контексте равносильно понижению степени оператора). Эта задача не подразумевает возмущения оператором умножения на функцию (хотя, разумеется, такая постановка тоже интересна), здесь имеется в виду регуляризация с помощью собственных чисел оператора Лапласа-Бельтрами на "более простом" многообразии.

8. Представляется важным получить результаты по теории следов трехдиагональных и, более общо, $(2 n+1)$-диагональных матриц. Пока еще мало изучены следы случайных операторов, причем здесь важна соответствующая трактовка получаемых ответов. В спектральной геометрии в основном используются нерегуляризованные следы - значения $\zeta$-функций либо коэффициенты асимптотики $\theta$-функций, в то время как в других приложениях правильно подобранная регуляризация снимает некоторые "наносные" характеристики, выделяя существенное (например, формулы следов в рамках метода обратной задачи). Полученные результаты теории следов в статистической физике, на наш взгляд, пока обрывочны и фрагментарны, а представляется совершенно ясным, что следы, как некие средние, должны играть в таких теориях фундаментальную роль. 


\section{Список литературы}

[1] И. Ц. Гохберг, М. Г. Крейн, Введение в теорию линейных несамосопряженных операторов в гилъбертовом пространстве, Наука, М., 1965.

[2] В.Б. Лидский, "Несамосопряженные операторы, имеющие след", Докл. АН СССР, 125:3 (1959), 485-487.

[3] И. М. Лифшиц, "Об одной задаче теории возмущений, связанной с квантовой статистикой", УМH, 7:1 (1952), 171-180.

[4] М. Г. Крейн, "О формуле следов в теории возмущений”, Матем. сб., 33(75):3 (1953), 597-626.

[5] Д. Р. Яфаев, Математическая теория рассеяния, Изд-во С.-Петербургского унта, СПб., 1994.

[6] М.Ш. Бирман, Д.Р. Яфаев, “Функция спектрального сдвига. Работы М. Г. Крейна и их дальнейшее развитие”, Алгебра и анализ, 4:5 (1992), 1-44.

[7] И. М. Гельфанд, Б. М. Левитан, "Об одном простом тождестве для собственных значений дифференциального оператора второго порядка", Докл. АН CCCP, 88:4 (1953), 593-596.

[8] Л.А. Дикий, “Об одной формуле Гельфанда-Левитана”, УМН, 8:2 (1953), 119123.

[9] R. C. Gilbert, V. A. Kramer, "Trace formulas for powers of a Sturm-Liouville operator", Canad. J. Math., 16:4 (1964), 412-422.

[10] М. Г. Гасымов, "О сумме разностей собственных значений двух самосопряженных операторов", Докл. АН СССР, 150:6 (1963), 1202-1205.

[11] В.Б. Лидский, В.А. Садовничий, "Регуляризованные суммы корней одного класса целых функций”, Функи. анализ и его прилож., 1:2 (1967), 52-59.

[12] Л. Д. Фаддеев, “О выражении для следа разности двух сингулярных дифференциальных операторов типа Штурма-Лиувилля”, Докл. АН СCCP, 115:5 (1957), 878-881.

[13] В. С. Буслаев, Л. Д. Фаддеев, “О формулах следов для дифференциального сингулярного оператора Штурма-Лиувилля”, Докл. АН ССCP, 132:1 (1960), 13-16.

[14] В. Е. Захаров, Л. Д. Фаддеев, "Уравнение Кортевега-де Фриса - вполне интегрируемая гамильтонова система", Функи. анализ и его прилож., 5:4 (1971), $18-27$.

[15] В.А. Садовничий, В.В. Дубровский, "Об одной абстрактной теореме теории возмущений, о формулах регуляризованных следов и о $\zeta$-функции операторов", Дифферени. уравнения, 13:7 (1977), 1264-1271.

[16] В. А. Садовничий, В.В.Дубровский, "О некоторых соотношениях для собственных чисел дискретных операторов. Формулы следов для дифференциальных операторов в частных производных", Дифферени. уравнения, 13:11 (1977), 20332042.

[17] В.А. Садовничий, В.Е. Подольский, "Следы операторов с относительно компактным возмущением", Матем. сб., 193:2 (2002), 129-152.

[18] В. А. Садовничий, В.В.Дубровский, "О классической формуле первого регуляризованного следа оператора Лапласа с нечетным потенциалом на сфере”, $T p$. сем. им. И.Г. Петровского, 19 (1996), 37-72.

[19] В.Е. Подольский, "Формула регуляризованного следа оператора ЛапласаБельтрами с нечетным потенциалом на сфере $S^{2}$ ", Матем. заметки, 56:1 (1994), $71-77$.

[20] V.E. Podol'skii, "On the summability of regularized sums of eigenvalues of the Laplace-Beltrami operator with potential on symmetric spaces of rank one", Russian J. Math. Phys., 4:1 (1996), 123-130. 
[21] P. D. Lax, "Trace formulas for the Schrödinger operator", Comm. Pure Appl. Math., 47:4 (1994), 503-512.

[22] F. Gesztesy, H. Holden, B. Simon, Z. Zhao, "A trace formula for multidimensional Schrödinger operators", J. Funct. Anal., 141:2 (1996), 449-465.

[23] В.А. Любишкин, В.Е. Подольский, "О суммируемости регуляризованных следов дифференциальных операторов”, Матем. заметки, 54:2 (1993), 33-38.

[24] F. Gesztesy, H. Holden, B. Simon, Z. Zhao, "Trace formulae and inverse spectral theory for Schrödinger operators", Bull. Amer. Math. Soc. (N.S.), 29:2 (1993), 250255.

[25] А. Б. Венков, "Спектральная теория автоморфных функций, $\zeta$-функция Сельберга и некоторые проблемы аналитической теории чисел и математической физики", УМН, 34:3 (1979), 69-135.

[26] И. М. Гельфанд, "О тождествах для собственных значений дифференциального оператора второго порядка", УМН, 11:1 (1956), 191-198.

[27] В.А. Марченко, Операторь Штурма-Лиувилля и их приложения, Наукова думка, Киев, 1977.

[28] В.В. Дубровский, "Регуляризованный след оператора Штурма-Лиувилля", Дифферени. уравнения, 16:6 (1980), 1127-1129.

[29] В.А. Винокуров, В.А. Садовничий, "Собственное значение и след оператора Штурма-Лиувилля как дифференцируемые функции суммируемого потенциала", Докл. РАН, 365:3 (1999), 295-297.

[30] В. А. Винокуров, В. А. Садовничий, "Асимптотика собственных значений и собственных функций и формула следа для потенциала, содержащего $\delta$-функции”, Докл. РАН, 376:4 (2001), 445-448.

[31] А. М. Савчук, "Регуляризованный след первого порядка оператора ШтурмаЛиувилля с $\delta$-потенциалом", УМН, 55:6 (2000), 155-156.

[32] А. М. Савчук, А.А. Шкаликов, "Формула следа для операторов ШтурмаЛиувилля с сингулярными потенциалами", Матем. заметки, 69:3 (2001), 427442.

[33] Л. А. Дикий, “Дзета-функция обыкновенного дифференциального уравнения на конечном отрезке”, Изв. АН СССР. Сер. матем., 19:4 (1955), 187-200.

[34] Л. А. Дикий, "Формулы следов для дифференциальных операторов ШтурмаЛиувилля", УМН, 13:3 (1958), 111-143.

[35] Б. М. Левитан, "Вычисление регуляризованного следа для оператора ШтурмаЛиувилля", УМН, 19:1 (1964), 161-165.

[36] C. J. A. Halberg, Jr., V.A. Kramer, "A generalization of the trace concept", Duke Math. J., 27:4 (1960), 607-617.

[37] М. Г. Гасымов, Б. М. Левитан, "О сумме разностей собственных значений двух сингулярных операторов Штурма-Лиувилля”, Докл. АН СCCP, 151:5 (1963), 1014-1017.

[38] С. И. Гринберг, “Формула следов для уравнения Шредингера в конечной области", Матем. заметки, 1:4 (1967), 451-460.

[39] Р. Ф. Шевченко, "Регуляризация следа обыкновенного дифференциального оператора", Вестн. МГУ. Сер. 1. Матем., мех., 1965, № 6, 28-36.

[40] А.Г. Костюченко, О некоторых спектральных свойствах дифференииальных операторов, Дис. ... докт. физ.-матем. наук, МГУ, М., 1966.

[41] В. А. Садовничий, "О следе разности двух обыкновенных дифференциальных операторов высших порядков”, Дифферени. уравнения, 2:12 (1966), 1611-1624.

[42] В.А. Садовничий, "О следах обыкновенных дифференциальных операторов высших порядков", Матем. сб., 72:2 (1967), 293-317. 
[43] В. А. Садовничий, "О тождествах для собственных значений системы Дирака и некоторых других систем высшего порядка", Вестн. МГУ. Сер. 1. Матем., мех., 1967, № 3, 37-47.

[44] Э. Абдукадыров, "Вычисление регуляризованного следа для системы Дирака", Вестн. МГУ. Сер. 1. Матем., мех., 1967, № 4, 17-24.

[45] А. Зоммерфельд, Строение атома и спектры, т. 1, Гостехиздат, М., 1956.

[46] М.А. Наймарк, Линейные дифференииальные операторы, Наука, М., 1969.

[47] В. Б. Лидский, В. А. Садовничий, “Формулы следов в случае уравнения ОрраЗоммерфельда", Изв. АН СССР. Сер. матем., 32:3 (1968), 633-648.

[48] В.А. Садовничий, "Регуляризованные суммы полуцелых степеней оператора Штурма-Лиувилля", Матем. заметки, 14:2 (1973), 279-290.

[49] В. А. Садовничий, "О некоторых тождествах для собственных чисел сингулярных обыкновенных дифференциальных операторов. Соотношения для нулей функций Бесселя", Вестн. МГУ. Сер. 1. Матем., мех., 1971, № 3, 77-86.

[50] П. Л. Капица, "Вычисление сумм отрицательных четных степеней корней бесселевых функций”, Докл. АН СССР, 77:4 (1951), 561-564.

[51] Н. Н. Мейман, "О рекуррентных формулах для степенных сумм нулей бесселевых функций”, Докл. АН СССР, 108:2 (1956), 190-193.

[52] В. А. Садовничий, "О следах с весом и об асимптотике спектральной функции", Дифферени. уравнения, 10:10 (1974), 1808-1818.

[53] А.А. Стакун, "О свойствах оператора Шредингера с комплекснозначным потенциалом", Дифферени. уравнения, 32:3 (1996), 423-425.

[54] А. С. Печенцов, "Регуляризованные следы дифференциальных операторов: метод Лидского-Садовничего", Дифферени. уравнения, 35:4 (1999), 490-497.

[55] А. С. Печенцов, "Следы одного класса сингулярных дифференциальных операторов: метод Лидского-Садовничего", Вестн. МГУ. Сер. 1. Матем., мех., 1999, № $5,35-42$.

[56] Ю. Белаббаси, "Регуляризованные следы многоточечной задачи для обыкновенных дифференциальных операторов высших порядков", Дифферени. уравнения, 19:6 (1983), 938-944.

[57] М. Мартинович, “Дзета-функция и формулы следов для одной краевой задачи с функционально-дифференциальным уравнением", Дифферени. уравнения, 18:3 (1982), 537-540.

[58] 3. Каделбург, “Об асимптотике «спектральных функций» двух несамосопряженных краевых задач", Матем. заметки, 30:5 (1981), 659-667.

[59] 3. Каделбург, "Об асимптотике спектральной функции для обыкновенных дифференциальных операторов, заданных двучленной операцией на отрезке", Дифферени. уравнения, 18:9 (1982), 1475-1480.

[60] 3. Каделбург, М. Мартинович, "Спектральная функция одного функциональнодифференциального оператора второго порядка", Дифберени. уравнения, 25:11 (1989), 1882-1888.

[61] С.И. Митрохин, "О некоторых спектральных свойствах дифференциальных операторов второго порядка с разрывной положительной весовой функцией”, Докл. РАН, 356:1 (1997), 13-15.

[62] М. Пикула, "О регуляризованных следах дифференциального оператора типа Штурма-Лиувилля с запаздывающим аргументом", Дифферени. уравнения, 26:1 (1990), 103-109.

[63] В.А. Садовничий, В.А. Любишкин, Ю. Белаббаси, "О нулях целых функций одного класса", Тр. сем. им. И. Г. Петровского, 8 (1982), 211-217.

[64] В.А. Садовничий, В.А. Любишкин, "Регуляризованные суммы корней одного класса целых функций экспоненциального типа", Докл. АН СССР, 256:4 (1981), 794-798. 
[65] С. А. Салех, "Регуляризованные следы многоточечных задач", Вестн. МГУ. Сер. 1. Матем., мех., 1985, № 5, 64-66.

[66] Г. С. Типенко, "Регуляризованные суммы вычетов для одного класса целых функций экспоненциального типа", Вестн. МГУ. Сер. 1. Матем., мех., 1985, № 1, 93-96.

[67] Ю. В. Матвеев, "Некоторые свойства собственных функций дифференциальнофункционального оператора", Вестн. МГУ. Сер. вычисл. матем. и киберн., 1984, № 3, 54-59.

[68] В. С. Буслаев, "Формулы следов для оператора Шрёдингера в трехмерном пространстве”, Докл. АН СССР, 143:5 (1962), 1067-1070.

[69] В.С. Буслаев, “Формулы следов и некоторые асимптотические оценки ядра резольвенты для оператора Шрёдингера в трехмерном пространстве", Cneктральная теория и волновые прочессы, Проблемы матем. физики, 1, № 1, ред. М.Ш. Бирман, Изд-во ЛГУ, Л., 1966, 82-101.

[70] В. С. Буслаев, "Рассеянные плоские волны, спектральные асимптотики и формулы следа во внешних задачах", Докл. АН СCCP, 197:5 (1971), 999-1002.

[71] A. Rybkin, "On a trace formula of the Buslaev-Faddeev type for a long-range potential", J. Math. Phys., 40:3 (1999), 1334-1343.

[72] S. M. Belov, A. V. Rybkin, "Higher order trace formulas of the Buslaev-Faddeev-type for the half-line Schrödinger operator with long-range potential", J. Math. Phys., 44:7 (2003), 2748-2761.

[73] Ф. А. Березин, "Формула следов для многочастичного уравнения Шредингера", Докл. АН СССР, 157:5 (1964), 1069-1072.

[74] В. С. Буслаев, “Формула следа и сингулярности матрицы рассеяния для системы трех одномерных частиц. Третий групповой интеграл”, ТМФ, 16:2 (1973), 247259.

[75] С. П. Меркурьев, "Об $S$-матричной регуляризации формулы следа для системы трех частиц", Записки науч. сем. ЛОМИ, 63 (1976), 95-131.

[76] E. Beth, G. E. Uhlenbeck, "The quantum theory of the non-ideal gas. II: Behaviour at low temperatures", Physica, 4:10 (1937), 915-924.

[77] Д.Р. Яфаев, "О формуле следа в многоканальной модели Фридрихса", Краевые задачи математической физики, т. 10, Тр. МИАН, 147, ред. О. А. Ладыженская, Наука, Л., 1980, 194-201.

[78] С. А. Степин, "Спектр резонансов и формула следа в задаче потенциального рассеяния", Функи. анализ и его прилож., 38:3 (2004), 79-89.

[79] А. В. Рыбкин, "Формула следов для резонансов", ТМФ, 56:3 (1983), 439-447.

[80] H. Hochstadt, "On the determination of a Hill's equation from its spectrum", Arch. Rational Mech. Anal., 19:5 (1965), 353-362.

[81] И. М. Гельфанд, Л. А. Дикий, “Асимптотика резольвенты штурм-лиувиллевских уравнений и алгебра уравнений Кортевега-де Фриза", УМН, 30:5 (1975), 67-100.

[82] Б. М. Левитан, "Еще один способ вычисления плотностей интегралов движения для уравнения Кортевега-де Фриза", Матем. заметки, 22:1 (1977), 129-135.

[83] М. Абловиц, Х. Сигур, Солитоны и метод обратной задачи, Мир, М., 1987.

[84] F. Gesztesy, H. Holden, "Trace formulas and conservation laws for nonlinear evolution equations", Rev. Math. Phys., 6:1 (1994), 51-95.

[85] A. Rybkin, "KdV invariants and Herglotz functions", Differential Integral Equations, 14:4 (2001), 493-512.

[86] P. Deift, R. Killip, "On the absolutely continuous spectrum of one-dimensional Schrödinger operators with square summable potentials", Comm. Math. Phys., 203:2 (1999), 341-347.

[87] H. P. McKean, P. van Moerbeke, "The spectrum of Hill's equation", Invent. Math., 30:3 (1975), 217-274. 
[88] H. Flaschka, "On the inverse problem for Hill's operator", Arch. Rational Mech. Anal., 59:4 (1975), 293-309.

[89] S. Kotani, M. Krishna, "Almost periodicity of some random potentials", J. Funct. Anal., 78:2 (1988), 390-405.

[90] W. Craig, "The trace formula for Schrödinger operators on the line", Comm. Math. Phys., 126:2 (1989), 379-407.

[91] Б. М. Левитан, "Регуляризованные следы и условия гладкой периодичности потенциала уравнения Штурма-Лиувилля", Сиб. матем. журн., 22:2 (1981), 137148.

[92] Г. Ш. Гусейнов, Б. М. Левитан, "О формулах следов для операторов ШтурмаЛиувилля”, Вестн. МГУ. Сер. 1. Матем., мех., 1978, № 1, 40-49.

[93] В.А. Садовничий, В. А. Любишкин, "Конечномерные возмущения дискретных операторов и формулы следов", Функи. анализ и его прилож., 20:3 (1986), 55-65.

[94] В.В.Дубровский, А. С. Печенцов, "Регуляризованные следы эллиптических операторов высших порядков", Дифферени. уравнения, 29:1 (1993), 50-53.

[95] В. А. Любишкин, И. Д. Цопанов, “Регуляризованные следы интегро-дифференциальных операторов", Матем. заметки, 43:6 (1988), 786-793.

[96] В. В. Дубровский, "Формулы регуляризованных следов операторов с компактной резольвентой”, Дифферени. уравнения, 26:12 (1990), 2046-2051.

[97] В. В. Дубровский, “Абстрактные формулы регуляризованных следов эллиптических гладких дифференциальных операторов, заданных на компактных многообразиях", Дифферени. уравнения, 27:12 (1991), 2164-2166.

[98] В. В. Дубровский, Е. А. Пузанкова, “Оценка разности спектральных функций и формулы регуляризованных следов степени оператора Лапласа, заданного на треугольнике или квадрате, в $L_{p}, 1 \leqslant p \leqslant 2$ ", Дифферени. уравнения, 35:4 (1999), $552-555$.

[99] В. А. Любишкин, "О формулах следов Гельфанда-Левитана и Крейна", Матем. сб., 182:12 (1991), 1786-1795.

[100] В. В. Дубровский, А. И. Седов, “Оценка разности спектральных функций операторов типа Лежандра", Фундам. и прикл. матем., 6:4 (2000), 1075-1082.

[101] В. А. Садовничий, В.В.Дубровский, Н. Ю. Соченко, "Регуляризованные следы несамосопряженных дискретных операторов с неядерной резольвентой", Докл. PAH, 370:1 (2000), 24-26.

[102] А. Г. Баскаков, "Формулы регуляризованных следов для степеней возмущенных спектральных операторов", Изв. вузов. Сер. матем., 1985, № 8, 68-71.

[103] С. В. Сидоренко, “О формулах регуляризованных следов", УМН, 54:5 (1999), $173-174$.

[104] M. Dostanić, "Trace formulas of Gel'fand-Levitan type", Publ. Inst. Math. (Beograd) (N.S.), 55(69) (1994), 51-63.

[105] В.А. Садовничий, В.Е. Подольский, "Регуляризованный след ограниченного возмущения оператора с ядерной резольвентой”, Дифферени. уравнения, 35:4 (1999), 556-564.

[106] В.А. Садовничий, С. В. Конягин, В.Е. Подольский, "Регуляризованный след оператора с ядерной резольвентой, возмущенного ограниченным", Докл. РАН, 373:1 (2000), 26-28.

[107] В. В. Дубровский, А. Н. Типко, З. С. Чекашкина, "Регуляризованные следы унитарных операторов", УМН, 56:6 (2001), 145-146.

[108] Х. Х. Муртазин, З. Ю. Фазуллин, "О формулах следов для неядерных возмущений", Докл. РАН, 368:4 (1999), 442-444.

[109] Х. Х. Муртазин, З. Ю. Фазуллин, "Неядерные возмущения дискретных операторов и формулы следов", Матем. сб., 196:12 (2005), 123-156. 
[110] Т. Г. Амангильдин, "Регуляризованный след оператора Штурма-Лиувилля", Дифферени. уравнения, 25:8 (1989), 1439-1441.

[111] Г. В. Козлов, В. А. Любишкин, "Регуляризованные следы сингулярных дифференциальных операторов", Вестн. МГУ. Сер. 1. Матем., мех., 1993, № 4, 6-11.

[112] Г. В. Козлов, В.А.Любишкин, "Регуляризованные следы высших порядков для гармонического осциллятора", Дифферени. уравнения, 29:1 (1993), 61-63.

[113] А. И. Козко, А. С. Печенцов, "Спектральная функция и регуляризованные следы сингулярных дифференциальных операторов высших порядков”, Докл. РАН, 401:2 (2005), 160-162.

[114] В.А. Любишкин, "Регуляризованные следы оператора Штурма-Лиувилля на полуоси в случае неограниченно убывающего потенциала", Дифберени. уравнения, 18:2 (1982), 345-346.

[115] В. В. Дубровский, А. И. Седов, “Асимптотика собственных значений сингулярного дифференциального оператора типа Якоби”, Докл. РАН, 353:3 (1997), 295299.

[116] В. Е. Подольский, "О восстановлении оператора Штурма-Лиувилля по его весовой $\zeta$-функции", Докл. АН СССР, 313:3 (1990), 559-562.

[117] Е. В. Александрова, “Формула следа для уравнений колебаний прямолинейного трубопровода", Дифферени. уравнения, 33:1 (1997), 12-18.

[118] В. Б. Лидский, "О суммируемости рядов по главным векторам несамосопряженных операторов", Тр. МMO, 11 (1962), 3-35.

[119] В. Е. Подольский, “Суммирование по Абелю регуляризованных следов”, Вестн. МГУ. Сер. 1. Матем., мех., 1999, № 5, 42-48.

[120] Е. М. Сантанжело, "Вычисление энергии Казимира с помощью спектральных функций", ТМФ, 131:1 (2002), 98-117.

[121] E. Elizalde, "Zeta functions: formulas and applications", J. Comput. Appl. Math., 118:1-2 (2000), 125-142.

[122] Е. В. Александрова, О.В.Бочкарева, В. Е. Подольский, "Суммирование регуляризованных следов сингулярного оператора Штурма-Лиувилля", Дифферени. уравнения, 33:3 (1997), 291-295.

[123] Е. В. Александрова, Формула следа гармонического осииллятора с нефинитным возмущением, Деп. в ВИНИТИ РАН 18.01.1997 № 129-В97.

[124] A. Pushnitski, I. Sorrell, "High energy asymptotics and trace formulas for the perturbed harmonic oscillator", Ann. Henri Poincaré, 7:2 (2006), 381-396.

[125] M. Hitrik, I. Polterovich, "Regularized traces and Taylor expansions for the heat semigroup", J. London Math. Soc. (2), 68:2 (2003), 402-418.

[126] В. Е. Подольский, "О следе возмущенной операторной полугруппы", Maтем. заметки, 75:3 (2004), 462-465.

[127] Н. Харт, Геометрическое квантование в действии: Приложения гармонического анализа в квантовой статистической механике и квантовой теории поля, Мир, М., 1985.

[128] В.В.Дубровский, "О регуляризованных следах дифференциальных операторов в частных производных", Тр. сем. им. И. Г. Петровского, 9 (1983), 40-44.

[129] А. Н. Бобров, В. Е. Подольский, "Сходимость регуляризованных следов степени оператора Лапласа-Бельтрами с потенциалом на сфере $S^{n}$ ”, Матем. сб., 190:10 (1999), 3-16.

[130] А.Н. Бобров, "Регуляризованные следы высших порядков оператора Лапласа с потенциалом на симметрических пространствах ранга 1", Дифферени. уравнения, 33:6 (1997), 800-804.

[131] В. А. Садовничий, З. Ю. Фазуллин, “Асимптотика собственных чисел и формула следа возмущения оператора Лапласа на сфере $S^{2}$ ", Матем. заметки, 77:3 (2005), 434-448. 
[132] В.А. Любишкин, "Регуляризованный след двумерного осциллятора", Матем. заметки, 53:3 (1993), 156-158.

[133] З. Ю. Фазуллин, Х.Х. Муртазин, "Классическая формула регуляризованного следа многомерного гармонического осциллятора", Тр. сем. им. И.Г. Петровского, 21 (2001), 298-339.

[134] Х.Х. Муртазин, З. Ю. Фазуллин, "Спектр и формула следов для двумерного оператора Шредингера в однородном магнитном поле”, Докл. РАН, 390:6 (2003), $743-745$.

[135] E. Korotyaev, A. Pushnitski, "A trace formula and high-energy spectral asymptotics for the perturbed Landau Hamiltonian", J. Funct. Anal., 217:1 (2004), 221-248.

[136] M. R. Dostanić, "Spectral properties of the operator of Riesz potential type", Proc. Amer. Math. Soc., 126:8 (1998), 2291-2297.

[137] Ю. В. Коган, “Формулы следов для псевдодифференциальных операторов на окружности", Вестн. МГУ. Сер. 1. Матем., мех., 1980, №6, 7-11.

[138] В. Е. Подольский, "Регуляризованные следы некоторых псевдодифференциальных операторов на окружности", Вестн. МГУ. Сер. 1. Матем., мех., 1988, №6, $11-14$.

[139] А.Н. Бобров, "Спектральные свойства некоторых двумерных краевых задач", Вестн. МГУ. Сер. 1. Матем., мех., 2001, №1, 3-6.

[140] И. М. Лифшиц, "О вырожденных регулярных возмущениях. I: Дискретный спектр", ЖЭТФ, 17:11 (1947), 1017-1025.

[141] И. М. Лифшиц, "О вырожденных регулярных возмущениях. II: Квазинепрерывный и непрерывный спектры”, ЖЭТФ, 17:12 (1947), 1076-1089.

[142] М. Г. Крейн, “Об определителях возмущения и формуле следов для унитарных и самосопряженных операторов”, Докл. АН СССР, 144:2 (1962), 268-271.

[143] М.Ш. Бирман, М. Г. Крейн, "К теории волновых операторов и операторов рассеяния", Докл. АН СССР, 144:3 (1962), 475-478.

[144] В.А. Яврян, "О функции спектрального сдвига для операторов ШтурмаЛиувилля", Докл. АН АрмССР, 38:4 (1964), 193-198.

[145] Л. С. Коплиенко, “О формуле следов для возмущений неядерного типа", Сиб. матем. журн., 25:5 (1984), 62-71.

[146] H. Neidhardt, "Spectral shift function and Hilbert-Schmidt perturbation: extensions of some work of L. S. Koplienko", Math. Nachr., 138 (1988), 7-25.

[147] M. Dostanić, "Trace formula for nonnuclear perturbations of selfadjoint operators", Publ. Inst. Math. (Beograd) (N.S.), 54(68) (1993), 71-79.

[148] А.В. Рыбкин, "Функция спектрального сдвига, характеристическая функция сжатия и обобщенный интеграл", Матем. сб., 185:10 (1994), 91-144.

[149] А. Полторацкий, "Спектральный сдвиг Крейна и возмущения спектров ранга один", Алгебра и анализ, 10:5 (1998), 143-183.

[150] А. Б. Пушницкий, "Представление для функции спектрального сдвига в случае знакоопределенных возмущений”, Алгебра и анализ, 9:6 (1997), 197-213.

[151] А.Б. Пушницкий, "Интегральные оценки для функции спектрального сдвига", Алгебра и анализ, 10:6 (1998), 198-233.

[152] E. Korotyaev, A. Pushnitski, "Trace formulae and high energy asymptotics for the Stark operator", Comm. Partial Differential Equations, 28:3-4 (2003), 817-842.

[153] W. Müller, "Relative zeta functions, relative determinants and scattering theory", Comm. Math. Phys., 192:2 (1998), 309-347.

[154] F. Gesztesy, B. Simon, "The xi function", Acta Math., 176:1 (1996), 49-71.

[155] A. Rybkin, "Necessary and sufficient conditions for absolute summability of the trace formulas for certain one dimensional Schrödinger operators", Proc. Amer. Math. Soc., 131:1 (2003), 219-229. 
[156] В. А. Садовничий, В.Е. Подольский, "Об обобщенной функции спектрального сдвига и связи формул следа Крейна и Гельфанда-Левитана", Докл. РАН, 402:3 (2005), 311-312.

[157] H. Weyl, "Ramifications, old and new, of the eigenvalue problem", Bull. Amer. Math. Soc., 56 (1950), 115-139.

[158] Р. Т. Сили, “Степени эллиптического оператора", Математика, 12:1 (1968), 96112.

[159] М. А. Шубин, Псевдодифференииальные операторы и спектралъная теория, Наука, М., 1978.

[160] M. F. Atiyah, V.K. Patodi, I. M. Singer, "Spectral asymmetry and Riemannian geometry, III", Math. Proc. Cambridge Philos. Soc., 79:1 (1976), 71-99.

[161] P. B. Gilkey, "The residue of the global $\eta$-function at the origin", Advin. in Math., 40:3 (1981), 290-307.

[162] M. Wodzicki, "Noncommutative residue. I: Fundamentals", K-theory, aritmetic and geometry (Moscow, 1984-1986), Lecture Notes in Math., 1289, Springer, Berlin, 1987, 320-399.

[163] В.А. Садовничий, “Дзета-функция и собственные числа дифференциальных операторов", Дифферени. уравнения, 10:7 (1974), 1276-1285.

[164] G. Illies, "Regularized products and determinants", Comm. Math. Phys., 220:1 (2001), 69-94.

[165] Б. В. Федосов, “Аналитические формулы индекса эллиптических операторов", Tp. MMO, 30 (1974), 159-241.

[166] Б. В. Федосов, "Об индексе эллиптических семейств на многообразии с краем", Докл. АН СССР, 248:5 (1979), 1066-1069.

[167] Б. В. Федосов, М. А. Шубин, "Индекс случайных эллиптических операторов. II", Матем. сб., 106(148):3 (1978), 455-483.

[168] Ш. Ремпель, Б.-В. Шульце, Теория индекса эллиптических краевых задач, Мир, M., 1986.

[169] D. Bollé, F. Gesztesy, H. Grosse, W. Schweiger, B. Simon, "Witten index, axial anomaly, and Krein's spectral shift function in supersymmetric quantum mechanics", J. Math. Phys., 28:7 (1987), 1512-1525.

[170] F. Gesztesy, B. Simon, "Topological invariance of the Witten index", J. Funct. Anal., 79:1 (1988), 91-102.

[171] Л. А. Дикий, "Новый способ приближенного вычисления собственных чисел задачи Штурма-Лиувилля”, Докл. АН СССР, 116:1 (1957), 12-14.

[172] А. А. Дородницын, "Асимптотические законы распределения собственных значений для некоторых особых видов дифференциальных уравнений второго порядка", УМН, 7:6 (1952), 3-96.

[173] С. А. Шкарин, "О способе Гельфанда-Дикого вычисления первых собственных значений оператора Штурма-Лиувилля", Вестн. МГУ. Сер. 1. Матем., мех., 1996, № 1, 39-44.

[174] В. А. Садовничий, В. Е. Подольский, "О неединственности решения системы регуляризованных следов", Докл. РАН, 402:4 (2005), 455-456.

[175] В.А. Садовничий, В.Е. Подольский, "Об одном классе операторов ШтурмаЛиувилля и приближенном вычислении первых собственных значений”, Матем. сб., 189:1 (1998), 133-148.

[176] В.А.Садовничий, В.В.Дубровский, Е. М. Малеко, “Об одном способе приближенного нахождения собственных чисел оператора Штурма-Лиувилля”, Докл. РАН, 369:1 (1999), 16-18.

[177] В. А. Садовничий, В.В.Дубровский, “Замечания об одном новом методе вычисления собственных значений и собственных функций дискретного оператора", Тр. сем. им. И. Г. Петровского, 17 (1994), 244-248. 
[178] В.А.Садовничий, В.В.Дубровский, С. И. Кадченко, В.Ф. Кравченко, "Вычисление первых собственных чисел краевой задачи гидродинамической устойчивости течения Пуазейля в круглой трубе", Дифферени. уравнения, 34:1 (1998), $50-53$.

[179] В.А.Садовничий, В.В.Дубровский, С. И. Кадченко, В. Ф. Кравченко, "Вычисление первых собственных чисел задачи гидродинамической устойчивости течения вязкой жидкости между двумя вращающимися цилиндрами", Дифферени. уравнения, 36:6 (2000), 742-746.

[180] P. Nevai, "Orthogonal polynomials, recurrences, Jacobi matrices, and measures", Progress in Approximation Theory (Tampa, FL, 1990), Springer Ser. Comput. Math., 19, Springer, New York, 1992, 79-104.

[181] Б. П. Осиленкер, “Аналог формулы следа для дискретных операторов ШтурмаЛиувилля с асимптотически $N$-периодическими коэффициентами”, Функи. анализ и его прилож., 31:4 (1997), 72-75.

[182] R. Killip, B. Simon, "Sum rules for Jacobi matrices and their applications to spectral theory", Ann. of Math. (2), 158:1 (2003), 253-321.

[183] Р.Ш. Бакурадзе, И. В. Станкевич, "О некоторых тождествах для собственных чисел и собственных функций одного класса дифференциальных операторов четвертого порядка", Дифферени. уравнения, 26:9 (1990), 1483-1490.

\section{В. А. Садовничий (V. A. Sadovnichii)}

Московский государственный университет им. М. В. Ломоносова

\section{В. Е. Подольский (V. E. Podol'skii)}

Московский государственный университет

им. М. В. Ломоносова

E-mail: wpve@yandex.ru
Поступила в редакцию 23.04.2006 